\title{
Tangles, trees, and flowers
}

by

Ben Clark

A thesis

submitted to the Victoria University of Wellington

in fulfilment of the

requirements for the degree of

Master of Science

in Mathematics.

Victoria University of Wellington

2011 


\begin{abstract}
A tangle of order $k$ in a connectivity function $\lambda$ may be thought of as a " $k$-connected component" of $\lambda$. For a connectivity function $\lambda$ and a tangle in $\lambda$ of order $k$ that satisfies a certain robustness condition, we describe a tree decomposition of $\lambda$ that displays, up to a certain natural equivalence, all of the $k$-separations of $\lambda$ that are non-trivial with respect to the tangle. In particular, for a tangle in a matroid or graph of order $k$ that satisfies a certain robustness condition, we describe a tree decomposition of the matroid or graph that displays, up to a certain natural equivalence, all of the $k$ separations of the matroid or graph that are non-trivial with respect to the tangle.
\end{abstract}




\section{Acknowledgements}

I wish to thank all those who have helped and supported me during my studies. In particular, I wish to thank my supervisor, Geoff Whittle, for the enthusiasm and generous support that he provided throughout this thesis. 


\section{Contents}

$\begin{array}{lll}1 & \text { Introduction } & 1\end{array}$

2 Preliminaries $\mathbf{5}$

2.1 Connectivity . . . . . . . . . . . . . . . . . . . 5

$2.1 .1 \quad$ Connectivity systems . . . . . . . . . . . . . 5

$2.1 .2 \quad$ Matroid and graph connectivity . . . . . . . . . 7

2.2 Tree-width, branch-width, and tangles $\ldots \ldots \ldots . . \ldots 8$

2.2 .1 Tree-width and branch-width . . . . . . . . . . . . 9

2.2 .2 Tangles $\ldots \ldots \ldots \ldots$

2.2 .3 Tangles of order three in connected matroids . . . . . . 15

2.3 The 3-separations of 3-connected matroids . . . . . . . . . . . 18

2.3 .1 Sequential and equivalent 3-separations . . . . . . . . . 19

2.3 .2 Flowers . . . . . . . . . . . . . . . . . . . 20

2.3 .3 Partial 3-trees . . . . . . . . . . . . . . . . . . 24

2.4 Higher-order separations . . . . . . . . . . . . . . 26

$2.4 .1 \quad k$-flowers . . . . . . . . . . . . . . . . . . 27

2.4 .2 The 4-separations of 4-connected matroids . . . . . . . 28

$\begin{array}{lll}3 & \text { Flowers } & 29\end{array}$

3.1 Connectivity systems $\ldots \ldots \ldots$

3.2 Tangles . . . . . . . . . . . . . . . . . . . . . . . . . . . . . 31

3.3 Sequential and equivalent $k$-separations . . . . . . . . . . . . . 32

3.4 Flowers in a tangle . . . . . . . . . . . . . . . . . . . . . . 40 
3.5 Conformity . . . . . . . . . . . . . . . . . . 47

3.6 Robustness $\ldots \ldots \ldots \ldots$. . . . . . . . . . . . . . . . . . . . . 54

3.7 Accumulating connectivity . . . . . . . . . . . . . . . . 57

4 Tree decomposition $\quad 62$

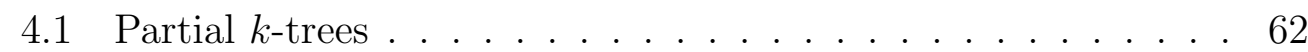

4.2 Proof of the main theorem . . . . . . . . . . . . . . . 71

$\begin{array}{lll}5 & \text { Applications } & 77\end{array}$

$5.1 \quad$ Tangles in $k$-connected matroids . . . . . . . . . . . . . . . . 77

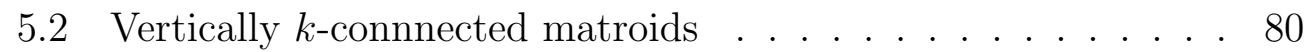

\begin{tabular}{ll}
\hline Bibliography & 83
\end{tabular}

\begin{tabular}{lr}
\hline Index & 87
\end{tabular} 


\section{Chapter 1}

\section{Introduction}

Robertson and Seymour's Graph-Minors Project contains a number of deep theorems on the structure of graphs, including the celebrated result that finite graphs are well-quasi-ordered under the minor relation [30]. A crucial concept in the Graph-Minors Project is that of a tangle in a graph, which was introduced in [28]. Intuitively, a tangle of order $k$ in a graph $G$ may be thought of as a " $k$-connected component" of $G$, so that most of the tangle lies on one side or the other of each separation of order less than $k$.

Tangles have a natural extension to matroids, which was first noted by Robertson and Seymour [28]. For matroids, tangles play an important role in structure theory. Indeed, tangles are central to Geelen, Gerards, and Whittle's on-going research aimed at extending the work of the Graph-Minors Project to matroids [10, 12]. Broadly speaking, tangles are used to get a handle on the highly-connected regions of a matroid.

Historically, a useful tool for understanding the structure of a matroid has been a tree that describes how a matroid may be decomposed into smaller, more easily understood pieces. Cunningham and Edmonds [4] proved that every connected matroid has an associated tree that shows precisely how the matroid is decomposed into 3-connected pieces. More recently, in a study of the structure of the 3-separations of 3-connected matroids, Oxley, Semple, and Whittle [23] described a tree decomposition of a 3-connected matroid 
$M$ that displays, up to a natural equivalence, all of the "non-trivial" 3separations of $M$. Understanding the structure of the 3-separations of 3connected matroids has opened the way for substantial progress to be made. For example, Geelen and Whittle [14] have proved that 3-connected matroids with suitably controlled 3-separations have a bounded number of inequivalent representations over prime fields.

The success of Oxley, Semple, and Whittle [23] in describing the structure of the 3 -separations of 3 -connected matroids has provided motivation to pursue an analogous theory for higher-order separations, and progress has already been made towards this goal. Specifically, Aikin and Oxley [2] have completely classified the types of crossing separations that can arise in arbitrary matroids. Though we have not seen their work, Aikin and Oxley (private communication) have also described a tree decomposition of a 4connected matroid $M$ that displays, up to a natural equivalence, all of the "non-trivial" 4-separations of $M$.

The work of Aikin and Oxley encourages the belief that an analogous theory for higher-order separations is attainable. However, further progress is complicated by the fact that high connectivity is generally too restrictive. For example, projective geometries and the cycle matroids of complete graphs are generally not highly-connected. Thus, a matroid of interest may have several highly-connected parts that are separated by low-order separations. An approach that studies the separations of a matroid relative to its tangles could overcome this, because the tangles of a matroid identify its highlyconnected parts very successfully. This motivates a study of the separations of a matroid with respect to its tangles, and this thesis is the outcome.

Before discussing the main result and the structure of this thesis in more detail, we note two important features. First, we note that though we had a theory for matroids in mind, the theory we developed relies only on the fact that the connectivity function of a matroid is an integer-valued, symmetric, and submodular function. The theory stated for arbitrary connectivity functions undoubtedly has the potential for broader application, so we chose to 
present it for such functions. Second, of the works we have mentioned, the one by Oxley, Semple, and Whittle [23] was particularly influential in the development of this thesis. A 3-connected matroid has a unique tangle of order three, and via this connection the theory presented here extends parts of their theory. But many of the definitions and concepts introduced in this thesis are essentially those of Oxley, Semple, and Whittle. The statements of Theorem 3.6.1 and Theorem 4.2.1 are also modelled closely after their work.

Loosely speaking, the main result of this thesis, Theorem 4.2.1, says that, for a connectivity function $\lambda$ and a tangle in $\lambda$ of order $k$ that satisfies a certain robustness condition, there is a tree decompostion of $\lambda$ that displays, up to a certain natural equivalence, all of the $k$-separations of $\lambda$ that are nontrivial with respect to the tangle. In particular, for a 3-connected matroid $M$ with at least nine elements, the unique tangle of order three in $M$ satisfies the robustness condition, so Theorem 4.2.1 extends the main result of Oxley, Semple, and Whittle [23].

We now discuss the structure of this thesis in more detail. Chapter 2 introduces some background material on connectivity, width parameters and tangles, and the structure of crossing separations in matroids.

Chapter 3 is the first main chapter. We start by recalling connectivity systems and tangles, and we establish some notions for these structures that will be used frequently. We then define a suitable notion of equivalence on the $k$-separations of a connectivity system with respect to a fixed tangle of order $k$, and also define what it means for a $k$-separation of a connectivity system to be sequential with respect to a fixed tangle of order $k$. Following Oxley, Semple, and Whittle [23], we make no attempt to display the sequential $k$ separations, and we only attempt to display the non-sequential $k$-separations up to equivalence. We introduce the notion of a $k$-flower in a tangle of order $k$ in Section 3.4 to study collections of crossing $k$-separations. There is a natural quasi-order on $k$-flowers induced by the non-sequential $k$-separations they display. In Section 3.5, we study how the non-sequential $k$-separations interact with $k$-flowers, and we find conditions under which we can refine 
a $k$-flower. Theorem 3.6.1, the main result of Chapter 3 , shows that if we introduce a notion of robustness for tangles, then all of the non-sequential $k$-separations interact with a maximal $k$-flower in a coherent way. Theorem 3.6.1 plays an essential role in obtaining the tree structure described in Theorem 4.2.1. We conclude with a section of independent interest in which we obtain a bound on the number of petals in a sequential $k$-separation displayed by a tight $k$-flower.

Chapter 4 is the second main chapter. Here we introduce the notion of a partial $k$-tree for a tangle of order $k$ in a connectivity system, which is a tree that we can associate with the tangle, some of whose vertices are labelled by members of a partition of the underlying set. Partial $k$-trees display $k$ separations, so just as with $k$-flowers there is a quasi-order on partial $k$-trees induced by the non-sequential $k$-separations they display. Theorem 4.2.1, the main result of Chapter 4 , then shows that a maximal partial $k$-tree displays, up to equivalence of $k$-separations, all of the non-sequential $k$-separations of a connectivity system with respect to a robust tangle of order $k$.

Finally, in Chapter 5 we conclude with some applications of the main results obtained in Chapters 3 and 4 to some particularly interesting structures, namely $k$-connected matroids and vertically $k$-connected matroids.

Here we make a note which results are pre-existing, and which are new. All of the results in Chapter 2 are pre-existing. Though the tangle-theoretic approach to the 2-sum decomposition in Section 2.2.3 is well-known, we are unaware of any presentation in the literature. Chapter 3 and Chapter 4 contain new material, with minor exceptions noted as they appear. The results of Chapter 5 are derived from earlier chapters. We note, however, that for 3-connected and 4-connected matroids, Corollary 5.1.4 of Section 5.1 is already known. 


\section{Chapter 2}

\section{Preliminaries}

In this chapter we review some fundamental background material. We assume that the reader is familiar with the basic concepts of matroid theory and graph theory. Any undefined notation or terminology will follow Oxley [22].

We fix some notation for certain subsets of positive integers that we use frequently. For any positive integer $n$, we let $[n]=\{1,2, \ldots, n\}$, and for integers $i \leq j$ we let $[i, j]=\{i, i+1, \ldots, j\}$.

Let $n$ be a positive integer. A partition of a set $S$ is a set $\left\{S_{1}, \ldots, S_{n}\right\}$ of subsets of $S$ such that each element of $S$ belongs to exactly one of the subsets $S_{1}, \ldots, S_{n}$. Thus we allow partitions to contain empty members.

\subsection{Connectivity}

\subsubsection{Connectivity systems}

Let $\lambda$ be an integer-valued function on the subsets of a finite set $E$. We call $\lambda$ symmetric if $\lambda(X)=\lambda(E-X)$ for all $X \subseteq E$. We call $\lambda$ submodular if $\lambda(X)+\lambda(Y) \geq \lambda(X \cup Y)+\lambda(X \cap Y)$ for all $X, Y \subseteq E$. If $\lambda$ is integer-valued, symmetric, and submodular, then $\lambda$ is called a connectivity function on E. If $E$ is a finite set and $\lambda$ is a connectivity function on $E$, then the pair $(E, \lambda)$ 
is a connectivity system.

We now introduce some examples of connectivity systems that arise naturally from combinatorial structures.

Example 1. Let $M$ be a matroid with rank function $r$. For all $X \subseteq E(M)$, we let $\lambda_{M}(X)=r(X)+r(E(M)-X)-r(M)+1$. It is straightforward to prove that $\left(E(M), \lambda_{M}\right)$ is a connectivity system. We call $\lambda_{M}$ the connectivity function of $M$. We note that the connectivity function of a matroid $M$ is invariant under duality, that is, $\lambda_{M}=\lambda_{M^{*}}$.

Example 2. A polymatroid on a set $E$ is an integer-valued, increasing, submodular function on the subsets of $E$ whose value on $\emptyset$ is 0 . We can generalise the construction of Example 1 to a polymatroid $f$ on $E$ as follows. For all $X \subseteq E$, we let $\lambda_{f}(X)=f(X)+f(E-X)-f(E)+1$. It is also straightforward to prove that $\left(E, \lambda_{f}\right)$ is a connectivity system.

Example 3. Let $G$ be a graph with edge set $E$. For $X \subseteq E$, we let $\lambda_{G}(X)$ denote the number of vertices of $G$ that are incident with both an edge of $X$ and an edge of $E-X$. It is not difficult to prove that $\left(E, \lambda_{G}\right)$ is a connectivity system. We call $\lambda_{G}$ the connectivity function of $G$. We also note that $\lambda_{M(G)}(X)=\lambda_{G}(X)+c(E)-c(X)-c(E-X)+1$, where $c(X)$ denotes the number of connected components of the induced subgraph on $X$.

Example 4. We can generalise the construction of Example 3 to a hypergraph $G$ on $E$ as follows. For $X \subseteq E$, we let $\lambda_{G}(X)$ denote the number of vertices of $G$ that are incident with both an edge of $X$ and an edge of $E-X$. Then $\left(E, \lambda_{G}\right)$ is a connectivity system.

Example 5. Let $G$ be a graph and let $X \subseteq V(G)$. Let $\lambda_{V(G)}(X)$ denote the number of edges incident with both a vertex in $X$ and a vertex in $V(G)-X$. Clearly $\lambda_{V(G)}$ is symmetric, and a simple counting argument shows that $\lambda$ is submodular, so $\left(V(G), \lambda_{V(G)}\right)$ is a connectivity system.

Example 6. We can generalise the construction of Example 5 to an edgeweighted graph $G$ as follows. Let $w: E(G) \longrightarrow \mathbb{Z}^{+} \cup\{0\}$ be a weighting 
of the edges of $G$, and let $X \subseteq V(G)$. We let $\lambda_{w}(X)$ denote the weight of the set of edges that are incident with both a vertex in $X$ and a vertex in $V(G)-X$. Then $\left(V(G), \lambda_{w}\right)$ is a connectivity system.

Let $\lambda$ be a connectivity function on $E$, and let $k$ be a positive integer. A partition $(X, E-X)$ of $E$ is called a $k$-separation of $\lambda$ if $\lambda(X) \leq k$. A subset $X$ of $E$ is said to be $k$-separating in $\lambda$ if $\lambda(X) \leq k$. When the connectivity function $\lambda$ is clear from the context we shall often abbreviate " $k$-separation of $\lambda$ " and " $k$-separating in $\lambda$ " to " $k$-separation" and " $k$-separating" respectively. A $k$-separating set $X$, or $k$-separation $(X, E-X)$ is exact if $\lambda(X)=k$.

There are two features of these definitions that are worth noting. First, we will follow the convention of matroid theory and consider a $k$-separation $(X, E-X)$ to be an unordered partition. Second, if $\lambda_{M}$ is the connectivity function of a matroid $M$, then a $k$-separation $(X, E(M)-X)$ of $\lambda_{M}$ is sometimes called a $k$-separating partition of $M$, while the term " $k$-separation" is reserved for those $k$-separating partitions $(X, E(M)-X)$ of $M$ that satisfy $\min \{|X|,|E(M)-X|\} \geq k$ (see, for example, [23]). In this thesis, we will only make such a distinction in the context of $k$-connected matroids.

\subsubsection{Matroid and graph connectivity}

We will later specialise results obtained for connectivity functions to matroids that satisfy certain connectivity conditions, so we introduce the necessary connectivity terminology here.

Let $M$ be a matroid with ground set $E$ and rank function $r$. Recall that the connectivity function $\lambda_{M}$ of $M$ is defined for all subsets $X$ of $E$ by $\lambda_{M}(X)=r(X)+r(E-X)-r(M)+1$. Let $k$ be a positive integer. The set $X$ or the partition $(X, E-X)$ is $k$-separating if $\lambda(X) \leq k$. A $k$-separating partition $(X, E-X)$ is a $k$-separation if $\min \{|X|,|E-X|\} \geq k$. A $k$ separating set, $k$-separating partition $(X, E-X)$, or $k$-separation $(X, E-X)$ is exact if $\lambda(X)=k$. For $k \geq 2$, a matroid $M$ is $k$-connected if it has no $j$-separations for $1 \leq j \leq k-1$. 
Let $G$ be a graph. If $G$ is a connected graph that has at least one pair of distinct non-adjacent vertices, then we define the connectivity $\kappa(G)$ of $G$ to be the smallest positive integer $k$ such that $G$ has a $k$-element vertex cut. If $G$ is connected but has no pair of non-adjacent vertices, then we let $\kappa(G)=|V(G)|-1$. Finally, if $G$ is disconnected, then we let $\kappa(G)=0$. For positive integer $k$, the graph $G$ is said to be $k$-connected if $\kappa(G) \geq k$.

We now describe a notion of matroid connectivity that directly generalises graph connectivity. Let $k$ be a positive integer. A matroid $M$ is vertically $k$-separated if there is a $k$-separating partition $(X, Y)$ of $E(M)$ such that $\min \{r(X), r(Y)\} \geq k$. If $M$ has a pair of disjoint cocircuits, then the vertical connectivity of $M$, denoted $\kappa(M)$, is the least positive integer $k$ such that $M$ is vertically $k$-separated; otherwise we let $\kappa(M)=r(M)$.

For a connected graph $G$, the following theorem makes precise the claim that vertical connectivity directly generalises graph connectivity.

Theorem 2.1.1. [22, Theorem 8.2.5] Let $G$ be a connected graph. Then

$$
\kappa(M(G))=\kappa(G)
$$

A matroid $M$ is cyclically $k$-connected if $M^{*}$ is vertically $k$-connected.

\subsection{Tree-width, branch-width, and tangles}

In this section we describe two important notions of graph "width", namely tree-width and branch-width, and their extensions to matroids. We discuss some of their important properties, including the connection between treewidth and branch-width. Although tree-width and branch-width will not appear later in this thesis, an understanding of these concepts motivates the study of tangles. In Section 2.2.2, we discuss the canonical obstructions to small branch-width, called tangles. Finally, to help the reader's intuition for tangles, we present a tangle-theoretic approach to the 2-sum decomposition for connected matroids. 


\subsubsection{Tree-width and branch-width}

In their ground-breaking work on graph minors, Robertson and Seymour proved that finite graphs are well-quasi-ordered under the minor relation [30]. In other words, in any infinite set of finite graphs, there is one that is isomorphic to a minor of another. The result is widely considered to be the deepest in all of graph theory, and many concepts developed in the proof are of independent interest. This is particularly true of tree-decompositions and the associated measures of tree-likeness.

We first discuss tree-width, which was introduced by Robertson and Seymour in [25]. Loosely speaking, a graph has small tree-width if it can be constructed by piecing together small graphs in a tree-like way. We make this notion precise by considering the following representation of a graph as a tree.

Let $G$ be a graph, $T$ a tree, and $\mathcal{V}=\left\{V_{t} \mid t \in V(T)\right\}$ a collection of vertex sets $V_{t} \subseteq V(G)$ indexed by the vertices of $T$. The pair $(T, \mathcal{V})$ is a tree-decomposition of $G$ if the following properties hold:

(i) $V(G)=\bigcup_{t \in V(T)} V_{t}$;

(ii) for every edge $e$ of $G$ there exists a $t \in V(T)$ such that both ends of $e$ are in $V_{t}$; and

(iii) for $s, t, u \in V(T)$, if $t$ lies on the path of $T$ from $s$ to $u$ then $V_{s} \cap V_{u} \subseteq V_{t}$.

Observe that conditions (i) and (ii) together show that $G$ is the union of the subgraphs $G\left[V_{t}\right]$, while (iii), roughly speaking, shows that these subgraphs are put together like a tree. The width of a tree-decomposition $(T, \mathcal{V})$ of $G$ is the number $\max \left\{\left|V_{t}\right|-1 \mid t \in V(T)\right\}$, and the tree-width of $G$, denoted tree-width $(G)$, is the smallest width of any tree-decomposition of $G$.

As one easily checks, the connected graphs of tree-width one are precisely the trees. Due to the simple structure of trees, many graph problems become easier when specialised to trees. A crucial feature of tree-width is that graphs of bounded tree-width are sufficiently similar to trees that it is often possible 
to adapt results for trees to this class of graphs. As an example of this paradigm, consider the problem of well-quasi-ordering graphs. Kruskal [20] proved that finite trees are well-quasi-ordered under the minor relation, and Nash-Williams [21] later gave an elegant proof of this fact using a 'minimal bad sequence' argument. Although their argument is substantially more difficult, Robertson and Seymour [27] adapted Nash-Williams' argument to prove that, for every positive integer $n$, every infinite set of graphs with treewidth at most $n$ is well-quasi-ordered under the minor relation. Robertson and Seymour's proof that finite graphs are well-quasi-ordered relies heavily on this result.

In addition to its importance in graph structure theory, tree-width also has significant algorithmic consequences. Indeed, many algorithmic problems that are known to be NP-hard for general graphs are solvable in polynomial time for inputs of bounded tree-width (see, for example, [3]).

Given the success of tree-width for graphs, it is natural to ask if the concept can be generalised to matroids. Although it is not clear from Robertson and Seymour's definition, tree-width can be defined without reference to graph vertices, and hence it extends to matroids (see [19]). However, a closely-related parameter, branch-width, has more natural properties for matroids, and has gained considerably more attention from matroid theorists.

Robertson and Seymour introduced the notion of branch-width for hypergraphs in [28]. Roughly speaking, a hypergraph has small branch-width if it can be decomposed into small pieces along low-order separations in a tree-like way. One of the attractive properties of branch-width is that it easily extends to connectivity functions as follows.

Let $\lambda$ be a connectivity function on a finite set $E$. A tree is cubic if all its internal vertices have degree 3 . A branch-decomposition $(T, \tau)$ of $\lambda$ consists of a cubic tree $T$ and a bijection $\tau$ from the set of leaves of $T$ to the set $E$. If $e$ is an edge of $T$, then $T \backslash e$ induces a partition $(X, Y)$ of the set of leaves of $T$. The width of $e$ is defined to be $\lambda(\tau(X))$. The width of the branchdecomposition $(T, \tau)$ is the maximum among the widths of the edges of $T$. 
The branch-width of $\lambda$, denoted branch-width $(\lambda)$, is the minimum among the widths of the branch-decompositions of $\lambda$. If $\lambda$ has no branch-decompositions, then we define the branch-width of $\lambda$ to be $\lambda(\emptyset)$.

If $G$ is a hypergraph, then the branch-width of $G$, denoted branch-width $(G)$, is defined to be the branch-width of its connectivity function $\lambda_{G}$. Similarly, if $M$ is a matroid, then the branch-width of $M$, denoted branch-width $(M)$, is defined to be the branch-width of its connectivity function $\lambda_{M}$. Since $\lambda_{M}=\lambda_{M^{*}}$ for any matroid $M$, we see that branch-width for matroids is invariant under duality.

Robertson and Seymour proved that a hypergraph has bounded treewidth if and only if it has bounded branch-width. More precisely, they proved the following theorem.

Theorem 2.2.1. [28, 5.2] If $G$ is a hypergraph, then

$$
\operatorname{branch-width}(G) \leq \operatorname{tree-width}(G)+1 \leq \frac{3}{2} \operatorname{branch-width}(G) .
$$

Similarly, it was proved by Hliněný and Whittle in [19] that a matroid has bounded tree-width if and only if it has bounded branch-width, though the precise bounds for matroids differ from those given in Theorem 2.2.1.

Given that branch-width can be defined for both graphs and matroids, it is natural to ask if the branch-width of a graph is equal to the branch-width of its cycle matroid. In general, the branch-width of a graph may be larger than the branch-width of its cycle matroid. For example, consider a path of length 3. However, if a graph $G$ has a cycle of length at least 2, then $G$ and its cycle matroid $M(G)$ have the same branch-width [17]. Combining this result with the fact that branch-width for matroids is invariant under duality, one easily sees that the branch-width of a planar graph containing a cycle is equal to the branch-width of its geometric dual. Note that this seemingly natural result does not hold for tree-width. For example, the cube and the octahedron have different tree-width.

We now conclude this section with a discussion of some of the interesting properties of branch-width for matroids. 
Perhaps the most significant use of branch-width for matroids is in the work of Geelen, Gerards, and Whittle which aims to extend the results and techniques of Robertson and Seymour's Graph-Minors Project to matroids. In [8], Geelen, Gerards, and Whittle prove that, for any prime power $q$ and any positive integer $n$, the class of $\mathrm{GF}(q)$-representable matroids with branchwidth at most $n$ is well-quasi-ordered under the minor relation. The theorem also has consequences for the excluded minors of $\mathrm{GF}(q)$-representable matroids. Indeed, Geelen and Whittle [15] have proved that, for each positive integer $k$ and each prime power $q$, the class of $\mathrm{GF}(q)$-representable matroids has only finitely many excluded minors of branch-width at most $k$.

The class $\mathcal{B}_{k}$ of matroids of branch-width at most $k$ has many attractive properties. Indeed, $\mathcal{B}_{k}$ is closed under taking duals, minors, direct sums, and 2 -sums. Therefore it is natural to seek excluded-minor characterisations for $\mathcal{B}_{k}$. The matroids of branch-width one are precisely the direct sums of loops and coloops, so $U_{1,2}$ is the unique excluded minor for $\mathcal{B}_{1}$. The matroids of branch-width at most 2 are the series-parallel matroids [28]; and the excluded minors are known to be $U_{2,4}$ and $M\left(K_{4}\right)$ [22]. For all $k \geq 3$, however, no complete list of excluded minors for $\mathcal{B}_{k}$ is known. For the binary matroids of branch-width 3, Dharmatilake [5] gave a list of 10 excluded minors, and conjectured that his list was complete. Dharmatilake's conjecture was later proved by Hliněný [18]. Hall, Oxley, Semple, and Whittle [16] proved that there are finitely many excluded minors for $\mathcal{B}_{3}$. For each positive integer $k \geq 2$, Geelen, Gerards, Robertson, and Whittle [7] proved that an excluded minor for $\mathcal{B}_{k}$ has size at most $\left(6^{k}-1\right) / 5$, thus proving that $\mathcal{B}_{k}$ has a finite number of excluded minors.

\subsubsection{Tangles}

Finite graphs and $\operatorname{GF}(q)$-representable matroids of bounded branch-width are well-quasi-ordered under the minor relation [27, 8]. It remains, then, to consider those graphs and $\mathrm{GF}(q)$-representable matroids of large branchwidth. Robertson and Seymour [26] proved that such graphs contain a large 
grid minor. Similarly, Geelen, Gerards, and Whittle [1] proved that GF $(q)$ representable matroids with large branch-width contain the cycle matroid of a large grid as a minor. The existence of a large grid-minor is useful for investigating the structure of the graphs and $\operatorname{GF}(q)$-representable matroids of large branch-width. However, a graph or $\operatorname{GF}(q)$-representable matroid may have several regions of large branch-width that are separated by low-order separations. To gain a handle on these parts, Robertson and Seymour [28] introduced tangles.

Tangles are the canonical obstruction to small branch-width, that is, a structure that appears in a graph or matroid if and only if the branch-width is large. Loosely speaking, a tangle indicates for each low-order separation on which side a particular high branch-width part lies. Although Robertson and Seymour introduced tangles for hypergraphs, the definition of a tangle extends naturally to arbitrary connectivity systems as follows.

Let $\lambda$ be a connectivity function on $E$, and let $k$ be a positive integer. A tangle of order $k$ in $(E, \lambda)$ is a collection $\mathcal{T}$ of subsets of $E$ such that the following properties hold:

(T1) $\lambda(A)<k$ for all $A \in \mathcal{T}$.

(T2) If $(A, E-A)$ is a $(k-1)$-separation, then $\mathcal{T}$ contains $A$ or $E-A$.

(T3) If $A, B, C \in \mathcal{T}$, then $A \cup B \cup C \neq E$.

(T4) $E-\{e\} \notin \mathcal{T}$ for each $e \in E$.

We note that (T3) can be used to sharpen (T2) to say that $\mathcal{T}$ contains exactly one of $A$ or $E-A$.

If $G$ is a hypergraph, then tangle of order $k$ in $G$ is a tangle of order $k$ in its connectivity system $\left(E(G), \lambda_{G}\right)$. Similarly, if $M$ is a matroid, then tangle of order $k$ in $M$ is a tangle of order $k$ in its connectivity system $\left(E(M), \lambda_{M}\right)$.

Robertson and Seymour [28] proved that the branch-width of a hypergraph $G$ is equal to the maximum order of a tangle in $G$. Dharmatilake [6] defined tangles for matroids, and proved that the branch-width of a matroid 
$M$ is equal to the maximum order of a tangle in $M$. Geelen, Gerards, Robertson, and Whittle [9] defined tangles for arbitrary connectivity functions, and proved the following result.

Theorem 2.2.2. [9, Theorem 3.2] Let $\lambda$ be a connectivity function on E. Then the maximum order of a tangle in $(E, \lambda)$ is equal to the branch-width of $\lambda$.

We note that, although they did not explicitly define tangles for connectivity functions, Theorem 2.2.2 is cryptomorphic to a result proved by Robertson and Seymour [28, (3.5)].

Tangles are a central component to Robertson and Seymour's GraphMinor Structure Theorem [29]. Loosely speaking, the Graph-Minor Structure Theorem states that any graph $G$ without a fixed graph $H$ as a minor admits a tree-decomposition into pieces that almost embed into some surface that $H$ does not. The pieces of the tree-decomposition of a graph correspond to its maximal tangles, that is, the tangles that are inclusion-wise maximal. Roughly, the tree-decomposition enables one to convert information about the 'local structure' of a graph relative to each maximal tangle into knowledge of the 'global structure' of a graph.

Geelen, Gerards, and Whittle [13] proved that any connectivity function can be put together from its maximal tangles in a tree-like way. We now describe this decomposition in more detail. Let $\lambda$ be a connectivity function on a set $E$. A tree-decomposition of $\lambda$ consists of a tree $T$ and a partition $\pi$ of $E$ whose members, called bags, label the vertices of $T$. A $k$-separation $(X, Y)$ of $\lambda$ is displayed by a tree-decomposition $T$ of $\lambda$ if, for some edge $e$ of $T$, the set $X$ is the union of the bags labelling the vertices of a component of $T \backslash e$. Let $\mathcal{T}_{1}$ and $\mathcal{T}_{2}$ be distinct tangles in a connectivity function $\lambda$. Then $\left(X_{1}, X_{2}\right)$ is a distinguishing separation if $X_{1} \in \mathcal{T}_{1}$ and $X_{2} \in \mathcal{T}_{2}$. We now state the main result.

Theorem 2.2.3. [13, Theorem 9.1] Let $\lambda$ be a connectivity function on E, and let $\mathcal{T}_{1}, \ldots \mathcal{T}_{n}$ be maximal tangles in $\lambda$. Then there exists a tree decompo- 
sition $T$ of $\lambda$ such that $V(T)=[n]$ and such that the following hold:

(i) For each $i \in V(T)$ and $e \in E(T)$ if $T^{\prime}$ is the component of $T \backslash e$ containing $i$, then the union of those bags that label vertices of $T^{\prime}$ is not a member of $\mathcal{T}_{i}$.

(ii) For each pair of distinct vertices $i$ and $j$ of $T$, there exists a minimumorder distinguishing separation for $\mathcal{T}_{i}$ and $\mathcal{T}_{j}$ that is displayed by $T$.

Theorem 2.2.3 extends a key result [28, 10.3] of Robertson and Seymour for graphs. Geelen, Gerards, and Whittle also proved, as a corollary of Theorem 2.2.3, that the number of maximal tangles in an $m$-element connectivity system is at most $(m-2) / 2$.

We conclude this section with a basic result on tangles in $k$-connected matroids.

Lemma 2.2.4. [6, Lemma 2.4] Let $M$ be a matroid, and let $k \geq 2$. If $M$ is $k$-connected and $|E(M)| \geq 3(k-2)+1$, then $\mathcal{T}=\{X \subseteq E(M)|| X \mid \leq k-2\}$ is the unique tangle of order $k$ in $M$.

We will use special cases of Lemma 2.2.4 later for illustration. We defer a proof to Section 5.1 .

\subsubsection{Tangles of order three in connected matroids}

We now sketch a tangle-theoretic approach to the well-known 2-sum decomposition for connected matroids that may help the reader's intuition for tangles. We work with a connected matroid $M$ and a fixed tangle $\mathcal{T}$ of order three in $M$. A set $X \in \mathcal{T}$ is a maximal member of $\mathcal{T}$ if no set that properly contains $X$ is in $\mathcal{T}$.

Recall that Cunningham and Edmonds [4] described the following treelike decompostion for the connected matroids that are not 3-connected which gives an explicit description of their structure. 
Theorem 2.2.5. [22, Proposition 8.3.5] Let $M$ be a connected matroid. Then, for some integer $k$, there are 3-connected matroids $M_{1}, \ldots, M_{k}$ and a $k$-vertex tree $T$ with edges $e_{1}, \ldots, e_{k-1}$ and vertices labelled by $M_{1}, \ldots, M_{k}$ such that

(i) $E\left(M_{1}\right) \cup \cdots \cup E\left(M_{k}\right)=E(M) \cup\left\{e_{1}, \ldots, e_{k-1}\right\}$;

(ii) if $e_{i}$ joins $M_{a}$ and $M_{b}$, then $M_{a} \cap M_{b}=\left\{e_{i}\right\}$; and

(iii) if no edge joins $M_{a}$ and $M_{b}$, then $M_{a} \cap M_{b}=\emptyset$.

Moreover, $M$ is the matroid that labels the single vertex of $T /\left\{e_{1}, \ldots, e_{k-1}\right\}$ at the conclusion of the following process: contract the edges $e_{1}, \ldots, e_{k-1}$ one by one in order; when $e_{i}$ is contracted, its ends are identified and the vertex formed by this identification is labelled by the 2-sum of the matroids that previously labelled the ends of $e_{i}$.

We show that each tangle $\mathcal{T}$ of order three in a connected matroid $M$ corresponds to a component in the 2-sum decomposition of $M$. We begin by showing that the maximal members of a tangle $\mathcal{T}$ of order three in $M$ partition $E(M)$.

Lemma 2.2.6. Let $\mathcal{T}$ be a tangle of order 3 in a connected matroid $M$. Then the maximal members of $\mathcal{T}$ partition $E(M)$.

Proof. We first show that the maximal members of $\mathcal{T}$ cover $E(M)$. Let $e \in E(M)$. Then $\lambda(\{e\})<3$, so $\{e\} \in \mathcal{T}$ by (T4). Thus, $e$ is contained in some maximal member of $\mathcal{T}$.

We now show that the maximal members of $\mathcal{T}$ are pairwise disjoint. Assume that $X$ and $Y$ are distinct maximal members of $\mathcal{T}$. If $X \cap Y$ is nonempty, then $\lambda(X \cap Y) \geq 2$ because $M$ is connected. Hence $\lambda(X \cup Y) \leq 2$ by the submodularity of $\lambda$. By (T2), either $X \cup Y$ or $E-(X \cup Y)$ is a member of $\mathcal{T}$. But $X \cup Y$ is not a member of $\mathcal{T}$ because $X$ are $Y$ are maximal members of $\mathcal{T}$. Thus $E-(X \cup Y)$ is a member of $\mathcal{T}$. Then $X, Y$, and $E-(X \cup Y)$ cover $E(M)$; a contradiction of (T3). Thus $X \cap Y$ is empty and the lemma is proved. 
We note that Lemma 2.2.6 does not hold for tangles of order $k>3$.

Let $M$ be a matroid. For a set $X=\left\{X_{1}, X_{2}, \ldots, X_{n}\right\}$ of subsets of $E(M)$ we define a function $\rho_{M}$ as follows

$$
\rho_{M}(X)=r_{M}\left(X_{1} \cup X_{2} \cup \cdots \cup X_{n}\right)-\sum_{i=1}^{n} r_{M}\left(X_{i}\right)+n .
$$

Let $T$ be the set of maximal members of a tangle $\mathcal{T}$ of order 3 in a connected matroid $M$. We define a set function $\rho_{\mathcal{T}}$ on $T$ by letting $\rho_{\mathcal{T}}(X)=$ $\rho_{M}(X)$ for all $X \subseteq T$.

Let $X$ and $Y$ be disjoint subsets of $E(M)$. We define

$$
\kappa_{M}(X, Y)=\min \left\{\lambda_{M}(Z) \mid X \subseteq Z \subseteq E(M)-Y\right\}
$$

The following theorem was proved by Tutte [31].

Theorem 2.2.7. (Tutte's Linking Theorem.) If $X$ and $Y$ are disjoint sets in a matroid $M$, then there exists a minor $N$ of $M$ such that $E(N)=X \cup Y$ and $\lambda_{N}(X)=\kappa_{M}(X, Y)$.

We sketch the proof of the next result.

Lemma 2.2.8. Let $T=\left(T_{1}, T_{2}, \ldots, T_{n}\right)$ be the maximal members of a tangle $\mathcal{T}$ of order 3 in a connected matroid $M$, and let $\left(t_{1}, t_{2}, \ldots, t_{n}\right)$ be a transversal of $T$.

(i) $\rho_{\mathcal{T}}$ is the rank function of a 3-connected matroid $N$ on $T$.

(ii) $M$ has a minor on $\left\{t_{1}, t_{2}, \ldots, t_{n}\right\}$ that is isomorphic to $N$.

(iii) If $\left\{X_{1}, \ldots, X_{m}\right\}$ and $\left\{Y_{1}, \ldots, Y_{p}\right\}$ are disjoint subsets of $T$, then $\kappa_{N}\left(\left\{X_{1}, \ldots, X_{m}\right\},\left\{Y_{1}, \ldots, Y_{p}\right\}\right)=\kappa_{M}\left(X_{1} \cup \cdots \cup X_{m}, Y_{1} \cup \cdots \cup Y_{p}\right)$.

Proof. If each member of $T$ has one element, then the result is immediate. Assume that $\left|T_{1}\right| \geq 2$. Applying Tutte's Linking Theorem to the disjoint subsets $E(M)-T_{1}$ and $\left\{t_{1}\right\}$, there is a minor $N$ of $M$ such that $E(N)=\left(E(M)-T_{1}\right) \cup\left\{t_{1}\right\}$ and $\lambda_{N}\left(\left\{t_{1}\right\}\right)=2$. It is easily checked that 
$T^{\prime}=\left\{\left\{t_{1}\right\}, T_{2}, \ldots, T_{n}\right\}$ is the set of maximal members of a tangle $\mathcal{T}^{\prime}$ of order three in $N$. By induction we may assume that the lemma holds for $T^{\prime}$ in $N$, and one routinely checks that the lemma also holds for $T$ in $M$.

The matroid $N$ in Lemma 2.2 .8 is called the matroid induced by $\mathcal{T}$. We may regard its ground set as being the maximal members of $\mathcal{T}$ or some transversal of these sets according to convenience. It is isomorphic to one of the components in the 2-sum decomposition of $M$. Indeed, the tangles of order three correspond to the different components of the 2-sum decomposition described in Theorem 2.2.5.

Suppose that $M$ is 3 -connected up to series or parallel classes. Then $M$ has a unique tangle of order 3 , and the matroid induced by this tangle is the simplification or cosimplification of $M$ respectively. In general, a connected matroid has a unique tangle of order three if and only if it has the property that whenever $(A, B)$ is a 2-separation of $M$ with corresponding 2-sum decomposition $M_{A} \oplus_{2} M_{B}$, then either $M_{A}$ or $M_{B}$ is a series-parallel matroid.

\subsection{The structure of the 3-separations of 3 - connected matroids}

The tree decomposition for 3-connected matroids described by Oxley, Semple, and Whittle [23, 24] has become an important tool in matroid structure theory. Their work was particularly influential in the development of this thesis, so we provide a summary of their work here as background. Loosely speaking, the main theorem of [23] states that, for a 3 connected matroid $M$ having at least nine elements, there is a labelled tree with the property that, up to equivalence, all "non-sequential" 3-separations of $M$ are displayed by $T$. The main theorem of [24] shows that, if certain conditions are imposed on the tree in [23], then it has a uniqueness property. 


\subsubsection{Sequential and equivalent 3-separations}

An important feature of the tree decompostion described by Oxley, Semple, and Whittle is that it guarantees to display only the "non-sequential" 3separations, and of these, only a member from each equivalence class. We first make these notions precise, and then we illustrate, by way of example, why the feature is necessary.

Let $M$ be a 3-connected matroid. A set $X$ in $M$ is fully closed if it is closed in both $M$ and $M^{*}$, that is, $X=\operatorname{cl}(X)$ and $X=\mathrm{cl}^{*}(X)$. The full closure of a subset $X$ of a matroid $M$, denoted $\mathrm{fcl}(X)$, is the intersection of all fully closed sets containing $X$. We can use the full closure operator to define an equivalence relation on the set of 3-separations of $M$ as follows. Let $(A, B)$ and $(C, D)$ be exact 3 -separations of $M$. Then $(A, B)$ and $(C, D)$ are equivalent if $\{\mathrm{fcl}(A), \mathrm{fcl}(B)\}=\{\mathrm{fcl}(C), \mathrm{fcl}(D)\}$. An exact 3-separation $(A, B)$ is sequential if $\mathrm{fcl}(A)=E(M)$ or $\mathrm{fcl}(B)=E(M)$.

Consider the following examples from [23]. Let $P$ be the matroid $P G(3, q)$ for some prime power $q$. If $L$ is a line of $P$, then it is easily seen that $(L, E(P)-L)$ is a sequential 3-separation of $P$. Clearly there is no way to display all of the lines of $P$ in a tree-like way.

Now suppose that $P_{1}$ and $P_{2}$ are distinct planes of $P$, and consider the matroid $P^{\prime}=P \mid\left(P_{1} \cup P_{2}\right)$. Let $A=P_{1}-P_{2}, B=P_{1} \cap P_{2}$, and $C=P_{2}-P_{1}$. It is easily seen that, for all subsets $B^{\prime}$ of $B$, the partition $\left(A \cup B^{\prime}, C \cup\left(B-B^{\prime}\right)\right)$ is a 3-separation of $P^{\prime}$. Moreover, it is easily seen that all of these 3 -separations are equivalent. Clearly there is no reasonable way to display all of them in a tree-like way.

We have seen that from a structural point of view, the existence of equivalent and sequential 3-separations is not problematic; they can be classified by the full closure operator. The existence of equivalent and sequential 3separations is also not problematic from an algorithmic point of view; if one had a rank oracle, then listing all sequential 3-separations or all of the 3separations equivalent to a given 3 -separation can done so that each item on the list is added in polynomial time (see [24]). 


\subsubsection{Flowers}

One of the difficulties of characterising the 3-separations of 3-connected matroids is that they can "cross". Two 3 -separations $(A, B)$ and $(C, D)$ cross if all the intersections $A \cap C, A \cap D, B \cap C$, and $B \cap D$ are non-empty. Flowers are the fundamental structures that were introduced in [23] to study collections of mutually crossing 3 -separations.

Let $n$ is a positive integer, and let $M$ be a 3 connected matroid. A partition $\Phi=\left(P_{1}, \ldots, P_{n}\right)$ of $E(M)$ is a flower with petals $P_{1}, \ldots, P_{n}$ if, for all $i \in[n],\left|P_{i}\right| \geq 2$, and both $P_{i}$ and $P_{i} \cup P_{i+1}$ are 3 -separating, where subscripts are interpreted modulo $n$.

Understanding the structure of flowers was crucial to obtaining a tree that displays all of the non-sequential 3-separations of a 3-connected matroid, so we now describe the classes of flowers. A flower $\Phi$ is an anemone if any union of petals of $\Phi$ is 3-separating, and a daisy if only consecutive unions of petals of $\Phi$ in the cyclic order are 3-separating. The classes of daisies and anemones can be further refined according to the local connectivity between pairs of petals. For sets $X, Y \subseteq E(M)$, the local connectivity between $X$ and $Y$, denoted $\sqcap(X, Y)$, is defined by $\sqcap(X, Y)=r(X)+r(Y)-r(X \cup Y)$.

For $n \geq 3$, an anemone $\left(P_{1}, \ldots, P_{n}\right)$ is called

(i) a paddle if $\sqcap\left(P_{i}, P_{j}\right)=2$ for all $i \neq j$;

(ii) a copaddle if $\sqcap\left(P_{i}, P_{j}\right)=0$ for all $i \neq j$; and

(iii) spike-like if $n \geq 4$, and $\sqcap\left(P_{i}, P_{j}\right)=1$ for all $i \neq j$.

Similarly, a daisy $\left(P_{1}, \ldots, P_{n}\right)$ is called

(i) swirl-like if $n \geq 4$ and $\sqcap\left(P_{i}, P_{j}\right)=1$ for all consecutive $i, j$, while $\sqcap\left(P_{i}, P_{j}\right)=0$ for all non-consecutive $i, j$; and

(ii) Vámos-like if $n=4$ and $\sqcap\left(P_{i}, P_{j}\right)=1$ for all consecutive $i, j$, while $\left\{\sqcap\left(P_{1}, P_{3}\right), \sqcap\left(P_{2}, P_{4}\right)\right\}=\{0,1\}$. 
Finally, a flower $\left(P_{1}, \ldots, P_{n}\right)$ is unresolved if $n=3$, and $\sqcap\left(P_{i}, P_{j}\right)=1$ for all $i \neq j$.

The next theorem shows that every flower with at least three petals is one of these types.

Theorem 2.3.1. [23, Theorem 4.1] If $\Phi=\left(P_{1}, \ldots, P_{n}\right)$ is a flower, then $\Phi$ is either a daisy or an anemone. Moreover, if $n \geq 3$, then $\Phi$ is either a paddle, a copaddle, spike-like, swirl-like, Vámos-like, or is unresolved.

Following [23], we now give an informal description of some flowers. We visualise a flower by thinking of a collection of lines in projective space. These lines can be thought of as lines of attachment of 3-separating sets that form the petals of the flower.

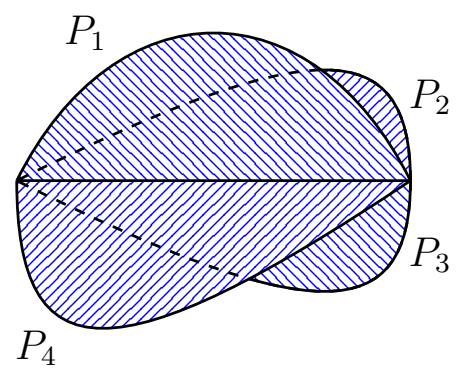

Figure 2.1: A representation of a rank-6 paddle.

Example 7. Consider a collection of rank-3 planes $P_{1}, \ldots, P_{n}$ glued together along a single common line, and assume that each plane has enough structure to make the resulting matroid 3-connected. The resulting partition $\left(P_{1}, \ldots, P_{n}\right)$ is an paddle of $\operatorname{rank} \sum_{i=1}^{n} r\left(P_{i}\right)-2(n-1)$. We illustrate a 4-petal rank-6 paddle in figure 2.1 .

Example 8. Choose an independent set $\left\{p_{1}, \ldots, p_{n}\right\}$ in projective space, and let $L_{i}$ be the line spanned by $\left\{p_{i}, p_{i+1}\right\}$ for all $i$, modulo $n$. Attach rank-3 planes $P_{i}$ to $L_{i}$ for all $i$, again assuming each plane has enough structure 


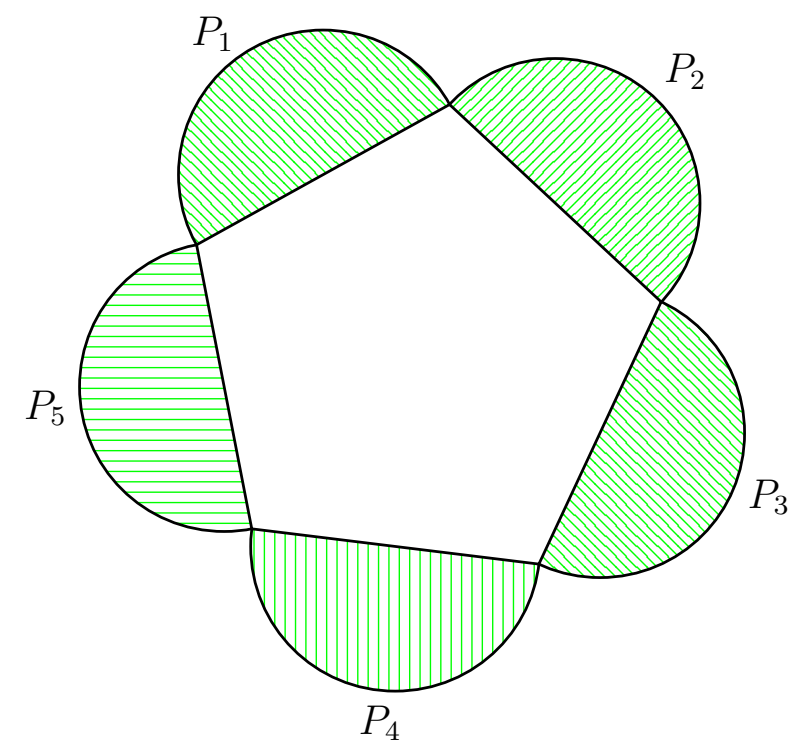

Figure 2.2: A representation of a rank-10 swirl-like flower.

to make the resulting matroid 3 -connected. The partition $\left(P_{1}, \ldots, P_{n}\right)$ is a swirl-like flower of rank $\sum_{i=1}^{n} r\left(P_{i}\right)-n$. We illustrate a 5 -petal rank-10 swirl-like flower in figure 2.2 .

A flower $\Phi$ displays a 3 -separating set $X$ or a 3 -separation $(X, Y)$ if $X$ is a union of petals of $\Phi$. Recall that a quasi-order is a reflexive and transitive relation. There is a natural quasi-order $\preccurlyeq$ on the set of flowers in $M$ induced by the non-sequential 3-separations that they display, and this order gives rise to a natural notion of equivalence of flowers. Let $\Phi_{1}$ and $\Phi_{2}$ be flowers in $M$. Then $\Phi_{1} \preccurlyeq \Phi_{2}$ if, for every non-sequential 3-separation displayed by $\Phi_{1}$, there is an equivalent one displayed by $\Phi_{2}$. Two flowers $\Phi_{1}$ and $\Phi_{2}$ are equivalent if both $\Phi_{1} \preccurlyeq \Phi_{2}$ and $\Phi_{2} \preccurlyeq \Phi_{1}$. Thus equivalent flowers display, up to equivalence of 3 -separations of $M$, the same non-sequential 3 -separations. The order of a flower $\Phi$ is the minimum number of petals in a flower equivalent to $\Phi$.

We have seen that equivalent flowers display, up to equivalence of 3 - 
separations, the same non-sequential 3-separations. The flowers that contain the minimum number of petals among equivalent flowers are "tight", and understanding their structure turns out to be important. An element $e$ of $M$ is loose in the flower $\Phi$ if $e \in \operatorname{fcl}\left(P_{i}\right)-P_{i}$ for some petal $P_{i}$ of $\Phi$. An element $e$ of $M$ that is not loose in $\Phi$ is called tight in $\Phi$. The petal $P_{i}$ of $\Phi$ is loose if every element in $P_{i}$ is loose. A petal of $\Phi$ is tight if it is not loose. Thus a tight petal contains at least one element that is tight in $\Phi$. A flower of order at least 3 is tight if all of its petals are tight. A flower of order 1 or 2 is tight if it has one petal or two petals respectively. Oxley, Semple, and Whittle prove that the order of a flower $\Phi$ is equal to the number of petals in any tight flower equivalent to $\Phi$ [23, Theorem 5.1].

A flower is maximal if it is maximal in the quasi-order $\preccurlyeq$. Proving that the non-sequential 3-separations of a matroid "conform" with tight maximal flowers was crucial in the proof of the main theorem. We state the theorem after the next definition. Let $(X, Y)$ be a 3 -separation of $M$. We say that $(X, Y)$ conforms with $\Phi$ if either $(X, Y)$ is equivalent to a 3-separation displayed by $\Phi$, or $(X, Y)$ is equivalent to a 3 -separation $\left(X^{\prime}, Y^{\prime}\right)$ such that $X^{\prime}$ or $Y^{\prime}$ is contained in a petal of $\Phi$.

Theorem 2.3.2. [23, Theorem 8.1] Let $M$ be a 3-connected matroid with at least 9 elements, and let $\Phi$ be a tight maximal flower in $M$. Then every non-sequential 3-separation of $M$ conforms with $\Phi$.

The condition that the matroid has at least 9 elements in Theorem 2.3.2 is essential, as the following example from [23] shows. Consider the 8-element rank-4 matroid $R_{8}$ that is represented geometrically by a cube (see [22, pp. 508]). The 4-point planes of $R_{8}$ are the six faces of the cube and the six diagonal planes. With notation as in figure 2.3, the partition $\Phi=(\{1,2\},\{3,4\},\{5,6\},\{7,8\})$ is a tight maximal flower. However, the non-sequential 3 -separation $(\{1,3,5,7\},\{2,4,6,8\})$ does not conform with $\Phi$. 


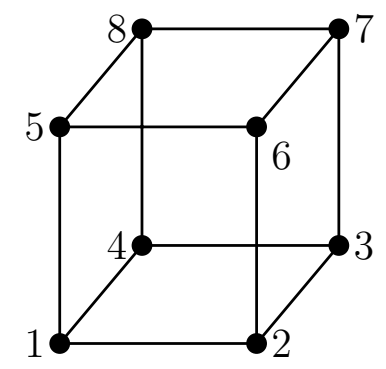

Figure 2.3: The matroid, $R_{8}$.

\subsubsection{Partial 3-trees}

We now define the tree structure described in [23]. Let $\pi$ be a partition of a finite set $E$. Let $T$ be a tree such that every member of $\pi$ labels some vertex of $T$. Note that some vertices may be unlabelled, and that no vertex is multiply labelled. Then $T$ is called a $\pi$-labelled tree. Labelled vertices are called bag vertices and the members of $\pi$ are called bags.

Let $T^{\prime}$ be a subtree of $T$. The union of bags that label vertices of $T^{\prime}$ is called the subset of $E$ displayed by $T^{\prime}$. The partition of $E$ displayed by $e$ is the partition displayed by the connected components of $T \backslash e$. Let $v$ be a vertex of $T$ that is not a bag vertex. Then the partition of $E$ displayed by $v$ is the partition displayed by the connected components of $T-v$. The edges incident with $v$ are in natural one-to-one correspondence with the connected components of $T-v$, and hence with the members of the partition of $E$ displayed by $v$. In what follows, if a cyclic ordering is imposed on the edges incident with $v$, then we cyclically order the members of the partition of $E$ displayed by $v$ in the corresponding order.

Let $M$ be a 3-connected matroid on ground set $E$. An almost partial 3 -tree $T$ for $M$ is a $\pi$-labelled tree, where $\pi$ is a partition of $E$ such that the following conditions hold:

(i) For each edge $e$ of $T$, the partition $(X, Y)$ of $E$ displayed by $e$ is 3separating, and, if $e$ is incident with two bag vertices, then $(X, Y)$ is a 
non-sequential 3-separation.

(ii) Every non-bag vertex $v$ is labelled either $D$ or $A$. If $v$ is labelled $D$, then there is a cyclic ordering on the edges incident with $v$.

(iii) If a vertex $v$ is labelled $A$, then the partition of $E$ displayed by $v$ is a tight maximal anemone of order at least 3 .

(iv) If a vertex $v$ is labelled $D$, then the partition of $E$ displayed by $v$, with the cyclic order induced by the cyclic ordering on the edges incident with $v$, is a tight maximal daisy of order at least 3 .

By conditions (iii) and (iv), a vertex $v$ labelled $D$ or $A$ corresponds to a flower of $M$. The 3-separations displayed by this flower are the 3-separations displayed by $v$. A vertex of a partial 3-tree is referred to as a daisy vertex or an anemone vertex if it is labelled $D$ or $A$, respectively. A vertex labelled either $D$ or $A$ is a flower vertex. A 3-separation is displayed by an almost partial 3-tree $T$ if it is displayed by some edge or some flower vertex of $T$. A 3-separation $(R, G)$ of $M$ conforms with an almost partial 3-tree $T$ if either $(R, G)$ is equivalent to a 3 -separation that is displayed by a flower vertex or an edge of $T$, or $(R, G)$ is equivalent to a 3-separation $\left(R^{\prime}, G^{\prime}\right)$ such that $R^{\prime}$ or $G^{\prime}$ is contained in a bag of $T$.

An almost partial 3-tree for $M$ is a partial 3-tree if every non-sequential 3 -separation of $M$ conforms with $T$. The set of partial 3-trees for $M$ is quasi-ordered as follows. Let $T_{1}$ and $T_{2}$ be two partial 3 -trees for $M$. Then $T_{1} \preccurlyeq T_{2}$ if, for every non-sequential 3 -separations displayed by $T_{1}$, there is some equivalent 3 -separation displayed by $T_{2}$. If $T_{1} \preccurlyeq T_{2}$ and $T_{2} \preccurlyeq T_{1}$, then $T_{1}$ is an equivalent partial 3 -tree to $T_{2}$. A partial 3 -tree is maximal if it is maximal in the quasi-order $\preccurlyeq$.

We now state the main result of [23].

Theorem 2.3.3. [23, 9.1] Let $M$ be a 3-connected matroid with $|E(M)| \geq 9$, and let $T$ be a maximal partial 3-tree for $M$. Then every non-sequential 3separation of $M$ is equivalent to a 3-separation displayed by $T$. 
Maximal partial 3-trees are by no means unique. Consider the following example from [24]. Let $M$ be the 3-connected matroid obtained by taking three distinct triangles in $M\left(K_{4}\right)$ and, along each, attaching a copy of $F_{7}$ by generalised parallel connection. One maximal partial 3-tree for $M$ consists of a bag vertex that is labelled by the elements of $M\left(K_{4}\right)$ and is adjacent to exactly three other bag vertices, each labelled by the elements of one of the copies of $F_{7}$ that are not in $M\left(K_{4}\right)$. Another maximal partial 3-tree for $M$ consists of a flower vertex that is adjacent to exactly three bag vertices, each labelled by the elements of one of the copies of $F_{7}$.

To get a more canonical structure, Oxley, Semple, and Whittle [24] introduced the notion of a 3 -tree. A maximal partial 3-tree for $M$ is a 3-tree if

(i) for every tight maximal flower of $M$ of order three, there is an equivalent flower that is displayed by a vertex of $T$; and

(ii) if a vertex is incident with two edges, $e$ and $f$, that display equivalent 3separating partitions, then the other ends of $e$ and $f$ are flower vertices, $v$ has degree two, and $v$ labels a non-empty bag.

Two edges of a 3-tree are twins if they are incident with a common vertex and display equivalent 3 -separations. The reduction of a 3 -tree $T$, denoted $R(T)$, is the unlabelled tree obtained from $T$ by contracting one edge from each pair of twins of $T$.

We now summarise the main results from [24].

Theorem 2.3.4. Let $M$ be a 3-connected matroid with $|E(M)| \geq 9$. Then $M$ has a 3-tree $T$. Moreover, if $T_{1}$ and $T_{2}$ are 3 -trees for $M$, then $T_{1}$ and $T_{2}$ have isomorphic reductions.

\section{$2.4 \quad$ Higher-order separations}

In this section, we review the work of Aiken and Oxley [2] in describing the structure of crossing $k$-separations of arbitrary matroids. We then ex- 
amine the notion of 2-equivalence of 4 -separations of 4-connected matroids defined by Aikin in [1]. Though we have not seen their work, Aikin and Oxley (private communication) have described a tree decomposition of a 4connected matroid $M$ that displays, up to 2-equivalence, the "non-sequential" 4-separations of $M$.

\subsection{1 $k$-flowers}

Given the success of flowers in describing the interactions of crossing 3separations in 3-connected matroids, it is natural to ask if flowers can be extended to describe the interactions of higher-order crossing separations in more general structures. Indeed, this was done by Aiken and Oxley [2], who defined a generalised flower structure called a $k$-flower to study the behaviour of crossing $k$-separations in arbitrary matroids.

Let $\lambda$ be a connectivity function on a finite set $E$, and let $k$ be a positive integer. A $k$-flower in $\lambda$ with petals $P_{1} \ldots, P_{n}$ is a partition $\left(P_{1}, \ldots, P_{n}\right)$ of $E$ such that, for all $i$, both $P_{i}$ and $P_{i} \cup P_{i+1}$ are exact $k$-separating sets, where subscripts are interpreted modulo $n$.

Let $\Phi=\left(P_{1}, \ldots, P_{n}\right)$ be a $k$-flower in $\lambda$. Then $\Phi$ is a $k$-anemone if any union of petals of $\Phi$ is $k$-separating, and $\Phi$ is a $k$-daisy if only consecutive unions of petals of $\Phi$ in the cyclic order are $k$-separating.

Most of [2] is concerned with extending the complete flower classification described in Theorem 2.3.1 to polymatroids. Recall that a polymatroid on a set $E$ is an integer-valued, increasing, submodular function on the subsets of $E$ whose value on $\emptyset$ is 0 . The connectivity function $\lambda_{f}$ of a polymatroid $f$ on $E$ is defined for all $X \subseteq E$ by $\lambda_{f}(X)=f(X)+f(E-X)-f(E)+1$. The local connectivity in $f$ between subsets $X$ and $Y$ of $E$, denoted $\sqcap_{f}(X, Y)$, is defined by $\sqcap_{f}(X, Y)=f(X)+f(Y)-f(X \cup Y)$. We can now summarise the main results of [2].

Theorem 2.4.1. Let $\lambda$ be a connectivity function on $E$, and let $\Phi=$ $\left(P_{1}, \ldots, P_{n}\right)$ be a $k$-flower in $\lambda$ for some $k \geq 1$. Then $\Phi$ is either a $k$ - 
anemone or a $k$-daisy. Moreover, if $n \geq 4$ and $\lambda$ is the connectivity function of a polymatroid, then $\Phi$ can be completely classified in terms of $n, k$, the local connectivity between consecutive petals, and the local connectivity between non-consecutive petals.

Aikin and Oxley [2] also construct examples of $k$-flowers in matroids to satisfy each of the possibilities given in their classification theorem.

\subsubsection{The structure of the 4-separations of 4-connected matroids}

Let $M$ be a 4-connected matroid. Aikin [1] observed that, to have any hope of displaying the 4-separations of $M$ in a tree-like way, one could only guarantee to display the "non-sequential" 4-separations up to some notion of equivalence. Furthermore, he observed that, unlike the situation for 3separations, any natural equivalence of the 4-separations must incorporate 2-element "equivalence moves". To characterise these notions, Aikin defined the following extension of the full closure operator.

The full 2-span of a subset $X$ of $M$, denoted $\mathrm{fs}_{2}(X)$, is the set $X \cup X_{1} \cup$ $\cdots \cup X_{m}$, where $X_{1}, \ldots, X_{m}$ are disjoint subsets of $E(M)-X$ such that,

(i) Each $X_{i}$ has either one or two elements;

(ii) $\lambda\left(X \cup X_{1} \cup \cdots \cup X_{i}\right) \leq 4$, for all $i \in[m]$; and

(iii) The sequence $\left(X_{i}\right)_{i=1}^{m}$ has maximal length with respect to properties (i) and (ii).

The full 2-span is a well-defined closure operator on the collection of 4separating sets of $M$ having at least 3 elements. Two exact 4 -separations $(A, B)$ and $(C, D)$ are 2-equivalent if $\left\{\mathrm{fs}_{2}(A), \mathrm{fs}_{2}(B)\right\}=\left\{\mathrm{fs}_{2}(C), \mathrm{fs}_{2}(D)\right\} . \mathrm{A}$ 4-separation $(A, B)$ is sequential if $\mathrm{fs}_{2}(A)=E(M)$ or $\mathrm{fs}_{2}(B)=E(M)$. 


\section{Chapter 3}

\section{Flowers}

In this chapter, we define a notion of equivalence on the $k$-separations of a connectivity system that are non-trivial with respect to a fixed tangle $\mathcal{T}$ of order $k$. We also identify those $k$-separations that are "sequential" with respect to $\mathcal{T}$. We then define $k$-flowers in $\mathcal{T}$ to study how the non-sequential $k$-separations interact. The main goal of the chapter is to prove Theorem 3.6.1, which asserts that, if $\mathcal{T}$ is a tangle of order $k$ that satisfies a certain robustness condition, then every non-sequential $k$-separation conforms with a tight maximal $k$-flower in $\mathcal{T}$. Theorem 3.6.1 is a crucial step towards obtaining the tree structure in Chapter 4 . We conclude the chapter with a section of independent interest, where we obtain a bound on the number of petals in a sequential $k$-separation displayed by a tight $k$-flower in a tangle $\mathcal{T}$.

\subsection{Connectivity systems}

We recall some fundamental notions for connectivity systems that we use in this chapter. For a more detailed treatment of connectivity systems, we refer the reader to Section 2.1.1,

Let $\lambda$ be an integer-valued function on the subsets of a finite set $E$. We call $\lambda$ symmetric if $\lambda(X)=\lambda(E-X)$ for all $X \subseteq E$. We call $\lambda$ submodular if 
$\lambda(X)+\lambda(Y) \geq \lambda(X \cup Y)+\lambda(X \cap Y)$ for all $X, Y \subseteq E$. If $\lambda$ is integer-valued, symmetric, and submodular, then $\lambda$ is called a connectivity function on $E$. If $E$ is a finite set and $\lambda$ is a connectivity function on $E$, then the pair $(E, \lambda)$ is a connectivity system.

Connectivity systems arise naturally from matroids and graphs. Let $M$ be a matroid on ground set $E$ with rank function $r$. For $X \subseteq E$, we let $\lambda_{M}(X)=r(X)+r(E-X)-r(M)+1$. It is straightforward to prove that $\left(E, \lambda_{M}\right)$ is a connectivity system. Let $G$ be a graph with edge set $E$. For $X \subseteq E$, we let $\lambda_{G}(X)$ denote the number of vertices of $G$ that are incident with both an edge of $X$ and an edge of $E-X$. It is also straightforward to prove that $\left(E, \lambda_{G}\right)$ is a connectivity system. Moreover, if $G$ is a connected graph, then $\lambda_{M(G)}(X) \leq \lambda_{G}(X)$.

Let $\lambda$ be a connectivity function on $E$, and let $k$ be a positive integer. A partition $(X, E-X)$ of $E$ is called a $k$-separation of $\lambda$ if $\lambda(X) \leq k$. A subset $X$ of $E$ is said to be $k$-separating in $\lambda$ if $\lambda(X) \leq k$. When the connectivity function $\lambda$ is clear from the context we shall often abbreviate " $k$-separation of $\lambda$ " and " $k$-separating set in $\lambda$ " to " $k$-separation" and " $k$-separating set" respectively. A $k$-separating set $X$, or $k$-separation $(X, E-X)$ is exact if $\lambda(X)=k$.

We note that we consider a $k$-separation $(X, E-X)$ to be an unordered partition of $E$. We also make no assumption on the number of elements in the sets $X$ and $E-X$.

We will make use of the following elementary properties of connectivity functions.

Lemma 3.1.1. [9, Lemma 2.3.] If $\lambda$ is a connectivity function on $E$, then, for all $X, Y \subseteq E$, we have:

(i) $\lambda(X) \geq \lambda(\emptyset)$.

(ii) $\lambda(X)+\lambda(Y) \geq \lambda(X-Y)+\lambda(Y-X)$.

The submodularity of the connectivity function $\lambda$ is frequently used in the following form: 
Lemma 3.1.2. Let $X$ and $Y$ be $k$-separating subsets of $E$.

(i) If $\lambda(X \cap Y) \geq k$, then $X \cup Y$ is $k$-separating.

(ii) If $\lambda(E-(X \cup Y)) \geq k$, then $X \cap Y$ is $k$-separating.

We write by uncrossing to mean "by an application of Lemma 3.1.2'.

\subsection{Tangles}

Let $(E, \lambda)$ be a connectivity system, and let $k$ be a positive integer. Recall that a tangle of order $k$ in $(E, \lambda)$ is a collection $\mathcal{T}$ of subsets of $E$ such that the following properties hold:

(T1) $\lambda(A)<k$ for all $A \in \mathcal{T}$.

(T2) If $(A, E-A)$ is a $(k-1)$-separation, then $\mathcal{T}$ contains $A$ or $E-A$.

(T3) If $A, B, C \in \mathcal{T}$, then $A \cup B \cup C \neq E$.

(T4) $E-\{e\} \notin \mathcal{T}$ for each $e \in E$.

Let $\mathcal{T}$ be a tangle of order $k$ in a connectivity system $(E, \lambda)$. A subset $X$ of $E$ is $\mathcal{T}$-strong if it is not contained in a member of $\mathcal{T}$; otherwise $X$ is $\mathcal{T}$-weak. It is easy to see that supersets of $\mathcal{T}$-strong sets are $\mathcal{T}$-strong, and that subsets of $\mathcal{T}$-weak sets are $\mathcal{T}$-weak. A partition $\left\{X_{1}, \ldots, X_{n}\right\}$ of $E$ is $\mathcal{T}$-strong if $X_{i}$ is a $\mathcal{T}$-strong set for all $i \in[n]$; otherwise $\left\{X_{1}, \ldots, X_{n}\right\}$ is $\mathcal{T}$-weak. In particular, a $k$-separation $(X, E-X)$ of $\lambda$ is $\mathcal{T}$-strong if both $X$ and $E-X$ are $\mathcal{T}$-strong sets; otherwise $(X, E-X)$ is $\mathcal{T}$-weak. We note that if a partition $\{X, E-X\}$ of $E$ is $\mathcal{T}$-strong, then neither $X$ nor $E-X$ is a member of $\mathcal{T}$, so $\lambda(X) \geq k$ by (T2). Thus a $\mathcal{T}$-strong $k$-separation $(X, E-X)$ is exact.

In any unexplained context, if we use the phrase " $\mathcal{T}$-strong $k$-separating set" or "T -strong $k$-separation" without mention of the order of the tangle $\mathcal{T}$, then it will be implicit that $\mathcal{T}$ has order $k$. 


\subsection{Sequential and equivalent $k$-separations}

An essential feature of the tree decompostion of a 3-connected matroid described by Oxley, Semple, and Whittle [23] is that it only guarantees to display, up to equivalence, the non-sequential 3-separations. In this section we fix a tangle $\mathcal{T}$ of order $k$ in a connectivity system $(E, \lambda)$, and we focus on the $k$-separations of $\lambda$ that are $\mathcal{T}$-strong. We define a natural notion of equivalence on the $\mathcal{T}$-strong $k$-separations of a connectivity system with respect to $\mathcal{T}$, and we define what it means for a $\mathcal{T}$-strong $k$-separation to be sequential with respect to $\mathcal{T}$.

Let $\mathcal{T}$ be a tangle of order $k$ in a connectivity system $(E, \lambda) . \mathrm{A} \mathcal{T}$-strong $k$-separating set $X$ is fully closed with respect to $\mathcal{T}$ if $X \cup Y$ is not $k$-separating for every non-empty $\mathcal{T}$-weak set $Y \subseteq E-X$. In particular, we observe that if $X$ is a proper subset of $E$ that is fully closed with respect to $\mathcal{T}$, then $E-X$ is a $\mathcal{T}$-strong set because $X \cup(E-X)$ is $k$-separating by Lemma 3.1.1 (i). We abbreviate "fully closed with respect to $\mathcal{T}$ " to "fully closed" when the tangle $\mathcal{T}$ is clear from the context.

We next develop some results that show every $\mathcal{T}$-strong $k$-separating set is contained in a unique minimal $k$-separating set that is fully closed with respect to $\mathcal{T}$.

Lemma 3.3.1. Let $\mathcal{T}$ be a tangle of order $k$ in a connectivity system $(E, \lambda)$, and let $X$ be a $\mathcal{T}$-strong $k$-separating set in $\lambda$. If $X_{1}$ and $X_{2}$ are fully-closed $k$-separating sets that contain $X$, then there is a fully-closed $k$-separating set $Y$ such that $X \subseteq Y \subseteq X_{1} \cap X_{2}$.

Proof. Let $Y$ be maximal with respect to the properties that $X \subseteq Y \subseteq$ $X_{1} \cap X_{2}$ and $\lambda(Y) \leq k$. Assume towards a contradiction that $Y$ is not fully closed. Then there is some non-empty $\mathcal{T}$-weak set $Z$ contained in $E-Y$ such that $Y \cup Z$ is $k$-separating. If $Z \subseteq X_{1} \cap X_{2}$, then $X \subseteq Y \cup Z \subseteq X_{1} \cap X_{2}$ and $Y \cup Z$ properly contains $Y$; a contradiction of the maximality of $Y$. Therefore, up to switching $X_{1}$ and $X_{2}$, we may assume that $Z$ meets $E-X_{1}$. Now, the partition $\left(Y \cup\left(Z \cap X_{1}\right), E-\left(Y \cup\left(Z \cap X_{1}\right)\right)\right)$ is $\mathcal{T}$-strong because 
$Y \cup\left(Z \cap X_{1}\right)$ contains the $\mathcal{T}$-strong set $X$ while $E-\left(Y \cup\left(Z \cap X_{1}\right)\right)$ contains the $\mathcal{T}$-strong set $E-X_{1}$, so $\lambda\left(Y \cup\left(Z \cap X_{1}\right)\right) \geq k$ by (T2). But $Y \cup\left(Z \cap X_{1}\right)$ is the intersection of the $k$-separating sets $Y \cup Z$ and $X_{1}$, so their union $X_{1} \cup Z$ is $k$-separating by uncrossing; a contradiction because $X_{1}$ is fully closed.

Corollary 3.3.2. Let $\mathcal{T}$ be a tangle of order $k$ in a connectivity system $(E, \lambda)$. Let $X$ be a $\mathcal{T}$-strong $k$-separating set, and let $\mathcal{F}$ be the set of fullyclosed $k$-separating sets that contain $X$. Then $\bigcap_{Y \in \mathcal{F}} Y$ is a fully-closed $k$ separating set that contains $X$.

Proof. It suffices to prove that, for any positive integer $n$, if $X_{1}, X_{2}, \ldots, X_{n} \in$ $\mathcal{F}$, then there is some $Z \in \mathcal{F}$ such that $Z \subseteq \bigcap_{i=1}^{n} X_{i}$. We proceed by induction on $n$. If $n=1$ the result is trivial, so we may assume that $n \geq 2$ and that the result holds for $n-1$. Let $X_{1}, X_{2}, \ldots, X_{n}$ be fully-closed $k$ separating sets that contain $X$. By our inductive hypothesis, there is some $Y \in \mathcal{F}$ such that $Y \subseteq \bigcap_{i=1}^{n-1} X_{i}$. By Lemma 3.3.1, there is some $Z \in \mathcal{F}$ such that $Z \subseteq Y \cap X_{n}$. Hence $Z \subseteq \bigcap_{i=1}^{n} X_{i}$, as required.

Let $\mathcal{T}$ be a tangle of order $k$ in a connectivity system $(E, \lambda)$, and let $X$ be a $\mathcal{T}$-strong $k$-separating set. Then the intersection of all fully-closed $k$-separating sets that contain $X$, which we denote by $\mathrm{fcl}_{\mathcal{T}}(X)$, is called the full closure of $X$ with respect to $\mathcal{T}$. By Corollary 3.3.2, we see that $\mathrm{fcl}_{\mathcal{T}}(X)$ is minimal with respect to being a fully-closed $k$-separating set that contains $X$. We abbreviate "full closure with respect to $\mathcal{T}$ " to "full closure" when the tangle $\mathcal{T}$ is clear from the context.

The next lemma shows that, for a tangle $\mathcal{T}$ of order $k$ in a connectivity system $(E, \lambda)$, the full closure with respect to $\mathcal{T}$ is a closure operator on the set of $\mathcal{T}$-strong $k$-separating sets in $\lambda$.

Lemma 3.3.3. Let $\mathcal{T}$ be a tangle of order $k$ in a connectivity system $(E, \lambda)$. Let $X$ and $Y$ be $\mathcal{T}$-strong $k$-separating sets. Then the following hold:

(i) $X \subseteq \operatorname{fcl}_{\mathcal{T}}(X)$

(ii) If $X \subseteq Y$, then $\operatorname{fcl}_{\mathcal{T}}(X) \subseteq \operatorname{fcl}_{\mathcal{T}}(Y)$. 
(iii) $\operatorname{fcl}_{\mathcal{T}}\left(\operatorname{fcl}_{\mathcal{T}}(X)\right)=\mathrm{fcl}_{\mathcal{T}}(X)$.

Proof. It follows from the definition of $\operatorname{fcl}_{\mathcal{T}}(X)$ that $X \subseteq \mathrm{fcl}_{\mathcal{T}}(X)$. Thus (i) holds. For (ii), suppose that $X \subseteq Y$. Then $\mathrm{fcl}_{\mathcal{T}}(Y)$ is a fully-closed $k$ separating set that contains $X$, so $\operatorname{fcl}_{\mathcal{T}}(X) \subseteq \mathrm{fcl}_{\mathcal{T}}(Y)$. For (iii), it follows from (i) that $\mathrm{fcl}_{\mathcal{T}}(X) \subseteq \mathrm{fcl}_{\mathcal{T}}\left(\mathrm{fcl}_{\mathcal{T}}(X)\right)$. On the other hand, $\mathrm{fcl}_{\mathcal{T}}(X)$ is a fullyclosed $k$-separating set that contains $\mathrm{fcl}_{\mathcal{T}}(X)$, so $\mathrm{fcl}_{\mathcal{T}}\left(\mathrm{fcl}_{\mathcal{T}}(X)\right) \subseteq \mathrm{fcl}_{\mathcal{T}}(X)$.

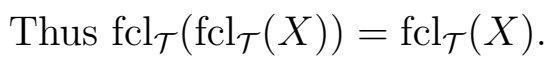

Let $\mathcal{T}$ be a tangle of order $k$ in a connectivity system $(E, \lambda)$. We use the full closure with respect to $\mathcal{T}$ to define a binary relation called $\mathcal{T}$-equivalence on the set of $\mathcal{T}$-strong $k$-separating sets in $\lambda$. Let $X$ and $Y$ be $\mathcal{T}$-strong $k$-separating sets in $\lambda$. Then $X$ is $\mathcal{T}$-equivalent to $Y$ if $\operatorname{fcl}_{\mathcal{T}}(X)=\operatorname{fcl}_{\mathcal{T}}(Y)$. In what follows, we suppress the tangle $\mathcal{T}$ and say that $X$ is "equivalent" to $Y$ when the tangle $\mathcal{T}$ is clear from the context.

We note that if $M$ is a 3-connected matroid on ground set $E$, and $\mathcal{T}$ is the unique tangle of order three in the connectivity system $\left(E, \lambda_{M}\right)$, then the relation $\mathcal{T}$-equivalent for exact 3 -separating sets is precisely the relation "equivalent" defined by Oxley, Semple, and Whittle in [23].

We omit the elementary proof of the next result.

Lemma 3.3.4. Let $\mathcal{T}$ be a tangle of order $k$ in a connectivity system $(E, \lambda)$. Then the binary relation $\mathcal{T}$-equivalent is an equivalence relation on the set of $\mathcal{T}$-strong $k$-separating sets in $\lambda$.

Recall that Oxley, Semple, and Whittle [23] used a full closure operator to define equivalence of 3-separations in 3-connected matroids. Let $M$ be a 3 -connected matroid, and let $X$ be a 3 -separating set in $M$ having at least 2 elements. It is easily seen that $\mathrm{fcl}(X)$ is a maximal set containing $X$ for which the members of $\mathrm{fcl}(X)-X$ can be ordered $\left(x_{1}, \ldots, x_{n}\right)$ such that, for all $i \in[n]$, the set $X \cup\left\{x_{1}, \ldots, x_{i}\right\}$ is 3 -separating. We can restate this characterisation of the full closure in a more tangle-theoretic way as follows. The full closure of $X$ is a maximal set of the form $X \cup\left\{x_{1}\right\} \cup \cdots \cup\left\{x_{n}\right\}$, 
where $\left(\left\{x_{1}\right\}, \ldots,\left\{x_{n}\right\}\right)$ is a sequence of $\mathcal{T}$-weak sets such that, for all $i \in[n]$, the set $X \cup\left\{x_{1}\right\} \cup \cdots \cup\left\{x_{i}\right\}$ is 3-separating. For a tangle $\mathcal{T}$ of order $k$ in a connectivity system $(E, \lambda)$ and a $\mathcal{T}$-strong $k$-separating set $X$, we now work towards a similar characterisation of the full closure of $X$ with respect to $\mathcal{T}$.

Let $\mathcal{T}$ be a tangle of order $k$ in a connectivity system $(E, \lambda)$, and let $X$ be a $\mathcal{T}$-strong $k$-separating set in $\lambda$. A partial $k$-sequence for $X$ is a sequence $\left(X_{i}\right)_{i=1}^{m}$ of pairwise disjoint, non-empty $\mathcal{T}$-weak subsets of $E-X$ such that $X \cup\left(\bigcup_{i=1}^{j} X_{i}\right)$ is $k$-separating for all $j \in[m]$.

Lemma 3.3.5. Let $\mathcal{T}$ be a tangle of order $k$ in a connectivity system $(E, \lambda)$, and let $X$ be a $\mathcal{T}$-strong $k$-separating set in $\lambda$. If $\left(X_{i}\right)_{i=1}^{m}$ is a partial $k$ sequence for $X$, then $X \cup\left(\bigcup_{i=1}^{m} X_{i}\right) \subseteq \operatorname{fcl}_{\mathcal{T}}(X)$.

Proof. Suppose that $\left(X_{i}\right)_{i=1}^{m}$ is a partial $k$-sequence for $X$ such that $X \cup$ $\left(\bigcup_{i=1}^{m} X_{i}\right)$ is not contained in $\operatorname{fcl}_{\mathcal{T}}(X)$. Then $\operatorname{fcl}_{\mathcal{T}}(X) \neq E$, so $E-\operatorname{fcl}_{\mathcal{T}}(X)$ is a $\mathcal{T}$-strong set. Let $j \in[m]$ be the smallest index such that $X_{j}$ is not contained in $\mathrm{fcl}_{\mathcal{T}}(X)$. Now, the partition

$$
\left(\left(X \cup\left(\bigcup_{i=1}^{j} X_{i}\right)\right) \cap \mathrm{fcl}_{\mathcal{T}}(X), E-\left(\left(X \cup\left(\bigcup_{i=1}^{j} X_{i}\right)\right) \cap \mathrm{fcl}_{\mathcal{T}}(X)\right)\right)
$$

of $E$ is $\mathcal{T}$-strong because $\left(X \cup\left(\bigcup_{i=1}^{j} X_{i}\right)\right) \cap \mathrm{fcl}_{\mathcal{T}}(X)$ contains the $\mathcal{T}$-strong set $X$ while $E-\left(\left(X \cup\left(\bigcup_{i=1}^{j} X_{i}\right)\right) \cap \mathrm{fcl}_{\mathcal{T}}(X)\right)$ contains the $\mathcal{T}$-strong set $E-\mathrm{fcl}_{\mathcal{T}}(X)$. Thus $\lambda\left(\left(X \cup\left(\bigcup_{i=1}^{j} X_{i}\right)\right) \cap \mathrm{fcl}_{\mathcal{T}}(X)\right) \geq k$ by (T2). Then uncrossing the $k$ separating sets $X \cup\left(\bigcup_{i=1}^{j} X_{i}\right)$ and $\operatorname{fcl}_{\mathcal{T}}(X)$ we see that $\mathrm{fcl}_{\mathcal{T}}(X) \cup X_{j}$ is $k$ separating; a contradiction because $\mathrm{fcl}_{\mathcal{T}}(X)$ is fully closed.

Since the full closure with respect to $\mathcal{T}$ is a closure operator on the set of $\mathcal{T}$-strong $k$-separating sets, we have the following immediate corollary of Lemma 3.3.5. We omit the straightforward proof.

Corollary 3.3.6. Let $\mathcal{T}$ be a tangle of order $k$ in a connectivity system $(E, \lambda)$, and let $X$ be a $\mathcal{T}$-strong $k$-separating set. If $\left(X_{i}\right)_{i=1}^{m}$ is a partial $k$ sequence for $X$, then $\mathrm{fcl}_{\mathcal{T}}\left(X \cup\left(\bigcup_{i=1}^{m} X_{i}\right)\right)=\mathrm{fcl}_{\mathcal{T}}(X)$. 
Let $\mathcal{T}$ be a tangle of order $k$ in a connectivity system $(E, \lambda)$, and let $X$ be a $\mathcal{T}$-strong $k$-separating set in $\lambda$. Let

$$
P=\left\{X \cup\left(\bigcup_{i=1}^{m} X_{i}\right) \mid\left(X_{i}\right)_{i=1}^{m} \text { is a partial } k \text {-sequence for } X\right\} .
$$

Then it is easy to see that $(P, \subseteq)$ is a poset. A partial $k$-sequence $\left(X_{i}\right)_{i=1}^{m}$ for $X$ is said to be maximal if $X \cup\left(\bigcup_{i=1}^{m} X_{i}\right)$ is maximal in the poset $(P, \subseteq)$.

Lemma 3.3.7. Let $\mathcal{T}$ be a tangle of order $k$ in a connectivity system $(E, \lambda)$. Let $X$ be a $\mathcal{T}$-strong $k$-separating set, and let $\left(X_{i}\right)_{i=1}^{m}$ be a partial $k$-sequence for $X$. Then $\mathrm{fcl}_{\mathcal{T}}(X)=X \cup\left(\bigcup_{i=1}^{m} X_{i}\right)$ if and only if $\left(X_{i}\right)_{i=1}^{m}$ is maximal.

Proof. Assume that $\mathrm{fcl}_{\mathcal{T}}(X)=X \cup\left(\bigcup_{i=1}^{m} X_{i}\right)$. Then $\left(X_{i}\right)_{i=1}^{m}$ is maximal by Lemma 3.3.5. Conversely, assume that $\left(X_{i}\right)_{i=1}^{m}$ is maximal. Then it follows from Lemma 3.3 .5 that $X \cup\left(\bigcup_{i=1}^{m} X_{i}\right) \subseteq \mathrm{fcl}_{\mathcal{T}}(X)$. We claim that $X \cup\left(\bigcup_{i=1}^{m} X_{i}\right)$ is fully closed. Suppose that $X \cup\left(\bigcup_{i=1}^{m} X_{i}\right)$ is not fully closed. Then there is a non-empty $\mathcal{T}$-weak subset $X_{m+1}$ of $E-\left(X \cup\left(\bigcup_{i=1}^{m} X_{i}\right)\right)$ such that $X \cup\left(\bigcup_{i=1}^{m+1} X_{i}\right)$ is $k$-separating. Hence $\left(X_{i}\right)_{i=1}^{m+1}$ is a partial $k$-sequence, and $X \cup\left(\bigcup_{i=1}^{m} X_{i}\right) \subsetneq X \cup\left(\bigcup_{i=1}^{m+1} X_{i}\right)$; a contradiction because $\left(X_{i}\right)_{i=1}^{m}$ is maximal. Thus $X \cup\left(\bigcup_{i=1}^{m} X_{i}\right)$ is a fully-closed $k$-separating set that contains $X$, so $\mathrm{fcl}_{\mathcal{T}}(X) \subseteq X \cup\left(\bigcup_{i=1}^{m} X_{i}\right)$. Therefore $\mathrm{fcl}_{\mathcal{T}}(X)=X \cup\left(\bigcup_{i=1}^{m} X_{i}\right)$, as required.

We shall abbreviate "maximal partial $k$-sequence" to " $k$-sequence".

Let $\mathcal{T}$ be a tangle of order $k$ in a connectivity system $(E, \lambda)$. We extend the relation $\mathcal{T}$-equivalent to the set of $\mathcal{T}$-strong $k$-separations of $\lambda$ in the natural way. Let $(X, Y)$ and $\left(X^{\prime}, Y^{\prime}\right)$ be $\mathcal{T}$-strong $k$-separations of $\lambda$. Then $(X, Y)$ is $\mathcal{T}$-equivalent to $\left(X^{\prime}, Y^{\prime}\right)$ if $\left\{\operatorname{fcl}_{\mathcal{T}}(X), \operatorname{fcl}_{\mathcal{T}}(Y)\right\}=\left\{\operatorname{fcl}_{\mathcal{T}}\left(X^{\prime}\right), \operatorname{fcl}_{\mathcal{T}}\left(Y^{\prime}\right)\right\}$. We note that, when the tangle $\mathcal{T}$ is clear from the context, we shall abbreviate "T-equivalent" to "equivalent".

The following lemma is straightforward to prove. We omit the details.

Lemma 3.3.8. Let $\mathcal{T}$ be a tangle of order $k$ in a connectivity system $(E, \lambda)$. Then the binary relation $\mathcal{T}$-equivalent is an equivalence relation on the set of $\mathcal{T}$-strong $k$-separations of $\lambda$. 
We note that if $M$ is a 3-connected matroid on ground set $E$, and $\mathcal{T}$ is the unique tangle of order three in the connectivity system $\left(E, \lambda_{M}\right)$, then the relation $\mathcal{T}$-equivalent for exact 3 -separating partitions is precisely the relation "equivalent" defined by Oxley, Semple, and Whittle [23]. Similarly, if $M$ is a 4-connected matroid on ground set $E$, and $\mathcal{T}$ is the unique tangle of order four in the connectivity system $\left(E, \lambda_{M}\right)$, then the relation $\mathcal{T}$-equivalent for exact 4-separating partitions is precisely the relation "2-equivalent" defined by Aikin [1].

Let $\mathcal{T}$ be a tangle of order $k$ in a connectivity system $(E, \lambda)$, and let $X$ be a $\mathcal{T}$-strong $k$-separating set in $\lambda$. We say that $E-X$ is $\mathcal{T}$-sequential if $\operatorname{fcl}_{\mathcal{T}}(X)=E$. A $k$-separation $(X, Y)$ is $\mathcal{T}$-sequential if $X$ or $Y$ is a $\mathcal{T}$ sequential $k$-separating set. When the tangle $\mathcal{T}$ is clear from the context, we shall use "sequential" and "non-sequential" instead of " $\mathcal{T}$-sequential" and "not $\mathcal{T}$-sequential" respectively.

We note that, for a tangle $\mathcal{T}$ of order $k$ in a connectivity system $(E, \lambda)$, if $X$ is a $\mathcal{T}$-strong $k$-separating set, but $(X, E-X)$ is not a $\mathcal{T}$-strong $k$-separation, then it is easy to see that $(X, E-X)$ is a $\mathcal{T}$-sequential $k$-separation. Thus every non-sequential $k$-separation is a $\mathcal{T}$-strong $k$ separation.

The remainder of this section is devoted to developing some useful lemmas about $k$-separations of a connectivity function $\lambda$ that are equivalent with respect to a fixed tangle $\mathcal{T}$ of order $k$.

Lemma 3.3.9. Let $\mathcal{T}$ be a tangle of order $k$ in a connectivity system $(E, \lambda)$. Let $(R, G)$ be a $\mathcal{T}$-strong $k$-separation of $\lambda$, and let $A \subseteq G$ be a non-empty $\mathcal{T}$-weak set. If $R \cup A$ is $k$-separating and $G-A$ is $\mathcal{T}$-strong, then $(R, G)$ is $\mathcal{T}$-equivalent to $(R \cup A, G-A)$.

Proof. Suppose that $R \cup A$ is $k$-separating and that $G-A$ is $\mathcal{T}$-strong. Then the single-term sequence $(A)$ is a partial $k$-sequence for both $R$ and $G-A$, so $\mathrm{fcl}_{\mathcal{T}}(R)=\mathrm{fcl}_{\mathcal{T}}(R \cup A)$ and $\mathrm{fcl}_{\mathcal{T}}(G-A)=\mathrm{fcl}_{\mathcal{T}}(G)$ by Corollary 3.3.6.

The following lemma is used frequently. 
Lemma 3.3.10. Let $\mathcal{T}$ be a tangle of order $k$ in a connectivity system $(E, \lambda)$. Let $(R, G)$ be a non-sequential k-separation of $\lambda$, and let $A \subseteq G$ be a nonempty $\mathcal{T}$-weak set. If $R \cup A$ is a k-separating set, then $(R, G)$ is $\mathcal{T}$-equivalent to $(R \cup A, G-A)$.

Proof. Assume that $R \cup A$ is a $k$-separating set. By Lemma 3.3.9, it suffices to show that $G-A$ is $\mathcal{T}$-strong. Seeking a contradiction, suppose that $G-A$ is $\mathcal{T}$-weak. Then the sequence $(A, G-A)$ is a partial $k$-sequence for $R$, so $E=\mathrm{fcl}_{\mathcal{T}}(R)$ by Corollary 3.3.6; a contradiction because $(R, G)$ is non-sequential. Thus $G-A$ is $\mathcal{T}$-strong, so the result follows from Lemma 3.3 .9 .

The following lemma provides an economical test of equivalence for nonsequential $k$-separations. It extends a result of Oxley, Semple, and Whittle [23. Lemma 3.3].

Lemma 3.3.11. Let $\mathcal{T}$ be a tangle of order $k$ in a connectivity system $(E, \lambda)$, and let $(A, B)$ and $(C, D)$ be two non-sequential $k$-separations of $\lambda$. Then $(A, B)$ is equivalent to $(C, D)$ if and only if either $\operatorname{fcl}_{\mathcal{T}}(A)=\operatorname{fcl}_{\mathcal{T}}(C)$ or $\mathrm{fcl}_{\mathcal{T}}(A)=\mathrm{fcl}_{\mathcal{T}}(D)$.

Proof. In one direction the lemma is trivial. For the other direction, assume that $\operatorname{fcl}_{\mathcal{T}}(A)=\mathrm{fcl}_{\mathcal{T}}(C)=Y$. Set $X=E-Y$. Then $X$ is a $\mathcal{T}$-strong $k$ separating set, since $(A, B)$ is non-sequential. Let $\left(A_{i}\right)_{i=1}^{m}$ be a $k$-sequence for $A$ and $\left(C_{i}\right)_{i=1}^{n}$ be a $k$-sequence for $C$. Then it follows from Lemma 3.3.7 that $A \cup\left(\bigcup_{i=1}^{m} A_{i}\right)=\mathrm{fcl}_{\mathcal{T}}(A)$ and $C \cup\left(\bigcup_{i=1}^{n} C_{i}\right)=\mathrm{fcl}_{\mathcal{T}}(C)$. Then $\left(A_{m-i+1}\right)_{i=1}^{m}$ and $\left(C_{n-i+1}\right)_{i=1}^{n}$ are partial $k$-sequences for $X$, so $B=X \cup\left(\bigcup_{i=1}^{m} A_{m-i+1}\right) \subseteq$ $\mathrm{fcl}_{\mathcal{T}}(X)$ and $D=X \cup\left(\bigcup_{i=1}^{n} C_{n-i+1}\right) \subseteq \mathrm{fcl}_{\mathcal{T}}(X)$ by Lemma 3.3.5. By Lemma 3.3.3, and since $X$ is a subset of both $B$ and $D$, we have $\operatorname{fcl}_{\mathcal{T}}(B)=\operatorname{fcl}_{\mathcal{T}}(X)=$ $\mathrm{fcl}_{\mathcal{T}}(D)$, so $(A, B)$ is indeed equivalent to $(C, D)$.

Lemma 3.3.12. Let $\mathcal{T}$ be a tangle of order $k$ in a connectivity system $(E, \lambda)$. If $(R, G)$ is a non-sequential $k$-separation of $\lambda$, then $\left(\operatorname{fcl}_{\mathcal{T}}(R), E-\mathrm{fcl}_{\mathcal{T}}(R)\right)$ is a non-sequential $k$-separation of $\lambda$ that is equivalent to $(R, G)$. 
Proof. Suppose that $(R, G)$ is a non-sequential $k$-separation of $\lambda$. Then $E-\mathrm{fcl}_{\mathcal{T}}(R)$ is a $\mathcal{T}$-strong $k$-separating set. Thus $\left(\mathrm{fcl}_{\mathcal{T}}(R), E-\mathrm{fcl}_{\mathcal{T}}(R)\right)$ is a $\mathcal{T}$-strong $k$-separation. Moreover, it follows from Lemma 3.3 .3 that $\mathrm{fcl}_{\mathcal{T}}\left(E-\mathrm{fcl}_{\mathcal{T}}(R)\right) \subseteq \mathrm{fcl}_{\mathcal{T}}(G) \neq E$ and $\mathrm{fcl}_{\mathcal{T}}\left(\operatorname{fcl}_{\mathcal{T}}(R)\right)=\mathrm{fcl}_{\mathcal{T}}(R) \neq E$, so $(E-$ $\left.\mathrm{fcl}_{\mathcal{T}}(R), \mathrm{fcl}_{\mathcal{T}}(R)\right)$ is non-sequential. Then $(R, G)$ and $\left(\mathrm{fcl}_{\mathcal{T}}(R), E-\mathrm{fcl}_{\mathcal{T}}(R)\right)$ are non-sequential $k$-separations, and $\mathrm{fcl}_{\mathcal{T}}\left(\mathrm{fcl}_{\mathcal{T}}(R)\right)=\mathrm{fcl}_{\mathcal{T}}(R)$, so $(R, G)$ is $\mathcal{T}$-equivalent to $\left(\mathrm{fcl}_{\mathcal{T}}(R), E-\mathrm{fcl}_{\mathcal{T}}(R)\right)$ by Lemma 3.3.11.

Lemma 3.3.13. Let $\mathcal{T}$ be a tangle of order $k$ in a connectivity system $(E, \lambda)$, and let $\mathcal{K}$ be an equivalence class of non-sequential $k$-separations of $\lambda$. If $\left(X_{1}, Y_{1}\right),\left(X_{2}, Y_{2}\right) \in \mathcal{K}$ and $\mathrm{fcl}_{\mathcal{T}}\left(X_{1}\right)=\mathrm{fcl}_{\mathcal{T}}\left(X_{2}\right)$, then there is a non-sequential k-separation $\left(X_{3}, Y_{3}\right) \in \mathcal{K}$ such that $X_{3} \subseteq X_{1} \cap X_{2}$.

Proof. Assume that $\left(X_{1}, Y_{1}\right),\left(X_{2}, Y_{2}\right) \in \mathcal{K}$ and $\operatorname{fcl}_{\mathcal{T}}\left(X_{1}\right)=\operatorname{fcl}_{\mathcal{T}}\left(X_{2}\right)$. Thus $\operatorname{fcl}_{\mathcal{T}}\left(Y_{1}\right)=\mathrm{fcl}_{\mathcal{T}}\left(Y_{2}\right)$. Then $E-\mathrm{fcl}_{\mathcal{T}}\left(Y_{1}\right) \subseteq X_{1} \cap X_{2}$, and $(E-$ $\left.\mathrm{fcl}_{\mathcal{T}}\left(Y_{1}\right), \mathrm{fcl}_{\mathcal{T}}\left(Y_{1}\right)\right) \in \mathcal{K}$ by Lemma 3.3.12,

Lemma 3.3.14. Let $\mathcal{T}$ be a tangle of order $k$ in a connectivity system $(E, \lambda)$, and let $(R, G)$ be a non-sequential $k$-separation of $\lambda$. If $X$ is a $k$-separating set such that $E-\operatorname{fcl}_{\mathcal{T}}(G) \subseteq X \subseteq R$, then $(X, E-X)$ is $\mathcal{T}$-equivalent to $(R, G)$.

Proof. Suppose that $X$ is a $k$-separating set such that $E-\operatorname{fcl}_{\mathcal{T}}(G) \subseteq X \subseteq$ $R$. Then $\operatorname{fcl}_{\mathcal{T}}\left(E-\mathrm{fcl}_{\mathcal{T}}(G)\right) \subseteq \operatorname{fcl}_{\mathcal{T}}(X) \subseteq \mathrm{fcl}_{\mathcal{T}}(R)$ by Lemma 3.3.3, and so $\mathrm{fcl}_{\mathcal{T}}(X)=\mathrm{fcl}_{\mathcal{T}}(R)$ by Lemma 3.3 .12 . Then from the fact that $E-\mathrm{fcl}_{\mathcal{T}}(G) \subseteq$ $X \subseteq R$ it follows that $G \subseteq E-X \subseteq \mathrm{fcl}_{\mathcal{T}}(G)$, $\operatorname{so~fcl}_{\mathcal{T}}(G)=\operatorname{fcl}_{\mathcal{T}}(E-X)$ by Lemma 3.3.3. Thus $(X, E-X)$ is $\mathcal{T}$-equivalent to $(R, G)$.

Lemma 3.3.15. Let $\mathcal{T}$ be a tangle of order $k$ in a connectivity system $(E, \lambda)$, and let $(X, Y)$ and $(R, G)$ be non-sequential $k$-separations of $\lambda$. If $R \subseteq X$, then there is a non-sequential $k$-separation $\left(R^{\prime}, G^{\prime}\right)$ that is $\mathcal{T}$-equivalent to $(R, G)$ such that $R^{\prime} \subseteq\left(E-\mathrm{fcl}_{\mathcal{T}}(Y)\right)$.

Proof. Assume that $R \subseteq X$. Then $Y \subseteq G$, so $\operatorname{fcl}_{\mathcal{T}}(Y) \subseteq \operatorname{fcl}_{\mathcal{T}}(G)$ by Lemma 3.3.3. Thus $E-\mathrm{fcl}_{\mathcal{T}}(G) \subseteq E-\mathrm{fcl}_{\mathcal{T}}(Y)$, and the $k$-separation $\left(E-\mathrm{fcl}_{\mathcal{T}}(G), \mathrm{fcl}_{\mathcal{T}}(G)\right)$ is $\mathcal{T}$-equivalent to $(R, G)$ by lemma 3.3 .12 . 


\subsection{Flowers in a tangle}

Let $\mathcal{T}$ be a tangle of order $k$ in a connectivity system $(E, \lambda)$, and let $n$ be a positive integer. A $\mathcal{T}$-strong partition $\left(P_{1}, \ldots, P_{n}\right)$ of $E$ is a $k$-flower in $\mathcal{T}$ with petals $P_{1}, \ldots, P_{n}$ if, for all $i$, both $P_{i}$ and $P_{i} \cup P_{i+1}$ are $k$-separating sets, where all subscripts are interpreted modulo $n$.

We note two features of this definition. First, recall that Aikin and Oxley in [2] defined a $k$-flower in connectivity function $\lambda$ on $E$ to be a partition $\left(P_{1}, \ldots, P_{n}\right)$ of $E$ such that, for all $i$, both $P_{i}$ and $P_{i} \cup P_{i+1}$ are exactly $k$-separating, where all subscripts are interpreted modulo $n$. Thus, every $k$ flower in $\mathcal{T}$ is also a $k$-flower in $\lambda$. However, in this thesis the main purpose of a $k$-flower in $\mathcal{T}$ is to "display" a collection of inequivalent non-sequential $k$-separations, and so, given our definition of equivalence of $k$-separations, it is natural to require the petals of a $k$-flower in $\mathcal{T}$ to be $\mathcal{T}$-strong sets. Second, we note that, if $M$ is a 3 -connected matroid on ground set $E$, and $\mathcal{T}$ is the unique tangle of order three in $\left(E, \lambda_{M}\right)$, then a 3 -flower in $\mathcal{T}$ is a flower in $M$ as defined by Oxley, Semple, and Whittle [23.

We next define some of the fundamental notions for $k$-flowers in $\mathcal{T}$. Most of these are natural extensions of the analogous notions for flowers in 3connected matroids given in [23].

Let $\mathcal{T}$ be a tangle of order $k$ in a connectivity system $(E, \lambda)$, and let $\Phi=\left(P_{1}, \ldots, P_{n}\right)$ be a $k$-flower in $\mathcal{T}$. A $k$-separating set $X$ or $k$-separation $(X, E-X)$ is said to be displayed by $\Phi$ if $X$ is a union of petals of $\Phi$. For a non-empty subset $I$ of $[n]$, we write $P_{I}$ for $\bigcup_{i \in I} P_{i}$. A $k$-flower $\Phi=$ $\left(P_{1}, \ldots, P_{n}\right)$ in $\mathcal{T}$ is called a $k$-anemone if $P_{I}$ is $k$-separating for any nonempty subset $I$ of $[n]$, and a $k$-daisy if $P_{I}$ is $k$-separating for precisely those non-empty subsets $I$ of $[n]$ whose members form a consecutive set in the cyclic order $(1, \ldots, n)$. As every $k$-flower in $\mathcal{T}$ is a $k$-flower in the connectivity function $\lambda$, we have the following immediate consequence of [2, Theorem 1.1].

Corollary 3.4.1. Every $k$-flower in $\mathcal{T}$ is either a $k$-anemone or a $k$-daisy.

Recall that a quasi-order is a reflexive and transitive relation. Let $\mathcal{T}$ be a 
tangle of order $k$ in a connectivity system $(E, \lambda)$. We define a relation $\preccurlyeq$ on the set of $k$-flowers in $\mathcal{T}$ as follows. Let $\Phi_{1}$ and $\Phi_{2}$ be $k$-flowers in $\mathcal{T}$. We say that $\Phi_{1} \preccurlyeq \Phi_{2}$ if, for every non-sequential $k$-separation displayed by $\Phi_{1}$, there is some $\mathcal{T}$-equivalent $k$-separation displayed by $\Phi_{2}$. It is straightforward to verify that the relation $\preccurlyeq$ is a quasi-order on the set of $k$-flowers in $\mathcal{T}$. If $\Phi_{1} \preccurlyeq \Phi_{2}$ and $\Phi_{2} \preccurlyeq \Phi_{1}$, we say that $\Phi_{1}$ and $\Phi_{2}$ are $\mathcal{T}$-equivalent $k$-flowers. Thus, $\mathcal{T}$-equivalent $k$-flowers display, up to $\mathcal{T}$-equivalence of $k$-separations, exactly the same non-sequential $k$-separations. As with the other notions of equivalence that we have developed, when the tangle $\mathcal{T}$ is clear from the context, we shall abbreviate "T -equivalent" to "equivalent".

Let $\Phi=\left(P_{1}, \ldots, P_{n}\right)$ be a $k$-flower in $\mathcal{T}$. If $\Phi$ is a $k$-anemone and $\sigma$ is an arbitrary permutation of the set $[n]$, then it is easy to see that $\Phi^{\prime}=$ $\left(P_{\sigma(1)}, \ldots, P_{\sigma(n)}\right)$ is a $k$-flower in $\mathcal{T}$ that is equivalent to $\Phi$. Similarly, if $\Phi=\left(P_{1}, \ldots, P_{n}\right)$ is a $k$-daisy and $\sigma$ is a permutation of the set $[n]$ that corresponds to a symmetry of a regular $n$-gon, then it is easy to see that $\Phi^{\prime}=\left(P_{\sigma(1)}, \ldots, P_{\sigma(n)}\right)$ is a $k$-flower in $\mathcal{T}$ that is equivalent to $\Phi$. We say that $\Phi$ and $\Phi^{\prime}$ are equal up to labels. We will often use the phrase "up to labels" to mean "by an appropriate permutation of the petals".

We now describe a fundamental method of obtaining new $k$-flowers in $\mathcal{T}$ from old. Let $\Phi=\left(P_{1}, P_{2}, \ldots, P_{n}\right)$ be a $k$-flower in $\mathcal{T}$. Then the ordered partition $\Phi^{\prime}=\left(P_{1}^{\prime}, P_{2}^{\prime}, \ldots, P_{m}^{\prime}\right)$ is a concatenation of $\Phi$ if there are indices $0=j_{0}<j_{1}<j_{2}<\cdots<j_{m}=m$ such that $P_{i}^{\prime}=P_{j_{i-1}+1} \cup \cdots \cup P_{j_{i}}$ for all $i \in[m]$. If $\Phi^{\prime}$ is a concatenation of $\Phi$, then $\Phi$ refines $\Phi^{\prime}$.

We will use the following result later without reference. We omit the details of the straightforward proof.

Lemma 3.4.2. Let $\mathcal{T}$ be a tangle of order $k$ in a connectivity system $(E, \lambda)$. If $\Phi=\left(P_{1}, P_{2}, \ldots, P_{n}\right)$ is a $k$-flower in $\mathcal{T}$, and $\Phi^{\prime}=\left(P_{1}^{\prime}, P_{2}^{\prime}, \ldots, P_{m}^{\prime}\right)$ is a concatenation of $\Phi$, then $\Phi^{\prime}$ is a $k$-flower in $\mathcal{T}$.

The following is an economical way to show that a $\mathcal{T}$-strong partition of $E$ is a $k$-flower in $\mathcal{T}$. 
Lemma 3.4.3. Let $\mathcal{T}$ be a tangle of order $k$ in a connectivity system $(E, \lambda)$. Let $n \geq 4$, and let $\Phi=\left(P_{1}, \ldots, P_{n}\right)$ be a $\mathcal{T}$-strong partition of $E$. If $P_{i} \cup P_{i+1}$ is $k$-separating for each $i \in[n-1]$, then $\Phi$ is a $k$-flower in $\mathcal{T}$.

Proof. Suppose that $P_{i} \cup P_{i+1}$ is $k$-separating for each $i \in[n-1]$. We first show that $P_{n} \cup P_{1}$ is $k$-separating. If $n=4$, then $P_{n} \cup P_{1}$ is $k$-separating because $P_{2} \cup P_{3}$ is $k$-separating and $\lambda$ is symmetric. If $n>4$, the sets $P_{2} \cup P_{3}$ and $P_{3} \cup P_{4}$ are $k$-separating, and their intersection is the set $P_{3}$. Since the partition $\left(P_{3}, E-P_{3}\right)$ is $\mathcal{T}$-strong, we deduce that $\lambda\left(P_{3}\right) \geq k$ by (T2). Then $P_{2} \cup P_{3} \cup P_{4}$ is $k$-separating by uncrossing $P_{2} \cup P_{3}$ and $P_{3} \cup P_{4}$. By repeated uncrossings, we deduce that $P_{2} \cup \cdots \cup P_{n-1}$ is $k$-separating, so the complement $E-\left(P_{2} \cup \cdots \cup P_{n-1}\right)=P_{n} \cup P_{1}$ is $k$-separating. Thus the union of any two members of $\Phi$ that are consecutive in the cyclic order is $k$ separating. Furthermore, for each $i \in[n]$, the set $P_{i}$ is the intersection of the $k$-separating sets $P_{i-1} \cup P_{i}$ and $P_{i} \cup P_{i+1}$, where all subscripts are interpreted modulo $n$. Since $n \geq 4$, the partition $\left(P_{i-1} \cup P_{i} \cup P_{i+1}, E-\left(P_{i-1} \cup P_{i} \cup P_{i+1}\right)\right)$ is $\mathcal{T}$-strong, so $\lambda\left(P_{i-1} \cup P_{i} \cup P_{i+1}\right) \geq k$ by (T2). Thus the set $P_{i}$ is $k$-separating by uncrossing $P_{i-1} \cup P_{i}$ and $P_{i} \cup P_{i+1}$. Therefore $\Phi$ is a $k$-flower in $\mathcal{T}$.

The order of a $k$-flower $\Phi$ in $\mathcal{T}$ is the minimum number of petals in a $k$-flower equivalent to $\Phi$. Up to equivalence of $k$-separations, a $k$-flower of order one displays no non-sequential $k$-separations, a $k$-flower of order two displays exactly one non-sequential $k$-separation, and a $k$-flower of order at least three displays at least two non-sequential $k$-separations. A $k$-flower is tight if it is not equivalent to a $k$-flower with fewer petals.

Let $n \geq 2$, and let $\Phi=\left(P_{1}, \ldots, P_{n}\right)$ be a $k$-flower in $\mathcal{T}$. The petal $P_{i}$ of $\Phi$ is $\mathcal{T}$-loose if $P_{i} \subseteq \operatorname{fcl}_{\mathcal{T}}\left(P_{\sigma(i+1)}\right)$ for some permutation $\sigma$ of $[n]$ such that $\sigma(i)=i$ and $\left(P_{\sigma(1)}, \ldots, P_{\sigma(n)}\right)$ is equal to $\Phi$ up to labels. The next result shows that $k$-flowers in $\mathcal{T}$ that have $\mathcal{T}$-loose petals are not tight.

Lemma 3.4.4. Let $\mathcal{T}$ be a tangle of order $k$ in a connectivity system $(E, \lambda)$. Let $n \geq 2$, and let $\Phi=\left(P_{1}, \ldots, P_{n}\right)$ be a $k$-flower in $\mathcal{T}$. If $P_{1} \subseteq \operatorname{fcl}_{\mathcal{T}}\left(P_{2}\right)$, then the concatenation $\Phi^{\prime}=\left(P_{1} \cup P_{2}, P_{3}, \ldots, P_{n}\right)$ of $\Phi$ is $\mathcal{T}$-equivalent to $\Phi$. 
Proof. Assume that $P_{1} \subseteq \mathrm{fcl}_{\mathcal{T}}\left(P_{2}\right)$, and let $\Phi^{\prime}=\left(P_{1} \cup P_{2}, P_{3}, \ldots, P_{n}\right)$. Since $\Phi$ refines $\Phi^{\prime}$, it is immediate that $\Phi^{\prime} \preccurlyeq \Phi$. Assume that $(R, G)$ is a nonsequential $k$-separation displayed by $\Phi$. If $P_{1}$ and $P_{2}$ are both contained in either $R$ or $G$, then $(R, G)$ is displayed by $\Phi^{\prime}$. Thus we may assume, up to switching $R$ and $G$, that $P_{1} \subseteq G$ and $P_{2} \subseteq R$. We claim that the partition $\left(R \cup P_{1}, G-P_{1}\right)$ is a non-sequential $k$-separation that is $\mathcal{T}$-equivalent to $(R, G)$. The set $R \cup P_{1}$ is the union of the $k$-separating sets $P_{1} \cup P_{2}$ and $R$, and their intersection is $P_{2}$. Thus, by uncrossing $P_{1} \cup P_{2}$ and $R$, the set $R \cup P_{1}$ is $k$-separating. Moreover, $G-P_{1}$ contains some petal of $\Phi$ because $P_{1} \subseteq \operatorname{fcl}_{\mathcal{T}}\left(P_{2}\right) \subseteq \operatorname{fcl}_{\mathcal{T}}(R)$ by Lemma 3.3.3, and $(R, G)$ is a nonsequential $k$-separation. Thus $\left(R \cup P_{1}, G-P_{1}\right)$ is a $\mathcal{T}$-strong $k$-separation. Now from Lemma 3.3.3 it follows that $\mathrm{fcl}_{\mathcal{T}}\left(R \cup P_{1}\right)=\mathrm{fcl}_{\mathcal{T}}(R)$ and $\operatorname{fcl}_{\mathcal{T}}(G-$ $\left.P_{1}\right) \subseteq \mathrm{fcl}_{\mathcal{T}}(G)$, so $\left(R \cup P_{1}, G-P_{1}\right)$ is a non-sequential $k$-separation. Hence $\left(R \cup P_{1}, G-P_{1}\right) \mathcal{T}$-equivalent to $(R, G)$ by Lemma 3.3.11. Then $\Phi \preccurlyeq \Phi^{\prime}$, since $\left(R \cup P_{1}, G-P_{1}\right)$ is displayed by $\Phi^{\prime}$. Thus $\Phi$ and $\Phi^{\prime}$ are indeed equivalent $k$-flowers.

The remainder of this section is devoted to proving some lemmas about $k$-flowers in $\mathcal{T}$ that will be applied in Section 3.7 and Chapter 4 . Since the results are not used in the main goal of this Chapter, the proof of Theorem 3.6.1, the reader seeking a more direct path to this goal may proceed to Section 3.5 .

Lemma 3.4.5. Let $\mathcal{T}$ be a tangle of order $k$ in a connectivity system $(E, \lambda)$. If $\Phi=\left(P_{1}, P_{2}, \ldots, P_{n}\right)$ is a $k$-flower in $\mathcal{T}$ of order at least two, then $\mathrm{fcl}_{\mathcal{T}}\left(P_{i}\right) \neq E$ for all $i \in[n]$.

Proof. Seeking a contradiction, suppose that $\Phi$ is a $k$-flower of order at least two and that $\operatorname{fcl}_{\mathcal{T}}\left(P_{1}\right)=E$. Let $(R, G)$ be a $k$-separation displayed by $\Phi$. Up to switching $R$ and $G$, we may assume that $P_{1} \subseteq R$. Then $E=\operatorname{fcl}_{\mathcal{T}}\left(P_{1}\right) \subseteq \operatorname{fcl}_{\mathcal{T}}(R)$ by Lemma 3.3 .3 , so $\operatorname{fcl}_{\mathcal{T}}(R)=E$. Thus $(R, G)$ is a $\mathcal{T}$-sequential $k$-separation. Since $(R, G)$ was an arbitrary $k$-separation displayed by $\Phi$, it follows that every $k$-separation displayed by $\Phi$ is $\mathcal{T}$-sequential; 
a contradiction because $\Phi$ has order at least two.

Lemma 3.4.6. Let $\mathcal{T}$ be a tangle of order $k$ in a connectivity system $(E, \lambda)$, and let $\Phi=\left(P_{1}, P_{2}, \ldots, P_{n}\right)$ be a $k$-flower in $\mathcal{T}$ of order at least two, with no $\mathcal{T}$-loose petals. If $X \subseteq E-P_{1}$ is a non-empty $\mathcal{T}$-weak set such that $P_{1} \cup X$ is $k$-separating, then $P_{j}-X$ is $\mathcal{T}$-strong for all $j \in[2, n]$.

Proof. Let $X \subseteq E-P_{1}$ be a non-empty $\mathcal{T}$-weak set such that $P_{1} \cup X$ is $k$-separating.

3.4.6.1. There is some $j \in[2, n]$ such that $P_{j}-X$ is $\mathcal{T}$-strong.

Subproof. Seeking a contradiction, assume that $P_{j}-X$ is $\mathcal{T}$-weak for all $j \in$ $[2, n]$. Then, for $i \in[2, n-1]$, the set $P_{1} \cup P_{2} \cup \cdots \cup P_{i} \cup X$ is the union of the $k$ separating sets $P_{1} \cup P_{2} \cup \cdots \cup P_{i}$ and $P_{1} \cup X$. The set $P_{1} \cup\left(X \cap\left(P_{1} \cup P_{2} \cup \cdots \cup P_{i}\right)\right)$ is the intersection of $P_{1} \cup P_{2} \cup \cdots \cup P_{i}$ and $P_{1} \cup X$, and the partition

$$
\left(P_{1} \cup\left(X \cap\left(P_{1} \cup P_{2} \cup \cdots \cup P_{i}\right)\right),\left(\left(P_{2} \cup \cdots \cup P_{i}\right)-X\right) \cup P_{i+1} \cup \cdots \cup P_{n}\right)
$$

is $\mathcal{T}$-strong, so $\lambda\left(P_{1} \cup\left(X \cap\left(P_{1} \cup P_{2} \cup \cdots \cup P_{i}\right)\right)\right) \geq k$ by (T2). Thus, for all $i \in[2, n-1]$, the set $P_{1} \cup P_{2} \cup \cdots \cup P_{i} \cup X$ is $k$-separating by uncrossing $P_{1} \cup P_{2} \cup \cdots \cup P_{i}$ and $P_{1} \cup X$. Thus $\left(X, P_{2}-X, \ldots, P_{n}-X\right)$ is a partial $k$-sequence for $P_{1}$. By Lemma 3.3.5, we have $X \cup\left(P_{2}-X\right) \cup \cdots \cup\left(P_{n}-X\right) \subseteq$ $\mathrm{fcl}_{\mathcal{T}}\left(P_{1}\right)$, so $\mathrm{fcl}_{\mathcal{T}}\left(P_{1}\right)=E$; a contradiction of Lemma 3.4.5.

Assume that $P_{i}-X$ is $\mathcal{T}$-weak for some $i \in[2, n]$. Since there is some $j \in[2, n]$ such that $P_{j}-X$ is $\mathcal{T}$-strong by 3.4.6.1, we may assume, by reversing the order of the petals $P_{2}, \ldots, P_{n}$ of $\Phi$ if necessary, that $i \in[2, j-1]$. The set $P_{1} \cup \cdots \cup P_{i-1} \cup X$ is the union of the $k$-separating sets $P_{1} \cup X$ and $P_{1} \cup \cdots \cup P_{i-1}$, whose intersection is $P_{1} \cup\left(X \cap\left(P_{2} \cup \cdots \cup P_{i-1}\right)\right)$. Now, the partition

$$
\left(P_{1} \cup\left(X \cap\left(P_{2} \cup \cdots \cup P_{i-1}\right)\right),\left(\left(P_{2} \cup \cdots \cup P_{i-1}\right)-X\right) \cup P_{i} \cup \cdots \cup P_{n}\right)
$$

is $\mathcal{T}$-strong, so $\lambda\left(P_{1} \cup\left(X \cap\left(P_{2} \cup \cdots \cup P_{i-1}\right)\right)\right) \geq k$ by (T2). Thus $P_{1} \cup$ $\cdots \cup P_{i-1} \cup X$ is $k$-separating by uncrossing $P_{1} \cup X$ and $P_{1} \cup \cdots \cup P_{i-1}$. 
Another uncrossing argument with the $k$-separating sets $P_{1} \cup \cdots \cup P_{i-1} \cup X$ and $P_{i-1} \cup P_{i}$, whose union is a $\mathcal{T}$-strong set that avoids the $\mathcal{T}$-strong set $P_{j}-X$, shows that their intersection $P_{i-1} \cup\left(P_{i} \cap X\right)$ is $k$-separating. Then $\left(P_{i} \cap X, P_{i}-X\right)$ is a partial $k$-sequence for $P_{i-1}$, so $P_{i} \subseteq \operatorname{fcl}_{\mathcal{T}}\left(P_{i-1}\right)$ by Lemma 3.3.5; a contradiction because $\Phi$ has no $\mathcal{T}$-loose petals.

The next lemma relates equivalence of $k$-separations and equivalence of $k$-flowers in $\mathcal{T}$.

Lemma 3.4.7. Let $\Phi=\left(P_{1}, \ldots, P_{n}\right)$ be a $k$-flower in $\mathcal{T}$ of order at least two, with no $\mathcal{T}$-loose petals. Let $X \subseteq E-P_{1}$ be a non-empty $\mathcal{T}$-weak set such that $P_{1} \cup X$ is k-separating. Then $\Phi^{\prime}=\left(P_{1} \cup X, P_{2}-X, \ldots, P_{n}-X\right)$ is a $k$-flower in $\mathcal{T}$ that is equivalent to $\Phi$. Moreover, if we let $\left(P_{1}^{\prime}, \ldots, P_{n}^{\prime}\right)=$ $\left(P_{1} \cup X, P_{2}-X, \ldots, P_{n}-X\right)$, then $\operatorname{fcl}_{\mathcal{T}}\left(P_{i}^{\prime}\right)=\mathrm{fcl}_{\mathcal{T}}\left(P_{i}\right)$ for all $i \in[n]$.

Proof. We first show that $\Phi^{\prime}$ is a $k$-flower in $\mathcal{T}$. It follows from Lemma 3.4.6 that the partition $\Phi^{\prime}$ is $\mathcal{T}$-strong. It remains, then, to show that both any member of $\Phi^{\prime}$ and the union of any two consecutive members of $\Phi$ is $k$-separating. If $n=2$, then this follows from Lemma 3.3.9. Assume that $n=3$. Then, for each $i \in\{2,3\}$, it follows from uncrossing $E-P_{i}$ and $P_{1} \cup X$ that $\left(P_{1} \cup X\right) \cup\left(P_{j}-X\right)$ is $k$-separating for $j \in\{2,3\}-\{i\}$. Thus $\Phi^{\prime}$ is a $k$-flower. We may therefore assume that $n \geq 4$. The set $P_{1} \cup X \cup\left(P_{2}-X\right)$ is the union of the $k$-separating sets $P_{1} \cup P_{2}$ and $P_{1} \cup X$ whose intersection is $P_{1} \cup\left(P_{2} \cap X\right)$. The partition $\left(P_{1} \cup\left(P_{2} \cap X\right), E-\left(P_{1} \cup\left(P_{2} \cap X\right)\right)\right)$ is $\mathcal{T}$-strong, so $\lambda\left(P_{1} \cup\left(P_{2} \cap X\right)\right) \geq k$ by (T2). Thus $P_{1} \cup X \cup\left(P_{2}-X\right)$ is $k$-separating by uncrossing $P_{1} \cup P_{2}$ and $P_{1} \cup X$. Moreover, for each $i \in[2, n-1]$, the set $\left(P_{i}-X\right) \cup\left(P_{i+1}-X\right)$ is the intersection of the $k$-separating sets $E-\left(P_{1} \cup X\right)$ and $P_{i} \cup P_{i+1}$, whose union is a $\mathcal{T}$-strong set that avoids the $\mathcal{T}$-strong set $P_{1}$, so $\left(P_{i}-X\right) \cup\left(P_{i+1}-X\right)$ is $k$-separating by uncrossing. Thus $\Phi^{\prime}$ satisfies the hypotheses of Lemma 3.4.3, so $\Phi^{\prime}$ is indeed a $k$-flower in $\mathcal{T}$.

We now show that $\Phi^{\prime}$ is $\mathcal{T}$-equivalent to $\Phi$. Suppose that $(R, G)$ is a non-sequential $k$-separation displayed by $\Phi$. Then, up to switching $R$ and $G$, we may assume that $P_{1} \subseteq R$. Then $R \cup X$ is the union of the $k$-separating 
sets $P_{1} \cup X$ and $R$, whose intersection is $P_{1} \cup(R \cap X)$. Since the partition $\left(P_{1} \cup(R \cap X), G \cup\left(R-\left(P_{1} \cup X\right)\right)\right)$ is $\mathcal{T}$-strong, it follows from (T2) that $\lambda\left(P_{1} \cup(R \cap X)\right) \geq k$. Thus $R \cup X$ is $k$-separating by uncrossing $P_{1} \cup X$ and $R$. Thus $(R \cup X, G-X)$ is an equivalent $k$-separation to $(R, G)$ by Lemma 3.3.10. Moreover, the $k$-separation $(R \cup X, G-X)$ is displayed by $\Phi^{\prime}$. Thus $\Phi \preccurlyeq \Phi^{\prime}$. Now suppose that $(R, G)$ is a non-sequential $k$-separation that is displayed by $\Phi^{\prime}$. Then, up to switching $R$ and $G$, we may assume that $P_{1} \cup X \subseteq R$. Let $I=\left\{i \in[2, n] \mid P_{i}-X \subseteq G\right\}$. Then it is easy to check that $P_{I}$ is a $k$-separating set. It now follows that $G \cup\left(X \cap P_{I}\right)$ is $k$-separating by uncrossing $G$ and $P_{I}$, so $\left(G \cup\left(X \cap P_{I}\right), R-\left(X \cap P_{I}\right)\right)$ is a $k$-separation displayed by $\Phi$ that is equivalent to $(R, G)$ by Lemma 3.3.10. Thus $\Phi^{\prime} \preccurlyeq \Phi$. This establishes the first part.

For the second part, let $\left(P_{1}^{\prime}, \ldots, P_{n}^{\prime}\right)=\left(P_{1} \cup X, P_{2}-X, \ldots, P_{n}-X\right)$. Then, for all $i \in[2, n]$, we observe that $\left(P_{i} \cap X\right)$ is a partial $k$-sequence for $P_{i}-X$. Then it follows immediately from Corollary 3.3 .6 that $\operatorname{fcl}_{\mathcal{T}}\left(P_{i}^{\prime}\right)=\operatorname{fcl}_{\mathcal{T}}\left(P_{i}\right)$ for all $i \in[n]$.

The next result shows that certain concatenations of tight $k$-flowers in $\mathcal{T}$ have no $\mathcal{T}$-loose petals.

Lemma 3.4.8. Let $\Phi=\left(P_{1}, \ldots, P_{n}\right)$ be a tight $k$-flower in $\mathcal{T}$ of order at least three, and let $j \in[2, n-1]$. If $\left(P_{1} \cup \cdots \cup P_{j}, P_{j+1} \cup \cdots \cup P_{n}\right)$ is a non-sequential $k$-separation, then the concatenation $\Phi^{\prime}=\left(P_{1} \cup \cdots \cup P_{j}, P_{j+1}, \ldots, P_{n}\right)$ of $\Phi$ has no $\mathcal{T}$-loose petals.

Proof. Suppose that $\left(P_{1} \cup \cdots \cup P_{j}, P_{j+1} \cup \cdots \cup P_{n}\right)$ is a non-sequential $k$ separation, and let $J=[j]$. Let $\Phi^{\prime}=\left(P_{J}, P_{j+1}, \ldots, P_{n}\right)$ be the concatenation of $\Phi$. If $j=n-1$, then the lemma immediately holds, so we may assume that $j<n-1$. Seeking a contradiction, suppose that $\Phi^{\prime}$ has a $\mathcal{T}$-loose petal. Then $\Phi$ has no $\mathcal{T}$-loose petals by Lemma 3.4.4, so we may assume that $P_{j+1} \subseteq \operatorname{fcl}_{\mathcal{T}}\left(P_{J}\right)$. Let $\left(X_{i}\right)_{i=1}^{m}$ be a $k$-sequence for $P_{J}$. Then $P_{j+1} \subseteq$ $\bigcup_{i=1}^{m} X_{i}$. The partition $\left(P_{J} \cup P_{j+1} \cup X_{1}, E-\left(P_{J} \cup P_{j+1} \cup X_{1}\right)\right)$ is $\mathcal{T}$-strong because $E-\left(P_{J} \cup P_{j+1} \cup X_{1}\right)$ contains the $\mathcal{T}$-strong set $E-\mathrm{fcl}_{\mathcal{T}}\left(P_{J}\right)$. Thus 
$\lambda\left(P_{J} \cup P_{j+1} \cup X_{1}\right) \geq k$ by (T2). It now follows from uncrossing $P_{J} \cup X_{1}$ and $P_{j} \cup P_{j+1}$, whose union is $P_{J} \cup P_{j+1} \cup X_{1}$, that $P_{j} \cup\left(P_{j+1} \cap X_{1}\right)$ is $k$-separating. This process can clearly be repeated, so that $P_{j} \cup\left(\bigcup_{i=1}^{\ell}\left(P_{j+1} \cap X_{i}\right)\right)$ is $k$ separating for all $\ell \in[m]$. Then, up to removing any empty terms, the sequence $\left(P_{j+1} \cap X_{i}\right)_{i=1}^{m}$ is a partial $k$-sequence for $P_{j}$, so $P_{j+1} \subseteq \operatorname{fcl}_{\mathcal{T}}\left(P_{j}\right)$ by Lemma 3.3.5, a contradiction because $\Phi$ has no $\mathcal{T}$-loose petals.

Lemma 3.4.9. Let $\Phi=\left(P_{1}, \ldots, P_{n}\right)$ be a $k$-flower in $\mathcal{T}$ of order at least three, and let $(A, B)$ and $(C, D)$ be inequivalent non-sequential $k$-separations of $\lambda$ that are displayed by $\Phi$. If $\left(A^{\prime}, B^{\prime}\right)$ is $\mathcal{T}$-equivalent to $(A, B)$, and $A^{\prime} \subseteq$ $C$, then there is a $k$-separation $\left(A^{\prime \prime}, B^{\prime \prime}\right)$ that is $\mathcal{T}$-equivalent to $(A, B)$ and displayed by $\Phi$ with $A^{\prime \prime} \subseteq C$.

Proof. Suppose that $\left(A^{\prime}, B^{\prime}\right)$ is $\mathcal{T}$-equivalent to $(A, B)$ and that $A^{\prime} \subseteq C$. We may assume, up to labels, that $C=P_{1} \cup \cdots \cup P_{j}$ for some $j \in[n-1]$. Let $I \subseteq[n]$ be the set of indices such that $P_{I}=A$, and let $J=[j]$. Then $A^{\prime} \subseteq P_{J}$, so it follows from Lemma 3.3 .13 that $K=I \cap J$ is non-empty. Moreover, it is easily seen that $P_{K}$ is a $k$-separating set. Then, since $A \cap A^{\prime} \subseteq P_{K} \subseteq A$, it follows from Lemma 3.3 .14 that $\left(P_{K}, E-P_{K}\right)$ is a $k$-separation displayed by $\Phi$ that is $\mathcal{T}$-equivalent to $(A, B)$, and that $P_{K} \subseteq C$.

\subsection{Conformity}

Recall that the main goal of this chapter is to prove Theorem 3.6.1, which, loosely stated, is to show that if $\mathcal{T}$ is a tangle of order $k$ that satisfies a certain robustness condition, then every non-sequential $k$-separation conforms with a tight maximal flower in $\mathcal{T}$. In this section, we study how the non-sequential $k$-separations of $\lambda$ interact with $k$-flowers in $\mathcal{T}$, and we develop the necessary lemmas to prove Theorem 3.6.1.

To avoid cumbersome statements we assume throughout this section that $\mathcal{T}$ is a tangle of order $k$ in a connectivity system $(E, \lambda)$.

We begin with the following easy lemmas about certain subsets of petals of a $k$-flower $\Phi$ in $\mathcal{T}$. 
Lemma 3.5.1. Let $n \geq 2$, and let $I$ be a proper non-empty subset of $[n]$. Let $\Phi=\left(P_{1}, \ldots, P_{n}\right)$ be a $k$-flower in $\mathcal{T}$. If $X \subseteq P_{I}$ is a $\mathcal{T}$-strong set, then $\lambda(X) \geq k$.

Proof. Suppose that $X \subseteq P_{I}$ is a $\mathcal{T}$-strong set. Then there is some $i \in[n]-I$, so $P_{i} \subseteq E-X$. Thus $(X, E-X)$ is a $\mathcal{T}$-strong partition, so $\lambda(X) \geq k$ by (T2).

Lemma 3.5.2. Let $n \geq 2$, and let $I$ be a proper non-empty subset of $[n]$. Let $\Phi=\left(P_{1}, \ldots, P_{n}\right)$ be a $k$-flower in $\mathcal{T}$. If $X \subseteq P_{I}$ and $\lambda(X)<k$, then $X \in \mathcal{T}$.

Proof. Suppose that $X \subseteq P_{I}$ and $\lambda(X)<k$. Then $X$ or $E-X$ belongs to $\mathcal{T}$ by (T2), and $E-X$ is $\mathcal{T}$-strong, so $X \in \mathcal{T}$.

Let $n \geq 2$, and let $I \subseteq[n]$ be a proper non-empty subset. Let $\Phi=$ $\left(P_{1}, \ldots, P_{n}\right)$ be a $k$-flower in $\mathcal{T}$. A $k$-separation $(R, G)$ of $\lambda$ crosses a union of petals $P_{I}$ of $\Phi$ if both $P_{I} \cap R$ and $P_{I} \cap G$ are non-empty sets. We say that $P_{I}$ is strongly crossed by $(R, G)$ if both $P_{I} \cap R$ and $P_{I} \cap G$ are $\mathcal{T}$-strong sets, and that $P_{I}$ is weakly crossed by $(R, G)$ if both $P_{I} \cap R$ and $P_{I} \cap G$ are $\mathcal{T}$-weak sets.

A $\mathcal{T}$-strong $k$-separation $(R, G)$ is said to conform with a $k$-flower $\Phi$ in $\mathcal{T}$ if either $(R, G)$ is $\mathcal{T}$-equivalent to a $k$-separation that is displayed by $\Phi$ or $(R, G)$ is $\mathcal{T}$-equivalent to a $k$-separation $\left(R^{\prime}, G^{\prime}\right)$ with the property that $R^{\prime}$ or $G^{\prime}$ is contained in a petal of $\Phi$.

Let $\Phi$ be a $k$-flower in $\mathcal{T}$, and let $(R, G)$ be a non-sequential $k$-separation that does not conform with $\Phi$. Then it is easy to see that it crosses some petal of $\Phi$. We would like show that there is a $k$-flower that both refines $\Phi$ and displays a $k$-separation that is equivalent to $(R, G)$.

A $\mathcal{T}$-strong $k$-separation $(R, G)$ called $\Phi$-minimum if, among the $k$ separations that are $\mathcal{T}$-equivalent to $(R, G)$, it crosses a minimum number of petals of $\Phi$.

Lemma 3.5.3. Let $n \geq 2$, and let $\Phi=\left(P_{1}, \ldots, P_{n}\right)$ be a $k$-flower in $\mathcal{T}$. Let $I$ be a proper non-empty subset of $[n]$ such that $P_{I}$ is a k-separating, and 
let $(R, G)$ be a $\Phi$-minimum non-sequential $k$-separation that crosses $P_{I}$. If $\lambda\left(P_{I} \cap R\right) \geq k$, then $P_{I} \cap G$ is $\mathcal{T}$-strong.

Proof. Assume that $\lambda\left(P_{I} \cap R\right) \geq k$. Seeking a contradiction, suppose that $P_{I} \cap G$ is $\mathcal{T}$-weak. Then $P_{I} \cup R$ is $k$-separating by uncrossing $P_{I}$ and $R$, so $(R, G)$ is $\mathcal{T}$-equivalent to $\left(R \cup P_{I}, G-P_{I}\right)$ by Lemma 3.3.10. But $\left(R \cup P_{I}, G-\right.$ $\left.P_{I}\right)$ crosses fewer petals of $\Phi$ than $(R, G)$; a contradiction because $(R, G)$ is $\Phi$-minimum.

Lemma 3.5.4. Let $n \geq 2$, and let $\Phi=\left(P_{1}, \ldots, P_{n}\right)$ be a $k$-flower in $\mathcal{T}$. Let $I$ be a proper non-empty subset of $[n]$ such that $P_{I}$ is $k$-separating, and let $(R, G)$ be a $\Phi$-minimum non-sequential $k$-separation that crosses $P_{I}$. If $P_{I}$ is weakly crossed by $(R, G)$, then:

(i) $P_{I} \cap G$ and $P_{I} \cap R$ are both members of $\mathcal{T}$.

(ii) $P_{I}$ is a sequential $k$-separating set.

Proof. For (i), we show that $\lambda\left(P_{I} \cap R\right)<k$ and $\lambda\left(P_{I} \cap G\right)<k$. Seeking a contradiction, assume, up to switching $R$ and $G$, that $\lambda\left(P_{I} \cap R\right) \geq k$. Then $P_{I} \cap G$ is $\mathcal{T}$-strong by Lemma 3.5.3 a contradiction because $P_{I}$ is weakly crossed by $(R, G)$. Thus $\lambda\left(P_{I} \cap R\right)<k$ and $\lambda\left(P_{I} \cap G\right)<k$. It now follows from Lemma 3.5.2 that $P_{I} \cap G, P_{I} \cap R \in \mathcal{T}$.

We now prove (ii). The set $E-P_{I}$ is a $\mathcal{T}$-strong $k$-separating set because $I$ is a proper non-empty subset of $[n]$ such that $P_{I}$ is $k$-separating. Moreover, $P_{I} \cap G$ and $P_{I} \cap R$ are $\mathcal{T}$-weak sets, and $\lambda\left(\left(E-P_{I}\right) \cup\left(P_{I} \cap G\right)\right)=\lambda\left(P_{I} \cap R\right)<k$ by (i), so $\left(P_{I} \cap G, P_{I} \cap R\right)$ is a partial $k$-sequence for $E-P_{I}$. Thus $P_{I} \subseteq$ $\mathrm{fcl}_{\mathcal{T}}\left(E-P_{I}\right)$ by Lemma 3.3.5, and so $\mathrm{fcl}_{\mathcal{T}}\left(E-P_{I}\right)=E$.

The next lemma shows that a $k$-separating proper non-empty union of petals of $\Phi$ is either strongly or weakly crossed by a $\Phi$-minimum nonsequential $k$-separation $(R, G)$.

Lemma 3.5.5. Let $n \geq 2$, and let $\Phi=\left(P_{1}, \ldots, P_{n}\right)$ be a $k$-flower in $\mathcal{T}$. Let $I$ be a proper non-empty subset of $[n]$ such that $P_{I}$ is $k$-separating. If $(R, G)$ is 
a $\Phi$-minimum non-sequential $k$-separation that crosses $P_{I}$, then $P_{I}$ is either strongly or weakly crossed.

Proof. Assume that $(R, G)$ is a $\Phi$-minimum non-sequential $k$-separation that crosses $P_{I}$. If $\lambda\left(P_{I} \cap R\right)<k$ and $\lambda\left(P_{I} \cap G\right)<k$, then $(R, G)$ weakly crosses $P_{I}$ by Lemma 3.5.2. Thus we may assume, up to switching $R$ and $G$, that $\lambda\left(P_{I} \cap R\right) \geq k$. Then $P_{I} \cap G$ is $\mathcal{T}$-strong by Lemma 3.5.3. Thus $\lambda\left(P_{I} \cap G\right) \geq k$ by Lemma 3.5.1. Then $P_{I} \cap R$ is also $\mathcal{T}$-strong by Lemma 3.5.3. Therefore $(R, G)$ strongly crosses $P_{I}$.

The next lemma shows that if a non-sequential $k$-separation does not conform with a tight $k$-flower $\Phi$ in $\mathcal{T}$ of order two, then there is a $k$-flower in $\mathcal{T}$ that refines $\Phi$.

Lemma 3.5.6. Let $\Phi=\left(P_{1}, P_{2}\right)$ be a tight $k$-flower in $\mathcal{T}$. If $(R, G)$ is a non-sequential $k$-separation that does not conform with $\Phi$, then there is a $k$-flower $\Phi^{\prime}$ that refines $\Phi$ and displays a $k$-separation equivalent to $(R, G)$.

Proof. Suppose that $(R, G)$ is a non-sequential $k$-separation that does not conform with $\Phi$. We may assume, by possibly replacing $(R, G)$ by an equivalent $k$-separation, that $(R, G)$ is $\Phi$-minimum. Both $P_{1}$ and $P_{2}$ are crossed by $(R, G)$ because it does not conform with $\Phi$. We claim that $\Phi^{\prime}=\left(P_{1} \cap G, P_{1} \cap R, P_{2} \cap R, P_{2} \cap G\right)$ is a $k$-flower in $\mathcal{T}$. Since $\Phi$ is tight, the $k$-separation $\left(P_{1}, P_{2}\right)$ is non-sequential, so it follows from Lemma 3.5.4 (ii) that both $P_{1}$ and $P_{2}$ are strongly crossed by $(R, G)$. Thus $\Phi^{\prime}$ is a $\mathcal{T}$-strong partition. Furthermore, the union of any two consecutive petals of $\Phi^{\prime}$ is a member of $\left\{R, G, P_{1}, P_{2}\right\}$, and so $k$-separating. Thus $\Phi^{\prime}$ a $k$-flower in $\mathcal{T}$ by Lemma 3.4 .3 .

Let $n \geq 2$, and let $\Phi=\left(P_{1}, \ldots, P_{n}\right)$ be a $k$-flower in $\mathcal{T}$. Let $(R, G)$ be a $\Phi$ minimum non-sequential $k$-separation that does not conform with $\Phi$. Let $I$ be a proper non-empty subset of $[n]$ such that $P_{I}$ is $k$-separating. We say that $P_{I}$ is $(R, G)$-strong if either $P_{I}$ is not crossed by $(R, G)$ or $P_{I}$ is strongly crossed by $(R, G)$, and that $P_{I}$ is $(R, G)$-weak if $P_{I}$ is weakly crossed by $(R, G)$. By 
Lemma 3.5.5, $P_{I}$ is either $(R, G)$-weak or $(R, G)$-strong. Evidently, if a petal $P_{i}$ of $\Phi$ is $(R, G)$-strong, then $P_{i} \cap R$ or $P_{i} \cap G$ is $\mathcal{T}$-strong.

The next lemma shows that $(R, G)$-weak petals of $\Phi$ are the only obstacles to finding a $k$-flower that refines $\Phi$ and displays a $k$-separation that is equivalent to $(R, G)$.

Lemma 3.5.7. Let $n \geq 3$, and let $\Phi=\left(P_{1}, P_{2}, \ldots, P_{n}\right)$ be a $k$-flower in $\mathcal{T}$. Let $(R, G)$ be a $\Phi$-minimum non-sequential $k$-separation that does not conform with $\Phi$. If every petal of $\Phi$ is $(R, G)$-strong, then there is a $k$-flower that refines $\Phi$ and displays $(R, G)$.

Proof. Suppose that every petal of $\Phi$ is $(R, G)$-strong. Then, up to labels, we may assume that $(R, G)$ crosses $P_{1}$. Let $P_{3}^{\prime}=P_{3} \cup \cdots \cup P_{n}$.

3.5.7.1. Up to switching $R$ and $G$, both $P_{2} \cap R$ and $P_{3}^{\prime} \cap G$ are $\mathcal{T}$-strong.

Subproof. If $(R, G)$ crosses $P_{2}$, then both $P_{2} \cap R$ and $P_{2} \cap G$ are $\mathcal{T}$-strong. Up to switching $R$ and $G$, we may assume that $P_{3}^{\prime} \cap G$ is $\mathcal{T}$-strong, so both $P_{2} \cap R$ and $P_{3}^{\prime} \cap G$ are $\mathcal{T}$-strong. Thus we may assume that $(R, G)$ does not cross $P_{2}$. Then, up to switching $R$ and $G$, we can assume that $P_{2} \subseteq R$, so $P_{2} \cap R$ is $\mathcal{T}$-strong. Now if $G$ avoids $P_{3}^{\prime}$, then $G \subseteq P_{1}$; a contradiction because $(R, G)$ does not conform with $\Phi$. Thus $G$ meets $P_{3}^{\prime}$, so $P_{i} \cap G$ is $\mathcal{T}$-strong for some $i \in[3, n]$. Hence $P_{3}^{\prime} \cap G$ is $\mathcal{T}$-strong.

Assume that labels are chosen such that $P_{2} \cap R$ and $P_{3}^{\prime} \cap G$ are $\mathcal{T}$-strong.

3.5.7.2. $\Phi^{\prime}=\left(P_{1} \cap G, P_{1} \cap R, P_{2}, \ldots, P_{n}\right)$ is a $k$-flower in $\mathcal{T}$.

Subproof. The members of the partition $\Phi^{\prime}$ are $\mathcal{T}$-strong. Furthermore, $\left(P_{1} \cap\right.$ $G) \cup\left(P_{1} \cap R\right)$ is $k$-separating, and $P_{i} \cup P_{i+1}$ is $k$-separating for all $i \in[2, n-1]$. Thus, by Lemma 3.4 .3 , it suffices to show that $\left(P_{1} \cap R\right) \cup P_{2}$ is $k$-separating. Now $\lambda\left(P_{1} \cup P_{2} \cup R\right)=\lambda\left(P_{3}^{\prime} \cap G\right) \geq k$ by Lemma 3.5.1, so, by uncrossing $P_{1} \cup P_{2}$ and $R$, we see that $\left(P_{1} \cup P_{2}\right) \cap R$ is $k$-separating. Furthermore $\lambda\left(P_{2} \cap R\right) \geq k$ by Lemma 3.5.1, so $\left(P_{1} \cap R\right) \cup P_{2}$ is $k$-separating by uncrossing $P_{2}$ and $\left(P_{1} \cup P_{2}\right) \cap R$. 
It now follows from 3.5 .7 .2 and an induction on the number of petals of $\Phi$ crossed by $(R, G)$ that there is a $k$-flower $\Phi^{\prime}$ that refines $\Phi$ and displays $(R, G)$.

For tight $k$-flowers we only need two $(R, G)$-strong petals to guarantee that every petal is $(R, G)$-strong. To show this we first need the following technical lemma.

Lemma 3.5.8. Let $n \geq 3$, and let $\Phi=\left(P_{1}, \ldots, P_{n}\right)$ be a $k$-flower in $\mathcal{T}$. Let $(R, G)$ be a $\Phi$-minimum non-sequential $k$-separation that does not conform with $\Phi$. If $P_{1}$ is $(R, G)$-weak and there is a concatenation $\left(P_{1}, A, B\right)$ of $\Phi$ such that both $A$ and $B$ are $(R, G)$-strong, then $\Phi$ is equivalent to the $k$ flower $\Phi^{\prime}=\left(P_{1} \cup P_{2}, P_{3}, \ldots, P_{n}\right)$.

Proof. Suppose that $P_{1}$ is $(R, G)$-weak, and that $\left(P_{1}, A, B\right)$ is a concatenation of $\Phi$ such that both $A$ and $B$ are $(R, G)$-strong.

3.5.8.1. Up to switching $R$ and $G$, both $A \cap R$ and $B \cap G$ are $\mathcal{T}$-strong.

Subproof. Assume first that $(R, G)$ crosses $A$, so both $A \cap R$ and $A \cap G$ are $\mathcal{T}$-strong. Then, up to switching $R$ and $G$, we may assume that $B \cap G$ is $\mathcal{T}$-strong. Thus both $A \cap R$ and $B \cap G$ are $\mathcal{T}$-strong. Now assume that $(R, G)$ does not cross $A$. Then, up to switching $R$ and $G$, we may assume that $A \subseteq R$, so $A \cap R$ is $\mathcal{T}$-strong. If $B \subseteq R$, then $G \subseteq P_{1}$; a contradiction because $(R, G)$ does not conform with $\Phi$. Thus either $(R, G)$ crosses $B$ or $B \subseteq G$, so $B \cap G$ is $\mathcal{T}$-strong.

Assume that $R$ and $G$ are labelled such that both $A \cap R$ and $B \cap G$ are $\mathcal{T}$-strong. Then $\lambda(A \cap R) \geq k$ and $\lambda(B \cap G) \geq k$ by Lemma 3.5.1. Since $P_{1} \cup A \cup R$ avoids $B \cap G$, the set $\left(P_{1} \cup A\right) \cap R$ is $k$-separating by uncrossing $P_{1} \cup A$ and $R$. Another uncrossing argument with $\left(P_{1} \cup A\right) \cap R$ and $A$, whose intersection is $A \cap R$, shows that their union $A \cup\left(P_{1} \cap R\right)$ is $k$-separating. Finally, $P_{2} \cup\left(P_{1} \cap R\right)$ is the intersection of the $k$-separating sets $P_{1} \cup P_{2}$ and $A \cup\left(P_{1} \cap R\right)$, whose union is $A \cup P_{1}$, so $P_{2} \cup\left(P_{1} \cap R\right)$ is $k$-separating by uncrossing $P_{1} \cup P_{2}$ and $A \cup\left(P_{1} \cap R\right)$. Then $\left(P_{1} \cap R, P_{1} \cap G\right)$ is a partial 
$k$-sequence for $P_{2}$, so $P_{1} \subseteq \mathrm{fcl}_{\mathcal{T}}\left(P_{2}\right)$ by Lemma 3.3.5. Thus $\Phi$ is equivalent to the $k$-flower $\left(P_{1} \cup P_{2}, P_{3}, \ldots, P_{n}\right)$ by Lemma 3.4.4.

The next lemma shows that we only need to prove that a tight $k$-flower has two $(R, G)$-strong petals.

Lemma 3.5.9. Let $n \geq 3$, and let $\Phi=\left(P_{1}, \ldots, P_{n}\right)$ be a tight $k$-flower in $\mathcal{T}$. Let $(R, G)$ be a $\Phi$-minimal non-sequential $k$-separation that does not conform with $\Phi$. If $\Phi$ has two $(R, G)$-strong petals, then every petal of $\Phi$ is $(R, G)$-strong.

Proof. Assume that $\Phi$ has two $(R, G)$-strong petals. Seeking a contradiction, suppose that $\Phi$ has an $(R, G)$-weak petal. Then, up to labels, we may assume that $P_{1}$ is $(R, G)$-weak, and that $P_{2}$ is $(R, G)$-strong. Then $P_{j}$ is $(R, G)$ strong for some $j \notin[1,2]$, so $E-\left(P_{1} \cup P_{2}\right)$ is $(R, G)$-strong. Now $\left(P_{1}, P_{2}, E-\right.$ $\left.\left(P_{1} \cup P_{2}\right)\right)$ is a concatenation of $\Phi$ such that $P_{1}$ is $(R, G)$-weak, and both $P_{2}$ and $E-\left(P_{1} \cup P_{2}\right)$ are $(R, G)$-strong. Thus $\Phi$ is equivalent to the $k$-flower $\Phi^{\prime}=\left(P_{1} \cup P_{2}, P_{3}, \ldots, P_{n}\right)$ by Lemma 3.5.8, a contradiction because $\Phi$ is tight. Thus every petal of $\Phi$ is $(R, G)$-strong.

As a consequence of Lemma 3.5.9 and Lemma 3.5.4 we can refine tight $k$-flowers with three petals.

Lemma 3.5.10. Let $\Phi=\left(P_{1}, P_{2}, P_{3}\right)$ be a tight $k$-flower in $\mathcal{T}$. If $(R, G)$ is a non-sequential $k$-separation that does not conform with $\Phi$, then there is a $k$-flower that refines $\Phi$ and displays a $k$-separation equivalent to $(R, G)$.

Proof. Assume that $(R, G)$ is a $\Phi$-minimum non-sequential $k$-separation that does not conform with $\Phi$. As $\Phi$ is a tight $k$-flower in $\mathcal{T}$ of order three, it displays at least two inequivalent non-sequential $k$-separations. By Lemma 3.5.4 (ii) non-sequential $k$-separations displayed by $\Phi$ are strongly crossed by $(R, G)$, so we may assume that $P_{1}$ and $P_{2}$ are $(R, G)$-strong. Then all petals of $\Phi$ are $(R, G)$-strong by Lemma 3.5.9. It follows from Lemma 3.5.7 that there is a $k$-flower that refines $\Phi$ and displays a $k$-separation that is equivalent to $(R, G)$. 
In fact, we can do one better than Lemma 3.5.9.

Lemma 3.5.11. Let $n \geq 3$, and let $\Phi=\left(P_{1}, \ldots, P_{n}\right)$ be a tight $k$-flower in $\mathcal{T}$. Let $(R, G)$ be a $\Phi$-minimum non-sequential $k$-separation that does not conform with $\Phi$. If $\Phi$ has one $(R, G)$-strong petal, then every petal of $\Phi$ is $(R, G)$-strong.

Proof. If $\Phi$ has two $(R, G)$-strong petals, then the lemma immediately follows from Lemma 3.5.9. Assume towards a contradiction that $\Phi$ has exactly one $(R, G)$-strong petal. Then, up to labels, we can assume that $P_{2}$ is $(R, G)$ strong. Since $\Phi$ is a tight $k$-flower of order at least three, it displays some non-sequential $k$-separation $(X, Y)$ that is not equivalent to $\left(P_{2}, E-P_{2}\right)$. By Lemma 3.5.4 (ii) both $X$ and $Y$ are $(R, G)$-strong, so they must contain at least two petals of $\Phi$. Then we can assume that the petals of $\Phi$ and $(X, Y)$ are labelled such that $P_{2}$ is $(R, G)$-strong and $P_{1}, P_{2} \subseteq X$. Now $Y \subseteq E-\left(P_{1} \cup P_{2}\right)$, so $E-\left(P_{1} \cup P_{2}\right)$ is also $(R, G)$-strong. Thus $\left(P_{1}, P_{2}, E-\right.$ $\left.\left(P_{1} \cup P_{2}\right)\right)$ is a concatenation of $\Phi$ such that $P_{1}$ is $(R, G)$-weak, and both $P_{2}$ and $E-\left(P_{1} \cup P_{2}\right)$ are $(R, G)$-strong. Then it follows from Lemma 3.5.8 that $\Phi^{\prime}=\left(P_{1} \cup P_{2}, P_{3}, \ldots, P_{n}\right)$ is a $k$-flower equivalent to $\Phi$; a contradiction because $\Phi$ is tight. Thus $\Phi$ has two $(R, G)$-strong petals, so by Lemma 3.5.9 every petal of $\Phi$ is $(R, G)$-strong.

\subsection{Robustness}

Unfortunately, Lemma 3.5.11 is as much as we can say for arbitrary tangles in a connectivity system. Consider the 8 -element rank-4 matroid $R_{8}$ that is represented geometrically by a cube (see, for example, [22, pp. 508]).

The 4-point planes of $R_{8}$ are the six faces of the cube and the six diagonal planes. Let $E=[8]$ be the ground set of $R_{8}$, and let $r$ be the rank function of $R_{8}$. For each positive integer $\ell$, define a function $f_{\ell}$ on the subsets $X$ of $E$ by 


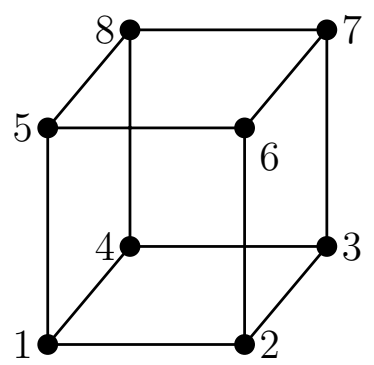

Figure 3.1: The matroid, $R_{8}$.

$$
f_{\ell}(X)= \begin{cases}0 & X=\emptyset . \\ r(X)+\ell & \text { otherwise. }\end{cases}
$$

It is straightforward to prove that $f_{\ell}$ is a polymatroid on $E=[8]$. Let $\lambda_{\ell}$ be the connectivity function of $f_{\ell}$, that is, $\lambda_{\ell}(X)=f_{\ell}(X)+f_{\ell}(E-X)-$ $f_{\ell}(E)+1$ for all $X \subseteq E$. Then $\mathcal{T}=\{\{i\} \mid i \in[8]\} \cup\{\emptyset\}$ is the unique tangle in $\left(E, \lambda_{\ell}\right)$ of order $\ell+3$. With notation as in Figure 3.1, the partition $\Phi=$ $(\{1,2\},\{3,4\},\{5,6\},\{7,8\})$ is a tight maximal $(\ell+3)$-flower in $\mathcal{T}$. However, the non-sequential $(\ell+3)$-separation $(\{1,3,5,7\},\{2,4,6,8\})$ does not conform with $\Phi$.

We can obtain analogous matroid examples by a standard construction where matroid elements are freely added to each polymatroid element.

Let $k$ be a positive integer, and let $\mathcal{T}$ be a collection of subsets of $E$ satisfying the axioms (T1), (T2), and (T4). Then $\mathcal{T}$ is a robust tangle in $(E, \lambda)$ of order $k$ if $\mathcal{T}$ satisfies:

(RT3) If $A_{1}, A_{2}, \ldots, A_{8} \in \mathcal{T}$, then $A_{1} \cup A_{2} \cup \cdots \cup A_{8} \neq E$.

Note that every robust tangle of order $k$ in $(E, \lambda)$ is certainly a tangle of order $k$ in $(E, \lambda)$.

A $k$-flower $\Phi$ in $\mathcal{T}$ is maximal if it is maximal in the quasi-order $\preccurlyeq$. We are now in position to achieve the main goal of this chapter. 
Theorem 3.6.1. Let $\mathcal{T}$ be a robust tangle of order $k$ in a connectivity system $(E, \lambda)$. If $\Phi$ is a tight maximal $k$-flower in $\mathcal{T}$, then every non-sequential $k$ separation of $\lambda$ conforms with $\Phi$.

Proof. Let $\Phi=\left(P_{1}, P_{2}, \ldots, P_{n}\right)$ be a tight maximal $k$-flower in $\mathcal{T}$. Assume that the theorem fails, and that $(R, G)$ is a $\Phi$-minimum non-sequential $k$ separation that does not conform with $\Phi$. Then clearly $n \geq 2$, and by Lemma 3.5.6 and Lemma 3.5.10, we may assume that $n \geq 4$. Assume towards a contradiction that every petal of $\Phi$ is $(R, G)$-weak. For each $i \in[n-3]$, let $A_{i}=P_{2} \cup P_{3} \cup \cdots \cup P_{n-i-1}$ and let $B_{i}=P_{n} \cup \cdots \cup P_{n-i+1}$, and consider the concatenation $\Phi_{i}=\left(P_{1}, A_{i}, P_{n-i}, B_{i}\right)$ of $\Phi$. The petals of $\Phi_{i}$ are either $(R, G)$-weak or $(R, G)$-strong by Lemma 3.5.5, and they cannot all be $(R, G)$ weak because $\mathcal{T}$ satisfies (RT3). For each $i \in[n-3]$, the petals $P_{1}$ and $P_{n-i}$ are $(R, G)$-weak, so $A_{i}$ or $B_{i}$ must be $(R, G)$-strong. Moreover, both $A_{1}$ and $B_{n-3}$ are $(R, G)$-strong because both $B_{1}=P_{n}$ and $A_{n-3}=P_{2}$ are $(R, G)$-weak. Thus there is a smallest index $j \geq 2$ such that $B_{j}$ is $(R, G)$ strong. Then $B_{j-1}$ is $(R, G)$-weak, so $A_{j-1}$ is $(R, G)$-strong by (RT3). Now $\left(P_{1}, A_{j-1}, B_{j}\right)$ is a concatenation of $\Phi$ such that both $A_{j-1}$ and $B_{j}$ are $(R, G)$ strong, so it follows from Lemma 3.5 .8 that $\Phi^{\prime}=\left(P_{1} \cup P_{2}, P_{3}, \ldots, P_{n}\right)$ is a $k$-flower equivalent to $\Phi$; a contradiction because $\Phi$ is tight. Thus $\Phi$ has an $(R, G)$-strong petal. It now follows from Lemma 3.5.11 that every petal of $\Phi$ is $(R, G)$-strong. Then, by Lemma 3.5.7, there is a $k$-flower that refines $\Phi$ and displays a $k$-separation that is equivalent to $(R, G)$; a contradiction of the maximality of $\Phi$.

We will later make use of the following conditions that are equivalent to (RT3) but are easier to verify. The proof is a simple extension of [9, Lemma $3.1]$.

Lemma 3.6.2. Let $(E, \lambda)$ be a connectivity system, and let $\mathcal{T}$ be a collection of subsets of $E$ satisfying the axioms (T1), (T2), and (T4). Then $\mathcal{T}$ is a robust tangle of order $k$ in $(E, \lambda)$ if and only if it satisfies the following conditions: 
(RT3a) If $A \subseteq B, B \in \mathcal{T}$, and $\lambda(A)<k$, then $A \in \mathcal{T}$.

(RT3b) If $\left(A_{1}, A_{2}, \ldots, A_{8}\right)$ is a partition of $E$, then $\mathcal{T}$ cannot contain all eight of $A_{1}, \ldots, A_{8}$.

Proof. If $\mathcal{T}$ is a robust tangle of order $k$ in $\lambda$, then it satisfies (RT3a) and (RT3b). Conversely, suppose that $\mathcal{T}$ satisfies (RT3a) and (RT3b). If $\mathcal{T}$ is not a robust tangle, then there are $A_{1}, A_{2}, \ldots, A_{8} \in \mathcal{T}$ such that $A_{1} \cup A_{2} \cup$ $\cdots \cup A_{8}=E$. Choose such $A_{1}, \ldots, A_{8}$ so that $\sum_{1 \leq i<j \leq 8}\left|A_{i} \cap A_{j}\right|$ is minimum. Then $\sum_{1 \leq i<j \leq 8}\left|A_{i} \cap A_{j}\right|>0$ by (RT3b), so we may assume, up to relabelling $A_{1}, A_{2}, \ldots, A_{8}$, that $\left|A_{1} \cap A_{2}\right|>0$. Then $\lambda\left(A_{1}-A_{2}\right)+\lambda\left(A_{2}-A_{1}\right) \leq \lambda\left(A_{1}\right)+$ $\lambda\left(A_{2}\right)$, so we may assume, up to relabelling $A_{1}$ and $A_{2}$, that $\lambda\left(A_{1}-A_{2}\right)<k$. Then $A_{1}-A_{2} \in \mathcal{T}$ by (RT3a). Let $B_{1}=A_{1}-A_{2}$ and let $B_{i}=A_{i}$ for $i \in[2,8]$. Then $B_{1}, \ldots, B_{8}$ cover $E$, and $\sum_{1 \leq i<j \leq 8}\left|B_{i} \cap B_{j}\right|<\sum_{1 \leq i<j \leq 8}\left|A_{i} \cap A_{j}\right|$, contradicting our choice of $A_{1}, \ldots, A_{8}$.

\subsection{Accumulating connectivity}

For a 3-connected matroid, if $\Phi=\left(P_{1}, \ldots, P_{n}\right)$ is a tight flower of order at least three, and $2 \leq j \leq n-1$, then $P_{1} \cup \cdots \cup P_{j}$ is a non-sequential 3separating set [23, Lemma 5.9 (ii)]. The aim of this section is to prove the existence of a bound on the number of petals in a sequential $k$-separating set displayed by a tight $k$-flower $\Phi$ in a tangle $\mathcal{T}$.

Let $\Phi=\left(P_{1}, P_{2}, \ldots, P_{n}\right)$ be a $k$-flower of order at least two with no $\mathcal{T}$ loose petals, and assume that $\bigcup_{i=1}^{\ell} P_{i}$ is sequential for some $\ell \in[n-1]$.

We first need the following corollary of Lemma 3.4.7.

Corollary 3.7.1. Let $\mathcal{T}$ be a tangle of order $k$ in a connectivity system $(E, \lambda)$. If $\Phi=\left(P_{1}, P_{2}, \ldots, P_{n}\right)$ is a $k$-flower in $\mathcal{T}$ of order at least two, with no $\mathcal{T}$-loose petals, and $\left(X_{i}\right)_{i=1}^{m}$ is a partial $k$-sequence for $P_{1}$, then $\Phi^{\prime}=$ $\left(P_{1}^{\prime}, P_{2}^{\prime}, \ldots, P_{n}^{\prime}\right)=\left(P_{1} \cup\left(\bigcup_{i=1}^{m} X_{i}\right), P_{2}-\left(\bigcup_{i=1}^{m} X_{i}\right), \ldots, P_{n}-\left(\bigcup_{i=1}^{m} X_{i}\right)\right)$ is a $k$-flower that is equivalent to $\Phi$. Moreover, $\operatorname{fcl}_{\mathcal{T}}\left(P_{i}\right)=\operatorname{fcl}_{\mathcal{T}}\left(P_{i}^{\prime}\right)$ for all $i \in[n]$. 
Proof. Assume that $\Phi=\left(P_{1}, P_{2}, \ldots, P_{n}\right)$ is a $k$-flower in $\mathcal{T}$ of order at least two, with no $\mathcal{T}$-loose petals. Assume that $\left(X_{i}\right)_{i=1}^{m}$ is a $k$-sequence for $P_{1}$. We proceed by induction on $m$. If $m=1$, then it follows from Lemma 3.4 .7 that the partition $\Phi_{1}=\left(P_{1}^{1}, P_{2}^{1}, \ldots, P_{n}^{1}\right)=\left(P_{1} \cup X_{1}, P_{2}-X_{1}, \ldots, P_{n}-X_{1}\right)$ is a $k$-flower in $\mathcal{T}$ that $\Phi_{1}$ is equivalent to $\Phi$, and that $\operatorname{fcl}_{\mathcal{T}}\left(P_{i}\right)=\operatorname{fcl}_{\mathcal{T}}\left(P_{i}^{1}\right)$ for all $i \in[n]$. Thus we may assume that $m \geq 2$, and that the result holds for $m-1$. Then $\left(X_{i}\right)_{i=1}^{m-1}$ is a partial $k$-sequence for $P_{1}$, so by induction there is a $k$-flower $\Phi_{m-1}=\left(P_{1}^{m-1}, P_{2}^{m-1}, \ldots, P_{n}^{m-1}\right)$, where $P_{1}^{m-1}=P_{1} \cup\left(\bigcup_{i=1}^{m-1} X_{i}\right)$ and $P_{j}^{m-1}=P_{j}-\left(\bigcup_{i=1}^{m-1} X_{i}\right)$ for $j \in[2, n]$, such that $\Phi_{m-1}$ is equivalent to $\Phi$, and $\operatorname{fcl}_{\mathcal{T}}\left(P_{i}\right)=\operatorname{fcl}_{\mathcal{T}}\left(P_{i}^{m-1}\right)$ for all $i \in[n]$. Thus $\Phi_{m-1}$ has order at least two, and no $\mathcal{T}$-loose petals. Now, by applying Lemma 3.4 .7 to the $k$-flower $\Phi_{m-1}$ and the $\mathcal{T}$-weak set $X_{m}$, it follows that $\Phi_{m}=\left(P_{1}^{m}, P_{2}^{m}, \ldots, P_{n}^{m}\right)$ is a $k$-flower in $\mathcal{T}$, where $P_{1}^{m}=P_{1} \cup\left(\bigcup_{i=1}^{m} X_{i}\right)$ and $P_{j}^{m}=P_{j}-\left(\bigcup_{i=1}^{m} X_{i}\right)$ for all $j \in[2, n]$, such that $\Phi_{m}$ is equivalent to $\Phi_{m-1}$, and hence is equivalent to $\Phi$, and $\operatorname{fcl}_{\mathcal{T}}\left(P_{i}^{m}\right)=\operatorname{fcl}_{\mathcal{T}}\left(P_{i}^{m-1}\right)=\operatorname{fcl}_{\mathcal{T}}\left(P_{i}\right)$ for all $i \in[n]$.

Lemma 3.7.2. Let $\mathcal{T}$ be a tangle of order $k$ in a connectivity system $(E, \lambda)$. If $\Phi=\left(P_{1}, P_{2}, \ldots, P_{n}\right)$ is a $k$-flower of order at least two, with no $\mathcal{T}$-loose petals, and $\bigcup_{i=1}^{\ell} P_{i}$ is sequential for some $\ell \in[n-1]$, then there is a $k$ flower $\Phi^{\prime}=\left(P_{1}^{\prime}, \ldots, P_{n}^{\prime}\right)$ that is equivalent to $\Phi$ with no $\mathcal{T}$-loose petals such that $\bigcup_{i=1}^{\ell} P_{i}^{\prime}$ sequential, and, for each $i \in[n-1], P_{i}^{\prime} \cap \mathrm{fcl}_{\mathcal{T}}\left(P_{j}^{\prime}\right)=\emptyset$ for all $j \in[i+1, n]$.

Proof. We describe a sequence of $k$-flowers in $\mathcal{T}$ obtained by applying Corollary 3.7.1. Assume that $\left(X_{i}\right)_{i=1}^{m}$ is a $k$-sequence for $P_{2}$, so $P_{2} \cup$ $\left(\bigcup_{i=1}^{m} X_{i}\right)=\mathrm{fcl}_{\mathcal{T}}\left(P_{2}\right)$ by Lemma 3.3.7. Then, by Corollary 3.7.1, the partition $\Phi^{\prime}=\left(P_{1}^{\prime}, P_{2}^{\prime}, \ldots, P_{n}^{\prime}\right)=\left(P_{1}-\mathrm{fcl}_{\mathcal{T}}\left(P_{2}\right), \operatorname{fcl}_{\mathcal{T}}\left(P_{2}\right), \ldots, P_{n}-\mathrm{fcl}_{\mathcal{T}}\left(P_{2}\right)\right)$ is a $k$-flower equivalent to $\Phi$. Moreover, we have $\operatorname{fcl}_{\mathcal{T}}\left(P_{i}\right)=\operatorname{fcl}_{\mathcal{T}}\left(P_{i}^{\prime}\right)$ for all $i \in[n]$. Now repeat this process with successive petals. In particular, after $n-1$ iterations, we obtain a $k$-flower $\Phi^{\prime \prime}=\left(P_{1}^{\prime \prime}, P_{2}^{\prime \prime}, \ldots, P_{n}^{\prime \prime}\right)$ with no $\mathcal{T}$-loose petals that is equivalent to $\Phi$, where $P_{1}^{\prime \prime}=P_{1}-\left(\bigcup_{i=2}^{n} \mathrm{fcl}_{\mathcal{T}}\left(P_{i}\right)\right)$, $P_{j}^{\prime \prime}=\mathrm{fcl}_{\mathcal{T}}\left(P_{j}\right)-\left(\bigcup_{i=j+1}^{n} \mathrm{fcl}_{\mathcal{T}}\left(P_{i}\right)\right)$ for $2 \leq j \leq n-1$, and $P_{n}^{\prime \prime}=\mathrm{fcl}_{\mathcal{T}}\left(P_{n}\right)$. Moreover, we have $\operatorname{fcl}_{\mathcal{T}}\left(P_{i}^{\prime \prime}\right)=\operatorname{fcl}_{\mathcal{T}}\left(P_{i}\right)$ for all $i \in[n]$. Thus, for each 
$i \in[n-1], P_{i}^{\prime \prime} \cap \operatorname{fcl}_{\mathcal{T}}\left(P_{j}^{\prime \prime}\right)=\emptyset$ for all $j \in[i+1, n]$. It follows from the fact that $\bigcup_{i=\ell+1}^{n} P_{i} \subseteq \bigcup_{i=\ell+1}^{n} P_{i}^{\prime \prime}$ and Lemma 3.3.3 that $\bigcup_{i=1}^{\ell} P_{i}^{\prime \prime}$ is a sequential $k$-separating set displayed by $\Phi^{\prime \prime}$ with $\ell$ petals.

Let $\Phi=\left(P_{1}, P_{2}, \ldots, P_{n}\right)$ be a $k$-flower of order at least two, with no $\mathcal{T}$ loose petals, and assume that $\bigcup_{i=1}^{\ell} P_{i}$ is sequential for some $\ell \in[n-1]$. Then it follows from Lemma 3.7 .2 that, by possibly replacing $\Phi$ by an equivalent $k$ flower $\Phi^{\prime}=\left(P_{1}^{\prime}, P_{2}^{\prime}, \ldots, P_{n}^{\prime}\right)$ that has no $\mathcal{T}$-loose petals and that also displays a sequential $k$-separating set $\bigcup_{i=1}^{\ell} P_{i}^{\prime}$, we may assume that, for each $i \in[n-1]$, $P_{i} \cap \operatorname{fcl}_{\mathcal{T}}\left(P_{j}\right)=\emptyset$ for all $j \in[i+1, n]$.

We now obtain a bound on the number of petals in a sequential $k$ separating set displayed by a $k$-flower in $\mathcal{T}$ of order at least two with no $\mathcal{T}$-loose petals.

Lemma 3.7.3. Let $\Phi=\left(P_{1}, P_{2}, \ldots, P_{n}\right)$ be a $k$-flower of order at least two with no $\mathcal{T}$-loose petals. If $\bigcup_{i=1}^{\ell} P_{i}$ is a sequential $k$-separation for some $\ell \in$ $[n-1]$, then $\ell \leq k$.

Proof. Suppose that $\bigcup_{i=1}^{\ell} P_{i}$ is a sequential $k$-separation for some $\ell \in[n-1]$. It follows from Lemma 3.7 .2 that, by possibly replacing $\Phi$ by an equivalent $k$ flower $\Phi^{\prime}=\left(P_{1}^{\prime}, P_{2}^{\prime}, \ldots, P_{n}^{\prime}\right)$ that has no $\mathcal{T}$-loose petals and that also displays a sequential $k$-separating set with $\ell$ petals, we may assume, for each $i \in$ $[n-1]$, that $P_{i} \cap \mathrm{fcl}_{\mathcal{T}}\left(P_{j}\right)=\emptyset$ for all $j \in[i+1, n]$. Since $\bigcup_{i=1}^{\ell} P_{i}$ is a sequential $k$-separation, there is a $k$-sequence $\left(X_{i}\right)_{i=1}^{m}$ for $P_{[\ell+1, n]}$. Let $X=X_{1}$.

3.7.3.1. $X$ meets $P_{1}$.

Subproof. Seeking a contradiction, suppose that $X$ avoids $P_{1}$. Let $i \in[2, \ell]$ be the smallest index such that $X \cap P_{i}$ is non-empty, so $X \subseteq P_{[i, \ell]}$. Then $P_{[i+1, n]} \cup X$ is the union of the $k$-separating sets $P_{[\ell+1, n]} \cup X$ and $P_{[i+1, n]}$. The intersection of $P_{[\ell+1, n]} \cup X$ and $P_{[i+1, n]}$ is $P_{[\ell+1, n]} \cup\left(X-P_{i}\right)$, and the partition $\left(P_{[\ell+1, n]} \cup\left(X-P_{i}\right), E-\left(P_{[\ell+1, n]} \cup\left(X-P_{i}\right)\right)\right)$ is $\mathcal{T}$-strong, so $\lambda\left(P_{[\ell+1, n]} \cup\right.$ $\left.\left(X-P_{i}\right)\right) \geq k$ by (T2). Thus, by uncrossing $P_{[\ell+1, n]} \cup X$ and $P_{[i+1, n]}$, the set $P_{[i+1, n]} \cup X$ is $k$-separating. Now, $P_{i+1} \cup\left(X \cap P_{i}\right)$ is the intersection 
of the $k$-separating sets $P_{i} \cup P_{i+1}$ and $P_{[i+1, n]} \cup X$, whose union is $P_{[i, n]}$, so $P_{i+1} \cup\left(X \cap P_{i}\right)$ is $k$-separating by uncrossing $P_{i} \cup P_{i+1}$ and $P_{[i+1, n]} \cup X$. Then $\left(X \cap P_{i}\right)$ is a partial $k$-sequence for $P_{i+1}$, so $X \cap P_{i} \subseteq \operatorname{fcl}_{\mathcal{T}}\left(P_{i+1}\right)$ by Lemma 3.3.5; a contradiction because $P_{i} \cap \mathrm{fcl}_{\mathcal{T}}\left(P_{i+1}\right)=\emptyset$.

3.7.3.2. $X$ meets $P_{i}$ for all $i \in[\ell]$.

Subproof. Seeking a contradiction, suppose that $X$ avoids some petal of $P_{i}$ for some $i \in[\ell]$. By 3.7.3.1 the set $P_{1} \cap X$ is non-empty, so $i \in[2, \ell]$. Now, $P_{n} \cup\left(P_{1} \cap X\right)$ is the intersection of the $k$-separating sets $P_{[\ell+1, n]} \cup X$ and $P_{n} \cup P_{1}$, whose union is $P_{1} \cup P_{[\ell+1, n]} \cup X$. Since $E-\left(P_{1} \cup P_{[\ell+1, n]} \cup X\right)$ contains $P_{i}$, the partition $\left(P_{1} \cup P_{[\ell+1, n]} \cup X, E-\left(P_{1} \cup P_{[\ell+1, n]} \cup X\right)\right)$ is clearly $\mathcal{T}$-strong. Then $\lambda\left(P_{1} \cup P_{[\ell+1, n]} \cup X\right) \geq k$ by (T2), so $P_{n} \cup\left(P_{1} \cap X\right)$ is $k$ separating by uncrossing $P_{[\ell+1, n]} \cup X$ and $P_{n} \cup P_{1}$. Thus $\left(P_{1} \cap X\right)$ is a partial $k$-sequence for $P_{n}$, so $P_{1} \cap X \subseteq \mathrm{fcl}_{\mathcal{T}}\left(P_{n}\right)$ by Lemma 3.3.5, a contradiction because $P_{1} \cap \operatorname{fcl}_{\mathcal{T}}\left(P_{n}\right)=\emptyset$.

By 3.7.3.2, the set $X \cap P_{i}$ is non-empty for all $i \in[\ell]$.

3.7.3.3. For each $i \in[\ell-1]$,

(i) $P_{[i]}-X \in \mathcal{T}$; and

(ii) $\lambda\left(P_{[i]}-X\right)>0$.

Subproof. For (i), by Lemma 3.5.2, it suffices to show that $\lambda\left(P_{[i]}-X\right)<k$. Seeking a contradiction, suppose that $\lambda\left(P_{[i]}-X\right) \geq k$ for some $i \in[\ell-1]$. Now, the set $P_{\ell+1} \cup\left(P_{[i+1, \ell]} \cap X\right)$ is the intersection of the $k$-separating sets $P_{[\ell+1, n]} \cup X$ and $P_{[i+1, \ell+1]}$. The union of $P_{[\ell+1, n]} \cup X$ and $P_{[i+1, \ell+1]}$ is $P_{[i+1, n]} \cup X$, whose complement is $P_{[i]}-X$. Thus $P_{\ell+1} \cup\left(P_{[i+1, \ell]} \cap X\right)$ is $k$-separating by uncrossing $P_{[\ell+1, n]} \cup X$ and $P_{[i+1, \ell+1]}$. Then $\left(P_{[i+1, \ell]} \cap X\right)$ is a partial $k$-sequence for $P_{\ell+1}$, so $P_{[i+1, \ell]} \cap X \subseteq \operatorname{fcl}_{\mathcal{T}}\left(P_{\ell+1}\right)$ by Lemma 3.3.5; a contradiction because $P_{[i+1, \ell]} \cap \mathrm{fcl}_{\mathcal{T}}\left(P_{\ell+1}\right)$ is empty.

For (ii), suppose that $\lambda\left(P_{[i]}-X\right) \leq 0$ for some $i \in[\ell-1]$. Then $P_{[i]}-X \in \mathcal{T}$ by (i). Moreover, the set $P_{i+1} \cup\left(P_{[i]}-X\right)$ is $k$-separating since, by the 
submodularity of $\lambda$, we have $\lambda\left(P_{i+1} \cup\left(P_{[i]}-X\right)\right) \leq \lambda\left(P_{i+1}\right)+\lambda\left(P_{[i]}-X\right) \leq k$. Thus, $\left(P_{[i]}-X\right)$ is a partial $k$-sequence for $P_{i+1}$, so $P_{[i]}-X \subseteq \operatorname{fcl}_{\mathcal{T}}\left(P_{i+1}\right)$ by Lemma 3.3.5, a contradiction because $P_{[i]} \cap \mathrm{fcl}_{\mathcal{T}}\left(P_{i+1}\right)=\emptyset$.

3.7.3.4. If $i \in[\ell-1]$, then $\lambda\left(P_{[i]}-X\right) \geq i$.

Subproof. We proceed by induction on $i$. If $i=1$, then the result follows from 3.7.3.3 (ii). Let $i \geq 2$, and assume for induction that $\lambda\left(P_{[i-1]}-X\right) \geq i-1$. Suppose that $\lambda\left(P_{[i]}-X\right) \leq i-1$. Then $\lambda\left(P_{[i-1]}-X\right) \geq \lambda\left(P_{[i]}-X\right)$. Thus, by submodularity, it follows that

$$
\lambda\left(P_{[i-1]} \cup\left(P_{i}-X\right)\right) \leq \lambda\left(P_{[i-1]}\right)+\lambda\left(P_{[i]}-X\right)-\lambda\left(P_{[i-1]}-X\right) \leq k .
$$

Now, the set $P_{i}-X$ is $\mathcal{T}$-weak by 3.7.3.3 (ii). Moreover, the set $P_{i-1} \cup\left(P_{i}-X\right)$ is the intersection of the $k$-separating sets $P_{i-1} \cup P_{i}$ and $P_{[i-1]} \cup\left(P_{i}-X\right)$, whose union is $P_{[i]}$, so $P_{i-1} \cup\left(P_{i}-X\right)$ is $k$-separating by uncrossing $P_{i-1} \cup P_{i}$ and $P_{[i-1]} \cup\left(P_{i}-X\right)$. Thus $\left(P_{i}-X, P_{i} \cap X\right)$ is a partial $k$-sequence for $P_{i-1}$, so $P_{i} \subseteq \operatorname{fcl}_{\mathcal{T}}\left(P_{i-1}\right)$ by Lemma 3.3.5, a contradiction because $\Phi$ has no $\mathcal{T}$-loose petals. Thus $\lambda\left(P_{[i]}-X\right) \geq i$, as required.

Thus, by 3.7.3.4, we have $\lambda\left(P_{[\ell-1]}-X\right) \geq \ell-1$. But we also have $\lambda\left(P_{[\ell-1]}-X\right)<k$ by 3.7.3.3(i) and (T1). Thus $\ell \leq k$, as required. 


\section{Chapter 4}

\section{Tree decomposition}

In this chapter, we show that, for every robust tangle of order $k$ in a connectivity system $(E, \lambda)$, there is an associated tree that displays, up to equivalence, all of the non-sequential $k$-separations of $\lambda$.

\subsection{Partial $k$-trees}

The tree used to obtain the tree decomposition of 3-connected matroids in [23] was a $\pi$-labelled tree called a maximal partial 3 -tree. We will use an analogous $\pi$-labelled tree to obtain the tree decomposition in Theorem 4.2.1. The exposition given here will therefore closely follow that of [23].

Let $\mathcal{T}$ be a tangle of order $k$ in a connectivity system $(E, \lambda)$, and let $\pi$ be a partition of $E$. Recall that we allow members of $\pi$ to be empty. Let $T$ be a tree such that every member of $\pi$ labels a vertex of $T$. Some vertices may be unlabelled and no vertex is multiply labelled. We say that $T$ is a $\pi$-labelled tree for $\mathcal{T}$. The vertices of $T$ labelled by the members of $\pi$ are called bag vertices, and the members of $\pi$ are called bags. A terminal bag is a bag that labels a leaf of $T$.

Let $T$ be a $\pi$-labelled tree for $\mathcal{T}$. We now define some partitions of $E$ that are induced by certain subgraphs of $T$. Let $T^{\prime}$ be a subtree of $T$. The union of those bags that label vertices of $T^{\prime}$ is the subset of $E$ displayed by 
$T^{\prime}$. Let $e$ be an edge of $T$. The partition of $E$ displayed by $e$ is the partition displayed by the connected components of $T \backslash e$. Let $v$ be a vertex of $T$ that is not a bag vertex. Then the partition of $E$ displayed by $v$ is the partition displayed by the connected components of $T-v$. The edges incident with $v$ are in natural one-to-one correspondence with the connected components of $T-v$, and hence with the members of the partition of $E$ displayed by $v$. In what follows, if a cyclic ordering is imposed on the edges incident with $v$, then we cyclically order the members of the partition of $E$ displayed by $v$ in the corresponding order.

Let $v$ be a vertex of $T$ that is not a bag vertex, and let $\left(e_{1}, \ldots, e_{n}\right)$ be a cyclic ordering of the edges incident with $v$. Then $v$ is a $k$-flower vertex if the partition $\left(P_{1}, \ldots, P_{n}\right)$ of $E$ displayed by $v$, in the cyclic order corresponding to $\left(e_{1}, \ldots, e_{n}\right)$, is a $k$-flower in $\mathcal{T}$. The $k$-separations displayed by the $k$-flower corresponding to a $k$-flower vertex are called the $k$-separations displayed by $v$. A $k$-separation is displayed by $T$ if it is displayed by some edge or some $k$-flower vertex of $T$. A $k$-separation $(X, Y)$ conforms with $T$ if either $(X, Y)$ is $\mathcal{T}$-equivalent to a $k$-separation displayed by $T$, or $(X, Y)$ is $\mathcal{T}$-equivalent to a $k$-separation $\left(X^{\prime}, Y^{\prime}\right)$ with the property that $X^{\prime}$ or $Y^{\prime}$ is contained in a bag of $T$.

Let $\mathcal{T}$ be a tangle of order $k$ in a connectivity system $(E, \lambda)$. A partial $k$-tree for $\mathcal{T}$ is a $\pi$-labelled tree for $\mathcal{T}$, where $\pi$ is a partition of $E$ such that the following properties hold:

(P1) For each edge $e$ of $T$, the partition $(X, Y)$ of $E$ displayed by $e$ is a $\mathcal{T}$-strong $k$-separation, and, if $e$ is incident with two bag vertices, then $(X, Y)$ is a non-sequential $k$-separation.

(P2) Each non-bag vertex $v$ of $T$ is labelled either $D$ or $A$. Moreover, if $v$ is labelled by $D$, then there is a cyclic ordering on the edges incident with $v$.

(P3) If a vertex $v$ if labelled by $A$, then the partition of $E$ displayed by $v$ is a $k$-anemone of order at least three with no $\mathcal{T}$-loose petals. 
(P4) If a vertex $v$ is labelled by $D$, then the partition of $E$ displayed by $v$, in the cyclic order induced by the cyclic ordering on the edges incident with $v$, is a $k$-daisy of order at least three with no $\mathcal{T}$-loose petals.

(P5) Every non-sequential $k$-separation conforms with $T$.

Note that we may suppress the labels $D$ or $A$ on non-bag vertices when they are clear from the context.

We now define a relation $\preccurlyeq$ on the set of partial $k$-trees for $\mathcal{T}$. Let $T$ and $T^{\prime}$ be partial $k$-trees for $\mathcal{T}$. If, for each non-sequential $k$-separation displayed by $T$, there is some $\mathcal{T}$-equivalent $k$-separation displayed by $T^{\prime}$, then $T \preccurlyeq T^{\prime}$. If $T \preccurlyeq T^{\prime}$ and $T^{\prime} \preccurlyeq T$, then $T$ is $\mathcal{T}$-equivalent to $T^{\prime}$. It is straightforward to prove that $\preccurlyeq$ is a quasi-order on the set of partial $k$-trees for $\mathcal{T}$. As with the other notions of equivalence we have developed, when the tangle $\mathcal{T}$ is clear from the context, we shall abbreviate "T -equivalent" to "equivalent". A partial $k$-tree is maximal if it is maximal in the quasi-order $\preccurlyeq$. A partial $k$-tree for $\mathcal{T}$ is trivial if it does not display any non-sequential $k$-separations.

Let $\Phi=\left(P_{1}, \ldots, P_{n}\right)$ be a $k$-flower in $\mathcal{T}$. Then there is a $\Phi$-labelled tree for $\mathcal{T}$ that we can associate with $\Phi$. If $n=1$, then $T$ consists of a single bag-vertex labelled by the bag $P_{1}$. If $n=2$, then $T$ consists of two adjacent bag vertices labelled by $P_{1}$ and $P_{2}$ respectively. Assume that $n \geq 3$. Then we let $T$ be the graph with vertex set $\left\{v, v_{1}, \ldots, v_{n}\right\}$, where $v$ is adjacent to each $v_{i}$ and each $v_{i}$ is labelled by the bag $P_{i}$. Finally, if $\Phi$ is a $k$-daisy, then the edges incident with the non-bag vertex $v$ are given the cyclic ordering $\left(v v_{1}, \ldots, v v_{n}\right)$. If $\Phi$ is a tight $k$-flower in $\mathcal{T}$, then it is easily seen that the associated $\Phi$-labelled tree for $\mathcal{T}$ satisfies the first four partial $k$-tree axioms. Moreover, we have the following immediate consequence of Theorem 3.6.1.

Corollary 4.1.1. Let $\mathcal{T}$ be a robust tangle of order $k$ in a connectivity system $(E, \lambda)$. If $\Phi$ is a tight maximal $k$-flower in $\mathcal{T}$, then the $\Phi$-labelled tree associated with $\Phi$ is a partial $k$-tree for $\mathcal{T}$.

The next result is used in the proof of Theorem 4.2.1. 
Lemma 4.1.2. Let $\mathcal{T}$ be a tangle of order $k$ in a connectivity system $(E, \lambda)$. If $(R, G)$ is a non-sequential $k$-separation of $\lambda$, then there is a tight maximal $k$-flower in $\mathcal{T}$ that displays a $k$-separation $\mathcal{T}$-equivalent to $(R, G)$.

Proof. Assume that $(R, G)$ is a non-sequential $k$-separation of $\lambda$. Then $\Phi=$ $(R, G)$ is a tight $k$-flower in $\mathcal{T}$, and evidently $\Phi$ displays $(R, G)$. Let $\Phi^{\prime}$ be a maximal $k$-flower in $\mathcal{T}$ such that $\Phi^{\prime} \succcurlyeq \Phi$, and let $\Phi^{\prime \prime}$ be a tight $k$-flower in $\mathcal{T}$ that is equivalent to $\Phi^{\prime}$. Then $\Phi^{\prime \prime}$ is a tight maximal $k$-flower in $\mathcal{T}$, and $\Phi^{\prime \prime} \succcurlyeq \Phi$, so $\Phi^{\prime \prime}$ displays a $k$-separation $\left(R^{\prime}, G^{\prime}\right)$ that is $\mathcal{T}$-equivalent to $(R, G)$.

The remainder of this section is devoted to developing the preliminary lemmas needed to prove the following lemma, which is the main component in the proof of Theorem 4.2.1.

Lemma 4.1.3. Let $\mathcal{T}$ a robust tangle of order $k$ in a connectivity system $(E, \lambda)$, and let $T$ be a non-trivial partial $k$-tree for $\mathcal{T}$. If there is a nonsequential $k$-separation $(R, G)$ that is not $\mathcal{T}$-equivalent to any $k$-separation displayed by $T$, then there is a partial $k$-tree $T^{\prime}$ such that $T^{\prime} \succcurlyeq T$ and $T^{\prime}$ displays some non-sequential $k$-separation that is not displayed by $T$.

Let $T$ be a partial $k$-tree for $\mathcal{T}$. If $B$ is a terminal bag of $T$ such that the partition $(B, E-B)$ is a non-sequential $k$-separation, then $B$ is called a non-sequential terminal bag of $T$. The main step towards a proof of Lemma 4.1 .3 is to show that if $T$ has a non-sequential terminal bag $B$, and $(C, E-C)$ is a non-sequential $k$-separation such that $\mathrm{fcl}_{\mathcal{T}}(B)=\mathrm{fcl}_{\mathcal{T}}(C)$, then there is some partial $k$-tree $T^{\prime}$ equivalent to $T$ such that $C$ is a terminal bag of $T^{\prime}$.

Recall that two separations $(A, B)$ and $(C, D)$ of $\mathcal{T}$ cross if the intersections $A \cap C, A \cap D, B \cap C$, and $B \cap D$ are all non-empty. A set $\mathcal{S}$ of separations of $\mathcal{T}$ is laminar if no two separations in $\mathcal{S}$ cross. We have the following straightforward lemma. We omit the details.

Lemma 4.1.4. If $T$ is a partial $k$-tree for $\mathcal{T}$, then the set of $k$-separations displayed by edges of $T$ is laminar. 
Lemma 4.1.5. Let $T$ be a partial $k$-tree for $\mathcal{T}$, and let $B$ be a terminal bag of $T$. If $\Phi=\left(P_{1}, P_{2}, \ldots, P_{n}\right)$ is a $k$-flower corresponding to a $k$-flower vertex $v$ of $T$, then, up to labels, $B \subseteq P_{1}$.

Proof. Suppose that $u$ is the bag vertex of $T$ labelled by $B$. Then $u$ is in some component of $T-v$, and hence $B$ is contained in some member of the partition of $E$ displayed by $v$. The members of the partition of $E$ displayed by $v$ are precisely the petals of $\Phi$, so, up to labels, we may assume that $B \subseteq P_{1}$.

Let $T$ be a partial $k$-tree for $\mathcal{T}$, and let $B$ be a non-sequential terminal bag of $T$. Suppose that $X \subseteq E-B$ is a $\mathcal{T}$-weak set such that $B \cup X$ is $k$-separating. We next show that there is a partial $k$-tree $T^{\prime}$ equivalent to $T$ with terminal bag $B \cup X$.

Lemma 4.1.6. Let $T$ be a partial $k$-tree for $\mathcal{T}$, and let $B$ be a non-sequential terminal bag of $T$ labelling a leaf $w$ of $T$. If $X \subseteq E-B$ is a non-empty $\mathcal{T}$ weak set such that $B \cup X$ is $k$-separating, then there is a partial $k$-tree $T^{\prime}$ equivalent to $T$ such that $B \cup X$ is a non-sequential terminal bag of $T^{\prime}$.

Proof. Suppose that $X \subseteq E-B$ is a non-empty $\mathcal{T}$-weak set such that $B \cup X$ is $k$-separating. If $\Phi=\left(P_{1}, \ldots, P_{n}\right)$ is a $k$-flower in $\mathcal{T}$ corresponding to a $k$-flower vertex of $T$, then we may assume that the petals of $\Phi$ are labelled such that $B \subseteq P_{1}$ by Lemma 4.1.5. Let $T^{\prime}$ be the $\pi$-labelled tree obtained by relabelling $T$ such that:

(i) the leaf $w$ of $T^{\prime}$ is labelled by the bag $B \cup X$, and, if $u \neq w$ is a bag vertex of $T$ labelled by the bag $B^{\prime}$, then $u$ is a bag vertex of $T^{\prime}$ labelled by the bag $B^{\prime}-X$; and

(ii) if $v$ is a non-bag vertex of $T$ labelled by $X \in\{D, A\}$, then $v$ is a non-bag vertex of $T^{\prime}$ labelled by $X$. Moreover, if a cyclic ordering is imposed on the edges of $T$ that are incident with $v$, then the cyclic ordering is imposed on the edges of $T^{\prime}$ that are incident with $v$. 
It is clear that $T$ and $T^{\prime}$ have the same bag vertices and non-bag vertices. We also see that $B \cup X$ is a non-sequential terminal bag of $T^{\prime}$ by (i) and Lemma 3.3.10. It remains, then, to show that $T^{\prime}$ is a partial $k$-tree for $\mathcal{T}$ that is equivalent to $T$. It follows immediately from (ii) that $T^{\prime}$ satisfies (P2). In the following three sublemmas we show that $T^{\prime}$ satisfies the remaining partial $k$-tree axioms.

4.1.6.1. If $v$ is a non-bag vertex of $T^{\prime}$, and $\Phi$ is the $k$-flower corresponding to the $k$-flower vertex $v$ of $T$, then the partition of $E$ displayed by the components of $T^{\prime}-v$ is a $k$-flower $\Phi^{\prime}$ that is $\mathcal{T}$-equivalent to $\Phi$ and has no $\mathcal{T}$-loose petals.

Subproof. Assume that the vertex $v$ of $T^{\prime}$ is a non-bag vertex, and that $\Phi=\left(P_{1}, P_{2}, \ldots, P_{n}\right)$ is the $k$-flower corresponding to the $k$-flower vertex $v$ of $T$. Then the partition of $E$ displayed by $T^{\prime}-v$, with the same ordering of the components as $T-v$, is $\Phi^{\prime}=\left(P_{1} \cup X, P_{2}-X, \ldots, P_{n}-X\right)$. The set $P_{1} \cup X$ is the union of the $k$-separating sets $P_{1}$ and $B \cup X$, whose intersection is $B \cup\left(P_{1} \cap X\right)$. Since both $B \cup\left(P_{1} \cap X\right)$ and $E-\left(B \cup\left(P_{1} \cap X\right)\right)$ are $\mathcal{T}$-strong, it follows from (T2) that $\lambda\left(B \cup\left(P_{1} \cap X\right)\right) \geq k$. Thus, by uncrossing, the set $P_{1} \cup X$ is $k$-separating. It now follows from Lemma 3.4.7 that $\Phi^{\prime}$ is a $k$-flower that is equivalent to $\Phi$, and that $\Phi^{\prime}$ has no $\mathcal{T}$-loose petals.

4.1.6.2. If $e$ is an edge of $T^{\prime}$, then the partition of $E$ displayed by the components of $T^{\prime} \backslash e$ is a $\mathcal{T}$-strong $k$-separation. Moreover, if $e$ is incident with two bag vertices of $T^{\prime}$, then the partition of $E$ displayed by the components of $T^{\prime} \backslash e$ is a non-sequential $k$-separation that is $\mathcal{T}$-equivalent to the $k$-separation displayed by the components of $T \backslash e$.

Subproof. Let $e$ be an edge of $T^{\prime}$. If $e$ is incident with a $k$-flower vertex of $T^{\prime}$, then the partition of $E$ displayed by the components of $T^{\prime} \backslash e$ is a $\mathcal{T}$-strong $k$-separation by 4.1.6.1. We may therefore assume that $e$ is incident with two bag vertices of $T^{\prime}$. Then $e$ is also incident with two bag vertices of $T$, so the $k$-separation $(R, G)$ displayed by the components of $T \backslash e$ is non-sequential by 
(P1). Now $(B, E-B)$ is also a $k$-separation displayed by an edge of $T$, so it follows from Lemma 4.1 .4 that $(B, E-B)$ does not cross $(R, G)$. Thus we may assume, up to switching $R$ and $G$, that $B \subseteq R$ because $B$ is a bag of $T$. Then $(R \cup X, G-X)$ is the partition of $E$ displayed by the components of $T^{\prime} \backslash e$, and $R \cup X$ is $k$-separating by uncrossing $B \cup X$ and $R$, so $(R \cup X, G-X)$ is $\mathcal{T}$-equivalent to $(R, G)$ by Lemma 3.3.10.

4.1.6.3. Every non-sequential $k$-separation of $\lambda$ conforms with $T^{\prime}$.

Subproof. Seeking a contradiction, suppose that $(R, G)$ is a non-sequential $k$-separation of $\lambda$ that does not conform with $T^{\prime}$. Then $(R, G)$ conforms with $T$ because $T$ is a partial $k$-tree, so, by possibly replacing $(R, G)$ by a $\mathcal{T}$-equivalent $k$-separation, we may assume that either $(R, G)$ is displayed by $T$ or $R$ is contained in a bag of $T$. If $(R, G)$ is displayed by $T$, then it follows immediately from 4.1.6.1 and 4.1.6.2 that there is some $k$-separation equivalent to $(R, G)$ that is displayed by $T^{\prime}$. Thus we may assume that $R \subseteq B^{\prime}$ for some bag $B^{\prime} \neq B$ of $T$. We may further assume that both $R \cap\left(B^{\prime}-X\right)$ and $R \cap X$ are non-empty, since $B^{\prime}-X$ is a bag of $T^{\prime}$. We now show that $(R, G)$ is $\mathcal{T}$-equivalent to the $k$-separation $(R-X, G \cup X)$. Since $B \subseteq G$, the set $G \cup X$ is $k$-separating by uncrossing $B \cup X$ and $G$, so, by Lemma 3.3.10, the $k$-separation $(R-X, G \cup X)$ is equivalent to $(R, G)$. But $R-X \subseteq B^{\prime}-X$, so $(R, G)$ conforms with $T^{\prime}$; a contradiction.

It follows from 4.1.6.1, 4.1.6.2, and 4.1.6.3 that $T^{\prime}$ is a partial $k$-tree for $\mathcal{T}$. Moreover, it follows from 4.1.6.1 and 4.1.6.2 that $T$ and $T^{\prime}$ are equivalent partial $k$-trees.

Corollary 4.1.7. Let $T$ be a partial $k$-tree for $\mathcal{T}$, and let $B$ be a nonsequential terminal bag of $T$. If $\left(X_{i}\right)_{i=1}^{m}$ is a partial $k$-sequence for $B$, then there is a $k$-tree $T^{\prime}$ equivalent to $T$ such that $B \cup\left(\bigcup_{i=1}^{m} X_{i}\right)$ is a terminal bag of $T^{\prime}$.

Proof. Assume that $\left(X_{i}\right)_{i=1}^{m}$ is a partial $k$-sequence for $B$. We proceed by induction on $m$. If $m=1$, then there is a partial $k$-tree $T_{1}$ equivalent to 
$T$ with non-sequential terminal bag $B \cup X_{1}$ by Lemma 4.1.6. Thus we may assume that $m \geq 2$, and that the result holds for $m-1$. Then $\left(X_{i}\right)_{i=1}^{m-1}$ is a partial $k$-sequence for $B$, so by induction there is a partial $k$-tree $T_{m-1}$ equivalent to $T$ with non-sequential terminal bag $B \cup\left(\bigcup_{i=1}^{m-1} X_{i}\right)$. Then $X_{m}$ is a non-empty $\mathcal{T}$-weak subset of $E-\left(B \cup\left(\bigcup_{i=1}^{m-1} X_{i}\right)\right)$ such that $B \cup\left(\bigcup_{i=1}^{m} X_{i}\right)$ is $k$-separating, so, by Lemma 4.1.6, there is a partial $k$-tree $T_{m}$ equivalent to $T_{m-1}$, and hence equivalent to $T$, such that $B \cup\left(\bigcup_{i=1}^{m} X_{i}\right)$ is a non-sequential terminal-bag of $T_{m}$, as required.

We have seen that if a partial $k$-tree $T$ has a non-sequential terminal bag $B$, then there is a partial $k$-tree equivalent to $T$ that has terminal bag $B \cup\left(\bigcup_{i=1}^{m} X_{i}\right)$ for any partial $k$-sequence $\left(X_{i}\right)_{i=1}^{m}$ for $B$. We next show that if $\left(X_{i}\right)_{i=1}^{m}$ is a partial $k$-sequence for $E-B$, then there is a partial $k$-tree equivalent to $T$ that has terminal bag $B-\left(\bigcup_{i=1}^{m} X_{i}\right)$.

Lemma 4.1.8. Let $T$ be a partial $k$-tree for $\mathcal{T}$, and let $B$ be a non-sequential terminal bag of $T$. If $X \subseteq B$ is a non-empty $\mathcal{T}$-weak set such that $B-X$ is $k$-separating, then there is a partial $k$-tree $T^{\prime}$ equivalent to $T$ such that $B-X$ is a non-sequential terminal bag of $T^{\prime}$.

Proof. Assume that $X \subseteq B$ is a non-empty $\mathcal{T}$-weak set such that $B-X$ is $k$-separating. Let $u$ be the bag vertex of $T$ that is labelled by $B$. We modify $T$ to produce a $\pi$-labelled tree $T^{\prime}$ by adding a new vertex $v$ adjacent to $u$, relabelling the vertex $u$ by the bag $X$, and labelling $v$ by $B-X$. Then $B-X$ is a terminal bag of $T^{\prime}$, and $B-X$ is non-sequential by Lemma 3.3.10. It is easily verified that $T^{\prime}$ satisfies the first four partial $k$-tree axioms, (P1)(P4). Assume that $T^{\prime}$ does not satisfy the axiom (P5). Then there is a non-sequential $k$-separation $(R, G)$ that does not conform with $T^{\prime}$. Since $T$ is a partial $k$-tree and $T^{\prime}$ only differs from $T$ by adding $v$ and changing the bag $B$, we may assume, by possibly replacing $(R, G)$ by a $\mathcal{T}$-equivalent $k$ separation, that $R \subseteq B$ and that both $R \cap X$ and $R \cap(B-X)$ are non-empty. Now, the set $G \cup X$ is the union of the $k$-separating sets $(E-B) \cup X$ and $G$. Since $((E-B) \cup X) \cap G$ contains $E-B$, and $E-(((E-B) \cup X) \cap G)$ contains 
$R$, the partition $(((E-B) \cup X) \cap G, E-(((E-B) \cup X) \cap G))$ is $\mathcal{T}$-strong, so $\lambda(((E-B) \cup X) \cap G) \geq k$ by (T2). Thus $G \cup X$ is $k$-separating by uncrossing $(E-B) \cup X$ and $G$, and so $(R, G)$ is equivalent to $(R-X, G \cup X)$ by Lemma 3.3.10. But $R-X \subseteq B-X$, so $(R, G)$ conforms with $T^{\prime}$; a contradiction. Thus $T^{\prime}$ is indeed a partial $k$-tree. We now show that $T$ and $T^{\prime}$ are equivalent partial $k$-trees. It is clear that $T \preccurlyeq T^{\prime}$. On the other hand, with the exception of $(B-X, E-(B-X))$, every non-sequential $k$-separation displayed by $T^{\prime}$ is also displayed by $T$. But $(B-X, E-(B-X))$ is $\mathcal{T}$-equivalent to $(B, E-B)$ by Lemma 3.3.10, and $(B, E-B)$ is displayed by $T$. Thus we also have $T^{\prime} \preccurlyeq T$, so $T$ and $T^{\prime}$ are indeed equivalent partial $k$-trees for $\mathcal{T}$.

Corollary 4.1.9. Let $T$ be a partial $k$-tree for $\mathcal{T}$, and let $B$ be a nonsequential terminal bag of $T$. If $\left(X_{i}\right)_{i=1}^{m}$ is a partial $k$-sequence for $E-B$, then there is a partial $k$-tree $T^{\prime}$ equivalent to $T$ with non-sequential terminal bag $B-\left(\bigcup_{i=1}^{m} X_{i}\right)$.

Proof. Assume that $\left(X_{i}\right)_{i=1}^{m}$ is a partial $k$-sequence for $E-B$. We proceed by induction on $m$. If $m=1$, then it follows from Lemma 4.1.8 that there is a partial $k$-tree $T_{1}$ for $\mathcal{T}$ that is equivalent to $T$ with non-sequential terminal bag $B-X_{1}$. Thus we may assume that $m \geq 2$, and that the result holds for $m-1$. Then $\left(X_{i}\right)_{i=1}^{m-1}$ is a partial $k$-sequence for $E-B$, so by induction there is a partial $k$-tree $T_{m-1}$ for $\mathcal{T}$ that is equivalent to $T$ with non-sequential terminal bag $B-\left(\bigcup_{i=1}^{m-1} X_{i}\right)$. Then $X_{m}$ is a non-empty $\mathcal{T}$-weak subset of $B-\left(\bigcup_{i=1}^{m-1} X_{i}\right)$ such that $B-\left(\bigcup_{i=1}^{m} X_{i}\right)$ is $k$-separating, so, by Lemma 4.1 .8 . there is a partial $k$-tree $T_{m}$ for $\mathcal{T}$ that is equivalent to $T_{m-1}$, and hence equivalent to $T$, such that $B-\left(\bigcup_{i=1}^{m} X_{i}\right)$ is a non-sequential terminal bag of $T_{m}$, as required.

Lemma 4.1.10. Let $T$ be a partial $k$-tree for $\mathcal{T}$, and let $B$ be a non-sequential terminal bag of $T$. If $(C, E-C)$ is a non-sequential $k$-separation such that $\mathrm{fcl}_{\mathcal{T}}(B)=\mathrm{fcl}_{\mathcal{T}}(C)$, then there is a partial $k$-tree $T^{\prime}$ equivalent to $T$ with terminal bag $C$. 
Proof. Assume that $(C, E-C)$ is a $k$-separation such that $\mathrm{fcl}_{\mathcal{T}}(B)=\mathrm{fcl}_{\mathcal{T}}(C)$. Then $(C, E-C)$ is $\mathcal{T}$-equivalent to $(B, E-B)$ by Lemma 3.3.11. Let $\left(X_{i}\right)_{i=1}^{n}$ be a $k$-sequence for $B$, and let $\left(Y_{i}\right)_{i=1}^{m}$ be a $k$-sequence for $C$. Then $\mathrm{fcl}_{\mathcal{T}}(B)=$ $B \cup\left(\bigcup_{i=1}^{n} X_{i}\right)$ and $\mathrm{fcl}_{\mathcal{T}}(C)=C \cup\left(\bigcup_{i=1}^{m} Y_{i}\right)$ by Lemma 3.3.7. By Corollary 4.1.7, there is a partial $k$-tree $T^{\prime}$ for $\mathcal{T}$ that is equivalent to $T$ such that $\mathrm{fcl}_{\mathcal{T}}(B)$ is a non-sequential terminal bag of $T^{\prime}$. Now $\left(Y_{m-i+1}\right)_{i=1}^{m}$ is a partial $k$-sequence for $E-\mathrm{fcl}_{\mathcal{T}}(B)$, so, by Corollary 4.1 .9 , there is a partial $k$-tree $T^{\prime \prime}$ for $\mathcal{T}$ that is equivalent to $T^{\prime}$, and hence equivalent to $T$, with terminal bag $C$, as required.

\subsection{Proof of the main theorem}

We can now prove Lemma 4.1.3, from which Theorem 4.2.1 will easily follow.

Proof of Lemma 4.1.3. Suppose that $(R, G)$ is a non-sequential $k$-separation of $\lambda$ that is not equivalent to any $k$-separation displayed by $T$. Then $(R, G)$ conforms with $T$, so we may assume, by possibly replacing $(R, G)$ by an equivalent $k$-separation, that $R$ is properly contained in a bag $B$ of $T$. Let $u$ be the vertex of $T$ labelled by $B$. We distinguish two cases:

(I) $u$ is a leaf of $T$; and

(II) $u$ is not a leaf of $T$.

Consider case (I).

4.2.0.1. $(B, E-B)$ is a non-sequential $k$-separation of $\lambda$.

Subproof. If $u$ is adjacent to a bag vertex, then the result follows immediately from (P1). Assume that $u$ is adjacent to a $k$-flower vertex $v$, and let $\Phi=$ $\left(P_{1}, \ldots, P_{n}\right)$ be the $k$-flower corresponding to $v$. Then $B$ is contained in a petal of $\Phi$ while $E-B$ is contained in $G$, so it follows from Lemma 3.4.5 and Lemma 3.3 .3 that $(B, E-B)$ is non-sequential. 
Now by Corollary 4.1 .9 and Lemma 3.3.15, we may assume, by possibly replacing $T$ by an equivalent partial $k$-tree and replacing $(R, G)$ by an equivalent $k$-separation, that $E-B$ is fully closed with respect to $\mathcal{T}$. Let $Z$ be a $k$-separating set that is maximal with respect to the property that $R \subseteq Z \subsetneq B$. Let $(W, Z)=(E-Z, Z)$.

4.2.0.2. $(W, Z)$ is a non-sequential $k$-separation that is not equivalent to any $k$-separation displayed by $T$.

Subproof. Since $W \subseteq G$ and $Z \subsetneq B$, it follows from Lemma 3.3.3 that $(W, Z)$ is a non-sequential $k$-separation. Now, seeking a contradiction, suppose that $(W, Z)$ is equivalent to a $k$-separation $\left(W^{\prime}, Z^{\prime}\right)$ that is displayed by $T$, where $\mathrm{fcl}_{\mathcal{T}}(Z)=\mathrm{fcl}_{\mathcal{T}}\left(Z^{\prime}\right)$. Since $\left(W^{\prime}, Z^{\prime}\right)$ is non-sequential, and $\mathrm{fcl}_{\mathcal{T}}(Z)=\mathrm{fcl}_{\mathcal{T}}\left(Z^{\prime}\right)$, it follows that $Z^{\prime}$ meets $Z$, and so $Z^{\prime}$ meets $B$. But $Z^{\prime}$ is a union of bags of $T$, so $B$ is contained in $Z^{\prime}$. Thus $\operatorname{fcl}_{\mathcal{T}}(Z)=\mathrm{fcl}_{\mathcal{T}}(B)$ by Lemma 3.3.3. By Lemma 3.3.11, we also have $\operatorname{fcl}_{\mathcal{T}}(W)=\mathrm{fcl}_{\mathcal{T}}(E-B)$. But $E-B$ is fully closed, so it follows that $W \subseteq E-B$; a contradiction because $E-B \subsetneq W$ by the choice of $(W, Z)$.

We note that, by 4.2.0.2, the set $B \cap W$ is $\mathcal{T}$-strong, so the partition $(B \cap W, E-(B \cap W))$ is $\mathcal{T}$-strong. Hence $\lambda(B \cap W) \geq k$ by (T2).

4.2.0.3. If $B \cap W$ is not $k$-separating, then there is a partial $k$-tree $T^{\prime}$ such that $T^{\prime} \succcurlyeq T$ and $T^{\prime}$ displays $(W, Z)$.

Subproof. Assume that $B \cap W$ is not $k$-separating. Let $T^{\prime}$ be the tree that is obtained from $T$ by adjoining a new leaf $v$ adjacent to $u$ such that $v$ is a bag vertex labelled by $Z$, and $u$ is relabelled by $B \cap W$. It is easily verified that $T^{\prime}$ satisfies the first four partial $k$-tree axioms, (P1)-(P4). Assume that it does not satisfy (P5). Then there is a non-sequential $k$-separation $(X, Y)$ that does not conform with $T^{\prime}$. Since $(X, Y)$ conforms with the partial $k$ tree $T$, and $T^{\prime}$ only differs from $T$ by adding $v$ and changing the bag $B$, we may assume, by possibly replacing $(X, Y)$ by a $\mathcal{T}$-equivalent $k$-separation, that $X \subsetneq B$ and that both $X \cap Z$ and $B \cap W \cap X$ are non-empty. Assume 
first that $\lambda(X \cap Z)<k$. Since $E-(X \cap Z)$ is $\mathcal{T}$-strong, it follows that $X \cap Z$ is a member of $\mathcal{T}$ by (T2). Then $(Z-X, E-(Z-X))$ is $\mathcal{T}$-strong, since $(Z, E-Z)$ is non-sequential, so $\lambda(Z-X) \geq k$ by (T2). Now by uncrossing $Y$ and $Z$, whose intersection is $Z-X$, we see that $Y \cup Z$ is $k$ separating. Thus $(X, Y)$ is $\mathcal{T}$-equivalent to $(X-Z, Y \cup Z)$ by Lemma 3.3.10 But $(X-Z, Y \cup Z)$ conforms with $T^{\prime}$; a contradiction. Thus we may now assume that $\lambda(X \cap Z) \geq k$. Then $X \cup Z$ is $k$-separating by uncrossing $X$ and $Z$. If $X \cup Z$ is properly contained in $B$, then $X \cup Z$ contradicts our choice of $Z$. Thus we may assume that $X \cup Z=B$, and hence that $B \cap W=W \cap X$. Then $\lambda(W \cap X)=\lambda(B \cap W)>k$. Thus $\lambda(W \cup X)<k$ by uncrossing $X$ and $W$, and $W \cup X$ is $\mathcal{T}$-strong, so its complement $Z-X$ is a member of $\mathcal{T}$ by (T2). It now follows from Lemma 3.3 .10 that $(X, Y)$ is equivalent to $(B, E-B)$. But $(B, E-B)$ is displayed by $T^{\prime}$, so $(X, Y)$ conforms with $T^{\prime}$; a contradiction. It follows from this contradiction that $T^{\prime}$ is indeed a partial $k$-tree. Clearly $T^{\prime} \succcurlyeq T$. Moreover, the non-sequential $k$-separation $(W, Z)$ is displayed $T^{\prime}$ but not $T$.

Thus, by 4.2.0.3, we may now assume that $B \cap W$ is $k$-separating. Then $\Phi=(Z, B \cap W, E-B)$ is a $k$-flower in $\mathcal{T}$. Let $\Phi^{\prime}=\left(P_{1}, P_{2}, \ldots, P_{n}\right)$ be a tight maximal $k$-flower in $\mathcal{T}$ such that $\Phi^{\prime} \succcurlyeq \Phi$. Then $\Phi^{\prime}$ displays a $k$-separation $(C, E-C)$ that is $\mathcal{T}$-equivalent to $(B, E-B)$. Thus we may assume that $\mathrm{fcl}_{\mathcal{T}}(B)=\mathrm{fcl}_{\mathcal{T}}(C)$. We observe that, since $E-B$ is fully closed, the set $B$ is contained in $C$. Hence $Z$ is contained in $C$. We may also assume, up to labels, that $C=P_{1} \cup \cdots \cup P_{j}$ for some $j \in[n-1]$. Now $\Phi^{\prime}$ also displays a $k$-separation $\left(W^{\prime}, Z^{\prime}\right)$ that is $\mathcal{T}$-equivalent to $(W, Z)$. Since $(C, E-C)$ and $\left(W^{\prime}, Z^{\prime}\right)$ are inequivalent non-sequential $k$-separations, and $Z \subseteq C$, we may assume, by Lemma 3.4.9, that $Z^{\prime} \subseteq C$. Thus, both $(C, E-C)$ and $\left(W^{\prime}, Z^{\prime}\right)$ are displayed by the $k$-flower $\Phi^{\prime \prime}=\left(P_{1}, \ldots, P_{j}, P_{j+1} \cup \cdots \cup P_{n}\right)$.

By Lemma 4.1.10 there is a partial $k$-tree $T^{\prime}$ equivalent to $T$ with terminal bag $C$ labelling the vertex $u$. We now let $T^{\prime \prime}$ be the $\pi$-labelled tree that is obtained from $T^{\prime}$ as follows: we first adjoin a new flower vertex $v$ adjacent to $u$; then adjoin bag vertices $v_{1}, \ldots, v_{j}$ adjacent to $v$ labelling these by 
$P_{1}, \ldots, P_{j}$ respectively; label $v$ by $D$ or $A$ according to the type of $\Phi^{\prime \prime}$, and, if necessary, we impose the cyclic order $\left(v v_{1}, \ldots, v v_{j}, v w\right)$ on the edges incident with $v$; finally, we relabel $u$ by $\emptyset$. We claim that $T^{\prime \prime}$ is a partial $k$-tree such that $T^{\prime \prime} \succcurlyeq T$ and that $T^{\prime \prime}$ displays a $k$-separation that is $\mathcal{T}$-equivalent to $(W, Z)$.

It is easily verified that $(\mathrm{P} 1)$ and $(\mathrm{P} 2)$ hold for $T^{\prime \prime}$. It is also clear that the partition of $E$ displayed by $T^{\prime \prime}-v$ is the $k$-flower $\Phi^{\prime \prime}=\left(P_{1}, \ldots, P_{j}, P_{j+1} \cup\right.$ $\left.\cdots \cup P_{n}\right)$, and we have seen that $\Phi^{\prime \prime}$ displays at least two inequivalent nonsequential $k$-separations, so $\Phi^{\prime \prime}$ has order at least 3 . Moreover, it follows from Lemma 3.4 .8 that $\Phi^{\prime \prime}$ has no $\mathcal{T}$-loose petals. Thus it follows that the axioms (P3) and (P4) hold for $T^{\prime \prime}$. Assume that $T^{\prime \prime}$ does not satisfy (P5). Then there is some non-sequential $k$-separation $(X, Y)$ that does not conform with $T^{\prime \prime}$. Since $(X, Y)$ conforms with $T^{\prime}$, and $T^{\prime \prime}$ only differs from $T^{\prime}$ by changing the bag $C$, it follows that, by possibly replacing $(X, Y)$ by an equivalent $k$-separation, we may assume that $X \subseteq C$, and that $X$ is not contained in the bag $P_{i}$ of $T^{\prime \prime}$ for any $i \in[j]$. Because $\Phi^{\prime}$ is a tight maximal flower in the robust tangle $\mathcal{T}$, it follows from Theorem 3.6.1 that $(X, Y)$ conforms with $\Phi^{\prime}$. Thus there is a $k$-separation $\left(X^{\prime}, Y^{\prime}\right)$ equivalent to $(X, Y)$ such that either:

(i) $\left(X^{\prime}, Y^{\prime}\right)$ is displayed by $\Phi^{\prime}$; or

(ii) $X^{\prime}$ or $Y^{\prime}$ is contained in a petal of $\Phi^{\prime}$.

Assume first that (i) holds. Then, by Lemma 3.4 .9 and since $X \subseteq C$, we may assume that $X^{\prime} \subseteq C$. Then $\left(X^{\prime}, Y^{\prime}\right)$ is displayed by $\Phi^{\prime \prime}$, and hence is displayed by $T^{\prime \prime}$; a contradiction. Thus we may assume that (ii) holds, and that $\operatorname{fcl}_{\mathcal{T}}(X)=\operatorname{fcl}_{\mathcal{T}}\left(X^{\prime}\right)$. Suppose that $X^{\prime}$ is contained in a petal of $\Phi^{\prime}$. Then, by Lemma 3.3.13, the set $X \cap X^{\prime}$ is non-empty, and $X \subseteq C$, so $X^{\prime} \subseteq P_{i}$ for some $i \in[j]$. Hence $X^{\prime}$ is contained in a bag of $T^{\prime \prime}$; a contradiction. Assume then that $Y^{\prime}$ is contained in a petal $P_{i}$ of $\Phi$. If $i \in[j]$, then $Y^{\prime}$ is contained in a bag of $T^{\prime \prime}$. Assume $i \in[j+1, n]$. Then $X \subseteq C \subseteq X^{\prime}$, so $(C, E-C)$ is a $k$-separation that is $\mathcal{T}$-equivalent to $\left(X^{\prime}, Y^{\prime}\right)$ by Lemma 3.3 .3 and Lemma 3.3.11. But $(C, E-C)$ is displayed by $T^{\prime \prime}$, so $(X, Y)$ conforms with $T^{\prime \prime}$; a 
contradiction. Thus every non-sequential $k$-separation of $\lambda$ conforms with $T^{\prime \prime}$. Therefore $T^{\prime \prime}$ is indeed a partial $k$-tree. Clearly $T^{\prime \prime} \succcurlyeq T^{\prime}$, so $T^{\prime \prime} \succcurlyeq T$. Moreover, $T^{\prime \prime}$ displays a $k$-separation that is equivalent to $(W, Z)$. Therefore the lemma holds for case (I).

Consider case (II). Choose a $k$-separating set $Z$ that is maximal with respect to the property that $R \subseteq Z \subseteq B$. Clearly $(Z, E-Z)$ is a nonsequential $k$-separation. Let $T^{\prime}$ be the $\pi$-labelled tree obtained from $T$ by adjoining a new leaf $v$ adjacent to $u$ such that $v$ is a bag vertex labelled by $Z$, and $u$ is relabelled by $B-Z$.

4.2.0.4. $T^{\prime}$ is a partial $k$-tree for $\mathcal{T}$, and $T \preccurlyeq T^{\prime}$.

Subproof. The first four partial $k$-tree axioms, (P1)-(P4), hold immediately for $T^{\prime}$. Assume that (P5) does not hold for $T^{\prime}$. Then there is a non-sequential $k$-separation $(Y, E-Y)$ that does not conform with $T^{\prime}$. Since $(Y, E-Y)$ conforms with $T$, and $T^{\prime}$ only differs from $T$ by adding $v$ and changing the bag $B$, we may assume, by possibly replacing $(Y, E-Y)$ by a $\mathcal{T}$-equivalent $k$-separation, that $Y \subseteq B$ and that both $Y \cap Z$ and $Y \cap(B-Z)$ are nonempty. Assume that $Y \cap Z$ is $\mathcal{T}$-weak. It follows that $\lambda(Z-Y) \geq k$, since the $k$-separation $(Z, E-Z)$ is non-sequential. But $Z-Y=E-(Y \cup(E-Z))$, so $Y \cap(E-Z)=Y-Z$ is $k$-separating by uncrossing the $k$-separating sets $Y$ and $E-Z$. Then, by Lemma 3.3.10, the $k$-separation $(Y, E-Y)$ is equivalent to $(Y-Z, E-(Y-Z))$. But $(Y-Z, E-(Y-Z))$ conforms with $T^{\prime}$; a contradiction. Thus we may assume that $Y \cap Z$ is $\mathcal{T}$-strong. Then $(Y \cap Z, E-(Y \cap Z))$ is $\mathcal{T}$-strong, so $\lambda(Y \cap Z) \geq k$ by (T2). Then $Y \cup Z$ is $k$-separating by uncrossing $Y$ and $Z$; a contradiction of the maximality of $Z$. Thus $T^{\prime}$ satisfies (P5), so $T^{\prime}$ is a indeed a partial $k$-tree, and it is clear that $T \preccurlyeq T^{\prime}$.

Now $Z$ labels a leaf of $T^{\prime}$, and $R \subseteq Z$, so it follows by case (I) that there is a partial $k$-tree $T^{\prime \prime} \succcurlyeq T^{\prime}$ such that $T^{\prime \prime}$ displays a non-sequential $k$-separation that is not equivalent to any $k$-separation displayed by $T^{\prime}$. Hence $T^{\prime \prime} \succcurlyeq T$ 
and $T^{\prime \prime}$ displays a non-sequential $k$-separation that is not equivalent to any $k$-separation displayed by $T$.

The following is the main theorem of this thesis.

Theorem 4.2.1. Let $\mathcal{T}$ be a robust tangle of order $k$ in a connectivity system $(E, \lambda)$. If $T$ is a maximal partial $k$-tree for $\mathcal{T}$, then every non-sequential $k$ separation of $\lambda$ is equivalent to some $k$-separation displayed by $T$.

Proof. Assume that $T$ is a maximal partial $k$-tree for $\mathcal{T}$. If there are no nonsequential $k$-separations of $\lambda$, then the theorem holds. Suppose that $(R, G)$ is a non-sequential $k$-separation of $\lambda$. Then, by Lemma 4.1.2, there is a tight maximal $k$-flower in $\mathcal{T}$ that displays a $k$-separation equivalent to $(R, G)$, and so, by Corollary 4.1.1, there is a partial $k$-tree $T^{\prime}$ for $\mathcal{T}$ that displays a nonsequential $k$-separation equivalent to $(R, G)$. Thus we may assume that $T$ is a non-trivial partial $k$-tree for $\mathcal{T}$. Then the theorem holds, or else, by Lemma 4.1.3, we contradict the maximality of $T$. 


\section{Chapter 5}

\section{Applications}

In this chapter, we apply the results obtained in Chapter 3 and Chapter 4 to $k$-connected and vertically $k$-connected matroids.

\subsection{Tangles in $k$-connected matroids}

Let $k \geq 3$, and let $M$ be a $k$-connected matroid on ground set $E$. Recall that a tangle $\mathcal{T}$ of order $k$ in $M$ is a tangle of order $k$ in the connectivity system $\left(E, \lambda_{M}\right)$. The next lemma was first proved by Dharmatilake [6]; for completeness, we prove it here.

Lemma 5.1.1. Let $k \geq 3$, and let $M$ be a $k$-connected matroid having at least $3(k-2)+1$ elements. Then $\mathcal{T}=\{X \subseteq E(M)|| X \mid \leq k-2\}$ is the unique tangle of order $k$ in $M$.

Proof. We first show that $\mathcal{T}=\{X \subseteq E(M)|| X \mid \leq k-2\}$ is a tangle of order $k$ in $M$. Suppose that $X \subseteq E(M)$ and $|X| \leq k-2$. Then $\lambda_{M}(X) \leq$ $r_{M}(X)+1 \leq k-1$, so (T1) holds. Suppose that $(X, Y)$ is a $j$-separation for some $j \in[k-1]$. Since $M$ is $k$-connected, up to switching $X$ and $Y$, we can assume that $|X|<j \leq k-1$, so $X \in \mathcal{T}$. Thus (T2) holds. The union of any three subsets of size less than $k-2$ has at most $3(k-2)$ elements, 
which is less than $|E(M)|$, so (T3) holds. Finally, for each $e \in E(M)$, $|E(M)-e| \geq 3(k-2)>k-2$, so $E(M)-e \notin \mathcal{T}$.

Now suppose that $\mathcal{T}^{\prime}$ is a tangle of order $k$ in $M$. Suppose that there is some $X \in \mathcal{T}^{\prime}$ such that $|X| \geq k-1$. We may assume that $X$ is a maximal member of $\mathcal{T}^{\prime}$. Then $|E(M)-X|<k-1$ because $M$ is $k$-connected. Moreover, $E(M)-X$ is non-empty by (T3), so there is some $e \in E(M)-X$. Then $\lambda(E(M)-(X \cup e))<k$, and $X \cup e \notin \mathcal{T}^{\prime}$ because $X$ is maximal. Thus $E(M)-(X \cup e) \in \mathcal{T}^{\prime}$ by $(\mathrm{T} 2)$. But $e, E-(X \cup e), X \in \mathcal{T}^{\prime}$ and their union is $E(M)$, contradicting (T3). Therefore every member of $\mathcal{T}^{\prime}$ has $k-2$ or fewer elements, so it follows from (T2) that $\mathcal{T}^{\prime}=\mathcal{T}$.

Let $M$ be a $k$-connected matroid on $E$ with at least $3(k-2)+1$ elements. Then $M$ has a unique tangle of order $k$ by Lemma 5.1.1. For convenience, we translate some of the terminology we have developed to this context.

A subset $X$ of $M$ is $\mathcal{T}$-strong if $|X| \geq k-1$; otherwise $X$ is $\mathcal{T}$-weak. A $k$-separation $(X, Y)$ of $M$ is $\mathcal{T}$-strong if both $X$ and $Y$ are $\mathcal{T}$-strong sets, that is, if $|X|,|Y| \geq k-1$. A $\mathcal{T}$-strong $k$-separating set $X$ is fully closed if $X \cup Y$ is not $k$-separating for all non-empty $Y \subseteq E-X$ such that $|Y| \leq k-2$. The full closure of a $\mathcal{T}$-strong $k$-separating set $X$, denoted $\mathrm{fcl}_{\mathcal{T}}(X)$, is the intersection of all fully-closed $k$-separating sets that contain $X$. It follows from Corollary 3.3.2 that $\operatorname{fcl}_{\mathcal{T}}(X)$ is a fully-closed $k$-separating set. By Lemma 3.3.7, we can realise $\mathrm{fcl}_{\mathcal{T}}(X)$ as $X \cup\left(\bigcup_{i=1}^{m} X_{i}\right)$, where $\left(X_{i}\right)_{i=1}^{m}$ is a maximal sequence of disjoint subsets of $E-X$ such that $\left|X_{i}\right| \leq k-2$ and $X \cup\left(\cup_{i=1}^{j} X_{i}\right)$ is $k$-separating for each $j \in[m]$. Let $X$ be a $\mathcal{T}$-strong $k$-separating set. Then $E-X$ is sequential if $\mathrm{fcl}_{\mathcal{T}}(X)=E$.

Two $\mathcal{T}$-strong $k$-separations $(A, B)$ and $(X, Y)$ of $M$ are equivalent if $\left\{\operatorname{fcl}_{\mathcal{T}}(A), \mathrm{fcl}_{\mathcal{T}}(B)\right\}=\left\{\mathrm{fcl}_{\mathcal{T}}(X), \mathrm{fcl}_{\mathcal{T}}(Y)\right\}$. In other words, $(A, B)$ and $(X, Y)$ are equivalent if we can obtain $(X, Y)$ from $(A, B)$ by moving some sequence of non-empty subsets, each having $k-2$ or fewer elements, across the partition in such a way that, at any step, the result is a $k$-separation of $M$. A $\mathcal{T}$-strong $k$-separation $(X, Y)$ is sequential if either $X$ or $Y$ is sequential.

We note that for $k=3$, the notions of equivalent and sequential 3- 
separations here are precisely those of Oxley, Semple, and Whittle in [23]. Similarly, for $k=4$, the notion of equivalent and sequential 4-separations here are precisely those developed by Aikin [1].

A partition $\left(P_{1}, \ldots, P_{n}\right)$ of $E$ is a $k$-flower in $M$ with petals $P_{1}, \ldots, P_{n}$ if, for all $i,\left|P_{i}\right| \geq k-1$, and $P_{i}$ and $P_{i} \cup P_{i+1}$ are $k$-separating, where all subscripts are interpreted modulo $n$. We note that, for $k=3$, a 3 -flower in $M$ is a flower in $M$ as defined in 23 .

Corollary 5.1.2. Let $k \geq 3$, and let $M$ be a $k$-connected matroid having at least $8(k-2)+1$ elements. If $\mathcal{T}$ is a tangle of order $k$ in $M$, then $\mathcal{T}$ is robust.

Proof. By Lemma 5.1.1, $\mathcal{T}=\{X \subseteq E(M)|| X \mid \leq k-2\}$. Thus no eight members of $\mathcal{T}$ can cover $E(M)$.

Thus every $k$-connected matroid with at least $8(k-2)+1$ elements has a unique robust tangle of order $k$. The next result follows immediately from Theorem 3.6.1 and Corollary 5.1.2

Corollary 5.1.3. Let $k \geq 3$, and let $M$ be a $k$-connected matroid with at least $8(k-2)+1$ elements. Let $\Phi$ be a tight maximal $k$-flower in $M$. Then every non-sequential $k$-separation of $M$ conforms with $\Phi$.

A partial $k$-tree for $M$ is a partial $k$-tree for the unique tangle in $M$, which we defined in Chapter 4. We note that for $k=3$, the partial $k$-tree we defined in Chapter 4 is also a partial 3-tree as defined in [23].

We have the following immediate corollary of Theorem 4.2.1 and Corollary 5.1 .2

Corollary 5.1.4. Let $k \geq 3$, and let $M$ be a $k$-connected matroid with at least $8(k-2)+1$ elements. If $T$ is a maximal partial $k$-tree for $M$, then every non-sequential $k$-separation of $M$ is equivalent to a $k$-separation displayed by $T$.

We note that the case $k=3$ in Corollary 5.1 .4 was proved by Oxley, Semple, and Whittle [23]. The case $k=4$ was proved by Aikin and Oxley (private communication). 


\subsection{Vertically $k$-connnected matroids}

First, we need the following lemma giving equivalent conditions to (T3).

Lemma 5.2.1. [9, Lemma 3.1] Let $\lambda$ be a connectivity function on $E$, and let $\mathcal{T}$ be a collection of subsets of $E$ that satisfies (T1), (T2), (T4), and:

(T3a) If $A \subseteq B, B \in \mathcal{T}$, and $\lambda(A)<k$, then $A \in \mathcal{T}$.

(T3b) If $(A, B, C)$ is a partition of $E$, then $\mathcal{T}$ cannot contain all three of $A$, $B$, and $C$.

Then $\mathcal{T}$ is a tangle of order $k$ in $\lambda$.

We now show that vertically $k$-connected matroids of sufficiently large rank have a unique tangle of order $k$.

Lemma 5.2.2. Let $k \geq 3$. Let $M$ be a vertically $k$-connected matroid of rank at least $3(k-2)+1$, and let $r$ be the rank function of $M$. Then $\mathcal{T}=\{X \subseteq$ $E(M) \mid r(X) \leq k-2\}$ is the unique tangle of order $k$ in $M$.

Proof. We first show that $\mathcal{T}=\{X \subseteq E(M) \mid r(X) \leq k-2\}$ is a tangle of order $k$ in $M$. Suppose that $X \subseteq E(M)$ and $r(X) \leq k-2$. Then $\lambda(X) \leq r(X)+1 \leq k-1$, so (T1) holds. Let $(X, Y)$ be a separation of order $j$ for some $j<k$. Since $M$ is vertically $k$-connected, up to switching $X$ and $Y$, we can assume that $r(X)<j \leq k-1$, so $X \in \mathcal{T}$. Thus (T2) holds. Suppose that $A \subseteq B, B \in \mathcal{T}$, and $\lambda(A)<k$. Then $r(A) \leq r(B) \leq k-2$, so $A \in \mathcal{T}$. Thus $\mathcal{T}$ satisfies (T3a). Suppose that $(A, B, C)$ is a partition of $E(M)$. Then $r(A)+r(B)+r(C) \geq 3(k-2)+1$, by submodularity, so at least one of $A, B$, and $C$ has rank at least $k-1$. Hence $\mathcal{T}$ cannot contain all three of $A, B$, and $C$, and so (T3b) holds. Finally, for each $e \in E(M)$, $r(E(M)-e) \geq 3(k-2)>k-2$, so $E(M)-e \notin \mathcal{T}$. Thus (T4) holds.

Now suppose that $\mathcal{T}^{\prime}$ is a tangle of order $k$ in $M$. Suppose that there is some $X \in \mathcal{T}^{\prime}$ and $r(X) \geq k-1$. We may assume that $X$ is a maximal member of $\mathcal{T}^{\prime}$. Then $r(E(M)-X) \leq k-2$ because $M$ is vertically $k$-connected. Let 
$e \in E(M)-X$. Then $\lambda(E(M)-(X \cup e))<k$, and $X \cup e \notin \mathcal{T}^{\prime}$ because $X$ is maximal. Thus $E(M)-(X \cup e) \in \mathcal{T}^{\prime}$ by (T2). Then $e, E-(X \cup e), X \in \mathcal{T}^{\prime}$ and their union is $E(M)$, contradicting (T3). Therefore every member of $\mathcal{T}^{\prime}$ has rank at most $k-2$, so $\mathcal{T}^{\prime} \subseteq \mathcal{T}$. It now follows from (T2) and the vertical $k$-connectivity of $M$ that $\mathcal{T}^{\prime}=\mathcal{T}$.

Let $M$ be a vertically $k$-connected matroid on $E$ with rank at least $3(k-$ $2)+1$, and let $r$ be the rank function of $M$. Then $M$ has a unique tangle of order $k$ by Lemma 5.2.2. For convenience, we translate some of the theory we have developed to this context.

A subset $X$ of $M$ is $\mathcal{T}$-strong if $r(X) \geq k-1$; otherwise $X$ is $\mathcal{T}$-weak. A $k$-separation $(X, Y)$ of $M$ is $\mathcal{T}$-strong if both $X$ and $Y$ are $\mathcal{T}$-strong sets, that is, if $r(X), r(Y) \geq k-1$. A $\mathcal{T}$-strong $k$-separating set $X$ is fully closed if $X \cup Y$ is not $k$-separating for all non-empty $Y \subseteq E-X$ such that $r(Y) \leq$ $k-2$. The full closure of a $\mathcal{T}$-strong $k$-separating set $X$, denoted $\mathrm{fcl}_{\mathcal{T}}(X)$, is the intersection of all fully-closed $k$-separating sets that contain $X$. It follows from Corollary 3.3 .2 that $\mathrm{fcl}_{\mathcal{T}}(X)$ is a fully-closed $k$-separating set. By Lemma 3.3.7, we can realise $\operatorname{fcl}_{\mathcal{T}}(X)$ as $X \cup\left(\bigcup_{i=1}^{m} X_{i}\right)$, where $\left(X_{i}\right)_{i=1}^{m}$ is a maximal sequence of disjoint subsets of $E-X$ such that $r\left(X_{i}\right) \leq k-2$ and $X \cup\left(\bigcup_{i=1}^{j} X_{i}\right)$ is $k$-separating for each $j \in[m]$. Let $X$ be a $\mathcal{T}$-strong $k$-separating set. Then $E-X$ is sequential if $\operatorname{fcl}_{\mathcal{T}}(X)=E$.

Two $\mathcal{T}$-strong $k$-separations $(A, B)$ and $(X, Y)$ are equivalent if $\left\{\operatorname{fcl}_{\mathcal{T}}(A), \mathrm{fcl}_{\mathcal{T}}(B)\right\}=\left\{\mathrm{fcl}_{\mathcal{T}}(X), \mathrm{fcl}_{\mathcal{T}}(Y)\right\}$. In other words, $(A, B)$ and $(X, Y)$ are equivalent if we can obtain $(X, Y)$ from $(A, B)$ by moving some sequence of non-empty subsets, each having rank $k-2$ or fewer, across the partition in such a way that, at any step, the result is a $k$-separation. A $\mathcal{T}$-strong $k$-separation $(X, Y)$ is sequential if either $X$ or $Y$ is sequential.

A partition $\left(P_{1}, \ldots, P_{n}\right)$ of $E$ is a $k$-flower in $M$ with petals $P_{1}, \ldots, P_{n}$ if, for all $i, r\left(P_{i}\right) \geq k-1$, and $P_{i}$ and $P_{i} \cup P_{i+1}$ are $k$-separating, where all subscripts are interpreted modulo $n$.

Corollary 5.2.3. Let $k \geq 3$, and let $M$ be a vertically $k$-connected matroid 
with rank at least $8(k-2)+1$. If $\mathcal{T}$ is a tangle of order $k$ in $M$, then $\mathcal{T}$ is robust.

Proof. By Lemma 5.2.2, we know that $\mathcal{T}=\{X \subseteq E(M) \mid r(X) \leq k-2\}$. Thus (T1), (T2), and (T4) hold. Moreover, it is straightforward to check that (RT3a) and (RT3b) hold.

Thus every vertically $k$-connected matroid with at least $8(k-2)+1$ elements has a unique robust tangle of order $k$. The next result follows immediately from Corollary 5.2.3 and Theorem 3.6.1.

Corollary 5.2.4. Let $k \geq 3$, and let $M$ be a vertically $k$-connected matroid with rank at least $8(k-2)+1$. If $\Phi$ is a tight maximal $k$-flower in $M$, then every non-sequential $k$-separation of $M$ conforms with $\Phi$.

A partial $k$-tree for $M$ is a partial $k$-tree for the unique tangle of order $k$ in $M$, which we defined in Chapter 4 .

We have the following immediate corollary of Theorem 4.2.1 and Corollary 5.2 .3 .

Corollary 5.2.5. Let $k \geq 3$, and let $M$ be a vertically $k$-connected matroid with rank at least $8(k-2)+1$. If $T$ is a maximal partial $k$-tree for $M$, then every non-sequential $k$-separation of $M$ is equivalent to a $k$-separation displayed by $T$.

We note that, by duality, everything in this section for vertically $k$ connected matroids also holds for cyclically $k$-connected matroids. 


\section{Bibliography}

[1] Aikin, J. The structure of 4-separations in 4-connected matroids. PhD thesis, Louisiana State University, 2009.

[2] Aikin, J., And Oxley, J. The structure of crossing separations in matroids. Advances in Applied Mathematics 41, 1 (2008), 10-26.

[3] Bodlaender, H., And Koster, A. Combinatorial optimization on graphs of bounded treewidth. The Computer Journal 51, 3 (2008), 255269.

[4] Cunningham, W., And Edmonds, J. A combinatorial decomposition theory. Can. J. Math. 32 (1980), 734-765.

[5] Dharmatilake, J. Binary matroids of branch-width 3. PhD thesis, Ohio State University, 1994.

[6] Dharmatilake, J. A min-max theorem using matroid separations. Contemporary Mathematics $19^{77}$ (1996), 333-342.

[7] Geelen, J., Gerards, A., Robertson, N., and Whittle, G. On the excluded minors for the matroids of branch-width $\mathrm{k}$. Journal of Combinatorial Theory, Series B 88, 2 (2003), 261-265.

[8] Geelen, J., Gerards, A., And Whittle, G. Branch-Width and Well-Quasi-Ordering in Matroids and Graphs. Journal of Combinatorial Theory, Series B 84, 2 (2002), 270-290. 
[9] Geelen, J., Gerards, B., Robertson, N., And Whittle, G. Obstructions to branch-decomposition of matroids. Journal of Combinatorial Theory, Series B 96, 4 (2006), 560-570.

[10] Geelen, J., Gerards, B., And Whittle, G. Towards a structure theory for matrices and matroids. In International Congress of Mathematicians (2006), vol. 3, Citeseer, pp. 827-842.

[11] Geelen, J., Gerards, B., And Whittle, G. Excluding a planar graph from GF(q)-representable matroids. Journal of Combinatorial Theory, Series B 97, 6 (2007), 971-998.

[12] Geelen, J., Gerards, B., And Whittle, G. Towards a matroidminor structure theory. Oxford Lecture Series in Mathematics and Its Applications 34 (2007), 72.

[13] Geelen, J., Gerards, B., And Whittle, G. Tangles, treedecompositions and grids in matroids. Journal of Combinatorial Theory, Series B 99, 4 (2009), 657-667.

[14] Geelen, J., And Whittle, G. Inequivalent representations of matroids over prime fields, submitted.

[15] Geelen, J., And Whittle, G. Branch-Width and Rota's Conjecture. Journal of Combinatorial Theory, Series B 86, 2 (2002), 315-330.

[16] Hall, R., Oxley, J., Semple, C., and Whittle, G. On matroids of branch-width three. Journal of Combinatorial Theory, Series B 86, 1 (2002), 148-171.

[17] Hicks, I., AND McMurray JR, N. The branchwidth of graphs and their cycle matroids. Journal of Combinatorial Theory, Series B 97, 5 (2007), 681-692.

[18] HLinĚNÝ, P. On the excluded minors for matroids of branch-width three. The Electronic Journal of Combinatorics 9, R32 (2002), 1. 
[19] Hliněný, P., And Whittle, G. Matroid tree-width. European Journal of Combinatorics 27, 7 (2006), 1117-1128.

[20] Kruskal, J. Well-Quasi-Ordering, The Tree Theorem, and Vazsonyi's Conjecture. Transactions of the American Mathematical Society 95, 2 (1960), 210-225.

[21] Nash-Williams, C. S. J. A. On well-quasi-ordering finite trees. In Mathematical Proceedings of the Cambridge Philosophical Society (1963), vol. 59, Cambridge Univ Press, pp. 833-835.

[22] Oxley, J. Matroid theory. Oxford University Press, USA, 2006.

[23] Oxley, J., Semple, C., And Whittle, G. The structure of the 3separations of 3-connected matroids. Journal of Combinatorial Theory, Series B 92, 2 (2004), 257-293.

[24] Oxley, J., Semple, C., And Whittle, G. The structure of the 3-separations of 3-connected matroids II. European Journal of Combinatorics 28, 4 (2007), 1239-1261.

[25] Robertson, N., And Seymour, P. Graph minors. II. Algorithmic aspects of tree-width. Journal of algorithms 7, 3 (1986), 309-322.

[26] Robertson, N., And Seymour, P. Graph minors. V. Excluding a planar graph. Journal of Combinatorial Theory, Series B 41, 1 (1986), 92-114.

[27] Robertson, N., And Seymour, P. Graph minors. IV. Tree-width and well-quasi-ordering. Journal of Combinatorial Theory, Series B 48 , 2 (1990), 227-254.

[28] Robertson, N., And Seymour, P. Graph minors. X. Obstructions to tree-decomposition. Journal of Combinatorial Theory, Series B 52, 2 (1991), 153-190. 
[29] Robertson, N., And Seymour, P. Graph minors. XVI. Excluding a non-planar graph. Journal of Combinatorial Theory, Series B 89, 1 (2003), 43-76.

[30] Robertson, N., And Seymour, P. Graph minors. XX. Wagner's conjecture. Journal of Combinatorial Theory, Series B 92, 2 (2004), $325-357$.

[31] Tutte, W. Menger's theorem for matroids. J. Res. Nat. Bur. Standards Sect. B 69B (1966), 49-53. 


\section{Index}

2-equivalent, 27

3-tree, 24

almost partial 3-tree, 23

anemone, 19

copaddle, 19

paddle, 19

spike-like, 19

vertex, 24

bag, 22, 59

terminal, 59

vertex, 22, 59

branch-decomposition, 10

width, 10

branch-width

connectivity function, 10

graph, 10

matroid, 10

concatenation, 39

conforms

with a $\pi$-labelled tree, 60

with a $k$-flower in $\mathcal{T}$, 46

with a flower, 22

with an almost partial 3-tree, 24

connectivity function, 5,28 of a graph, 6

of a matroid, 6

connectivity system, 5, 29

cross, 19

petals, 46

cyclically $k$-connected, 8

daisy, 19

swirl-like, 19

Vámos-like, 19

vertex, 24

displayed

by a flower, 21

by an almost partial 3-tree, 24

equivalent

3 -separations in 3-connected matroids, 18

flowers, 21

partial 3-trees, 24

flower, 19

order, 21

petal, 19

vertex, 24

full 2-span, 27

full closure 
in 3-connected matroids, 18 in $\mathcal{T}, 32$

fully closed set

in $\mathcal{T}, 31$

in a 3-connected matroid, 18

$k$-anemone, 26, 39

$k$-connected

graph, 7

matroid, 7

$k$-daisy, 26, 39

$k$-flower

in a connectivity function, 26,38

$k$-flower in $\mathcal{T}, 38$

order, 40

petal, 38

tight, 40

vertex, 60

$k$-separating partition

of a matroid, 7

$k$-separating set

exact, 7,29

in a connectivity function, 7,29

in a matroid, 7

$k$-separation

exact, 7, 29

of a connectivity function, 7,29

of a matroid, 7

laminar, 62

local connectivity, 19,26

loose element, 21

petal, 22

maximal

$k$-flower in $\mathcal{T}, 53$

flower, 22

member of a tangle, 15

partial 3-tree, 24

partial $k$-sequence, 34

partial $k$-trees, 61

tangle, 13

partial 3-tree, 24

partial $k$-sequence, 33

$k$-sequence, 35

partial $k$-tree, 60

trivial, 61

partition, 5

$\Phi$-minimum, 46

$\pi$-labelled tree, 22,59

polymatroid, 6, 26

quasi-order, 21, 39

$(R, G)$-strong, 48

$(R, G)$-weak, 48

refines, 40

robust tangle, 52

sequential

3 -separations in 3-connected matroids, 18

4-separation in 4-connected matroid, 27 
strongly crossed, 46

submodular, 5, 28

symmetric, 5,28

$\mathcal{T}$-equivalent

$k$-flowers, 39

$k$-separating sets, 32

$k$-separations, 35

partial $k$-trees, 61

$\mathcal{T}$-loose petal, 41

$\mathcal{T}$-sequential

$k$-separating set, 35

$k$-separation, 35

$\mathcal{T}$-strong

partition, 30

separation, 30

set, 30

$\mathcal{T}$-weak

partition, 30

separation, 30

set, 30

tangle

distinguishing separation, 14

in a connectivity system, 12,30

induced matroid, 17

tight

element, 21

flower, 22

petal, 22

tree-decomposition, 9

width, 9

tree-width, 9 uncrossing, 29

up to labels, 39

vertical $k$-separation, 8

vertical connectivity, 8

weakly crossed, 46 